\title{
Nicht was Du denkst! Was wir wirklich von Wahlvorhersagen lernen können
}

\author{
Strijbis, Oliver
}

\begin{abstract}
As this forum demonstrates, the competition between election forecasting models along the US model becomes standard also in Germany. This raises the question about the scientific relevance of this undertaking. It is often argued that election forecasts provide a particularly hard and hence good test for the falsification of theories of electoral behavior. In this contribution, I contest this argument and show that the forecast models presented in this issue hardly contribute to the falsification of theories. Instead I refer to an often overlooked relevance of election forecasts consisting in the models ${ }^{6}$ potential for opera-tionalizing important variables. I illustrate my argument by referring to the literature on prediction markets.
\end{abstract}

DOI: https://doi.org/10.5771/0032-3470-2017-3-442

Posted at the Zurich Open Repository and Archive, University of Zurich

ZORA URL: https://doi.org/10.5167/uzh-145765

Journal Article

Originally published at:

Strijbis, Oliver (2017). Nicht was Du denkst! Was wir wirklich von Wahlvorhersagen lernen können. Politische Vierteljahresschrift, 58(3):442-451.

DOI: https://doi.org/10.5771/0032-3470-2017-3-442 


\section{Politische \\ Vierteljahresschrift}

\section{Zeitschrift der Deutschen Vereinigung für Politikwissenschaft}

Gegründet im Auftrag der Vereinigung von Prof. Dr. Karl Dietrich Bracher †, Prof. Dr. Gert von Eynern †, Prof. Dr. Otto Heinrich von der Gablentz †, Prof. Dr. Gerhard Leibholz † und Prof. Dr. Dolf Sternberger †.

Herausgegeben vom Vorstand der Deutschen Vereinigung für Politikwissenschaft

Redaktion: Prof. Dr. Mathias Albert, Bielefeld; Prof. Dr. Michael Haus, Heidelberg; Prof. Dr. Michèle Knodt, Darmstadt (ViSdP); Prof. Dr. Samuel Salzborn, Gießen (PVS Literatur); Prof. Dr. Kai-Uwe Schnapp, Hamburg; Prof. Dr. Annette Elisabeth Töller, Hagen.

\section{BERICHT}

Brian Dietrich, Olga Herzog und Kai-Uwe Schnapp

Karriereverläufe in der Politikwissenschaft. Ergebnisse der Mitgliederstudie 2015 der DVPW ...

\section{ABHANDLUNG}

Sabrina Jasmin Mayer und Martin Schultze

Multiple Parteiidentifikation, politische Involvierung und Cross-Pressures

\section{FORUM}

Thomas Gschwend und Helmut Norpoth

Machtwechsel in Sicht? Die Vorhersage des Kanzlermodells für die Bundestagswahl 2017. ....

Arndt Leininger und Mark A. Kayser

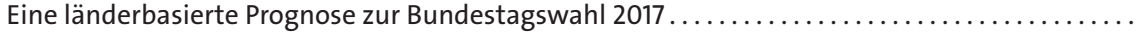

Simon Munzert, Lukas Stötzer, Thomas Gschwend, Marcel Neunhoeffer und Sebastian Sternberg Zweitstimme.org. Ein strukturell-dynamisches Vorhersagemodell für Bundestagswahlen .....

Oliver Strijbis

Nicht was Du denkst! Was wir wirklich von Wahlvorhersagen lernen können

Volker Best

Studierende im Wahl-Werber-Fieber. Die Erstellung von Wahlplakaten zur Bundestagswahl 2017 im „Praxisseminar Wahlkampagne“

\section{PVS-LITERATUR}

\section{BESPRECHUNGEN}

\section{POLITISCHE THEORIE UND IDEENGESCHICHTE}

Fine, Sarah, und Lea Ypi (Hrsg.). Migration in Political Theory. The Ethics of Movement and

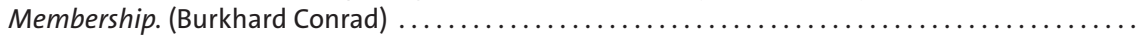

Breuer, Stefan. Kritische Theorie. Schlüsselbegriffe, Kontroversen, Grenzen. (Reinhard Mehring) ..

\section{VERGLEICH POLITISCHER SYSTEME}

von Alemann, Ulrich, Martin Morlok, und Sebastian Roßner (Hrsg.). Politische Parteien in Frankreich und Deutschland. Späte Kinder des Verfassungsstaates. (Sabine Riedel) ... 
Ringdal, Kristen, Sabrina P. Ramet, und Danica Fink-Hafner (eds.). Small States, Big Challenges. Norway and Slovenia in Comparative Perspective. (Hermann Groß) . . .

\section{POLITISCHES SYSTEM DEUTSCHLAND}

Papier, Hans-Jürgen, Ursula Münch, und Gero Kellermann (Hrsg.). Freiheit und Sicherheit. Verfassungspolitik, Grundrechtsschutz, Sicherheitsgesetze. (Markus Löffelmann).

Karlauf, Thomas. Helmut Schmidt. Die späten Jahre.

Spohr, Kristina. Helmut Schmidt. Der Weltkanzler. (Helga Haftendorn).

\section{INTERNATIONALE BEZIEHUNGEN}

Börzel, Tanja A. und Thomas Risse (Hrsg.). The Oxford Handbook of Comparative Regionalism. (Ludger Kühnhardt)

Adomeit, Hannes. Imperial Overstretch: Germany in Soviet Policy from Stalin to Gorbachev. An Analysis based on New Archival Evidence, Memoirs, and Interviews. (Helga Haftendorn)......... 490

Deitelhoff, Nicole, und Michael Zürn. Lehrbuch der Internationalen Beziehungen: Per Anhalter durch die IB-Galaxis. (Ralph Rotte).

\section{EUROPÄISCHE INTEGRATION}

Fruhstorfer, Anna, und Michael Hein (Hrsg.). Constitutional Politics in Central and Eastern Europe. From Post-Socialist Transition to the Reform of Political Systems. (Sebastian Wolf)

\section{POLITIK UND GESCHICHTE}

Senfft, Alexandra. Der lange Schatten der Täter. Nachkommen stellen sich ihrer NS-Familiengeschichte. (Matthias Falter).

Hochgeschwender, Michael. Die Amerikanische Revolution. Geburt einer Nation, 1763-1815.

(Sebastian Huhnholz).

Autorinnen und Autoren der Buchbesprechungen 501 


\title{
Machtwechsel in Sicht? Die Vorhersage des Kanzlermodells für die Bundestagswahl 2017
}

\author{
Thomas Gschwend und Helmut Norpoth
}

\begin{abstract}
A Change of the Guard? The Forecast of the Chancellor Model for 2017
Abstract: The Chancellor Model predicts that the 2017 Bundestag election will most likely confirm Angela Merkel for another term as head of the federal government. As of June this year, she leads her Social Democratic challenger Martin Schulz by a wide enough margin in chancellor popularity to overcome any fatigue with her tenure after three terms. Besides continuing with the Grand Coalition, Merkel also has the option of entering a Jamaicacoalition (with the FDP and Greens), which would command a majority in the new Bundestag. A shift in chancellor popularity, however, would change the forecast. In the event that Schulz ultimately surpasses Merkel in chancellor popularity, as he did in late February, the SPD candidate would be more likely to head the next German government.
\end{abstract}

Keywords: Chancellor Model, Forecast, German Federal Election 2017, Coalition, Cost of Ruling

Stichworte: Kanzlermodell, Vorhersage, Bundestagswahl 2017, Koalition, Abnützungseffekt

\section{Einleitung ${ }^{1}$}

Wenn die Stimmen am Wahlabend des 24. September 2017 ausgezählt sind, wird die Bundesrepublik höchstwahrscheinlich wieder Angela Merkel als Regierungschefin bekommen. Dies ist die Prognose des Kanzlermodells (Gschwend u. Norpoth 2001), basierend auf den Informationen, die uns bis Anfang Juni 2017 vorliegen und bis zu vier Wochen vor der Wahl noch aktualisiert werden. Erst zu diesem Zeitpunkt kann es nach der Logik des Kanzlermodells eine endgültige Prognose geben. Ein Machtwechsel liegt nicht in der Luft. Dazu ist Martin Schulz, der sozialdemokratische Kanzlerkandidat, derzeit zu weit weg vom „Mr.100 Prozent", wie er gerne in den Medien bezeichnet wird. Mit seinem derzeitigen Popularitätswert schickt er sich nicht an, die Kanzlerschaft der christdemokratischen Amtsinhaberin Angela Merkel und ihre 12-jährige Amtszeit als Bundeskanzlerin beenden zu können.

Diese Einsicht verdanken wir dem Kanzlermodell, das sich bereits bei den letzten vier Bundestagswahlen bewährt hat. Unsere theoretischen Ansätze zur Erklä-

1 Wir danken den beiden anonymen Gutachtern sowie Marc Debus für wertvolle Hinweise und Christel Selzer für ihre redaktionelle Arbeit am Manuskript. 
rung von Wahlverhalten und dem daraus resultierenden Zusammenwirken von lang-, mittel- und kurzfristigen Einflussfaktoren konnten wir aus der Analyse von zurückliegenden Bundestagswahlen ableiten und haben daraus ein statistisches Modell entwickelt, das den gemeinsamen Stimmenanteil der jeweiligen Regierungskoalition vorhersagt. So wird die Wahlentscheidung zwischen beliebig vielen Parteien zu einer Wahl für oder gegen die Regierung. Die Vorhersage des Stimmenanteils für die amtierende Regierungskoalition, die unser Modell normalerweise liefert, ist 2017 relativ sinnlos, weil Große Koalitionen nicht antreten, um wiedergewählt zu werden. Die Bildung einer Großen Koalition ist eine Notfalloption, die prinzipiell immer eine notwendige Sitzmehrheit im Bundestag sicher hat, wenn die Verhandlungen alternativer Koalitionsoptionen gescheitert sind. Daher sagen wir für die kommende Bundestagswahl den Stimmenanteil mehrerer denkbarer Regierungskoalitionen voraus. Mit Hilfe statistischer Analyseverfahren können wir die Gewichtung der Einflussfaktoren für die Stimmabgabe zu Gunsten der Regierungskoalition äußerst genau bestimmen und für eine Prognose der Bundestagswahl 2017 nutzen.

Sofern es sich bewahrheitet, dass die SPD weniger Sitze im neuen Bundestag erhält als die Union - und das ist derzeit sicher, wie Abbildung 1 zeigt - hat die Union alle Möglichkeiten, neben der Großen Koalition eine andere Regierung zu bilden. Die Wahrscheinlichkeit, eine Mehrheit für Schwarz-Gelb zu bekommen, liegt bei 92 Prozent, während eine Mehrheit für Schwarz-Grün mit 86 Prozent nur leicht unsicherer aussieht. Da es derzeit kein eindeutiges Koalitionssignal für eine dieser Konstellationen gibt (Gschwend et al. 2016; Gschwend et al. 2017), könnte es nach der Wahl zu spannenden Sondierungsgesprächen kommen. Die Union dürfte dann in der günstigen Lage sein, mehrere Mehrheitsoptionen zu besitzen. Das wäre eine exzellente Ausgangsposition für kommende Koalitionsverhandlungen. Solange keine dieser Zweiparteienkoalitionen eine rechnerische Mehrheit erlangt, ist die derzeit wahrscheinlichste Alternative zu einer Wiederauflage einer Großen Koalition, ähnlich wie nach der Bundestagswahl 2005, eine Jamaika-Koalition, die aber für die Bundesebene ein neues Koalitionsmodell darstellen würde und bisher nur auf der Landesebene ausprobiert wurde.

Wie sehen die Vorhersagen des Kanzlermodells für die SPD und die Aussichten auf eine Kanzlerschaft von Martin Schulz aus? Wenn die momentane Situation vom Juni noch im Herbst fortbesteht, ist die Antwort eindeutig. Es wird keinen Kanzler Schulz geben, weil er keine Machtoption haben wird. Für alle denkbaren Zweiparteienkoalitionen unter Führung der SPD und selbst die theoretisch denkbaren Dreiparteienkoalitionen, etwa eine Rot-Rot-Grüne Koalition („2RG-Koalition“) oder die sogenannte Ampel-Koalition aus SPD, FDP und den Grünen, wird keine Sitzmehrheit bekommen. Mit dem Kanzlermodell kann man jedoch nicht den Ausgang von Koalitionsverhandlungen vorhersagen. Hierfür gibt es spezielle Modelle (Bräuninger u. Debus 2008, 2012; Debus 2011). Es ist aber zu vermuten, dass FDP und die Grünen sich eher für eine Koalition mit der stärksten Fraktion als mit der zweitstärksten Fraktion aussprechen, wenn sie die Wahl haben. Selbst wenn es rechnerisch mögliche Koalitionsoptionen gäbe, die eine Kanzlerschaft von Schulz ermöglichen würden, ist dies solange unwahrscheinlich, wie die SPD nicht an der Union vorbeiziehen kann. Und das scheint derzeit unmöglich. 


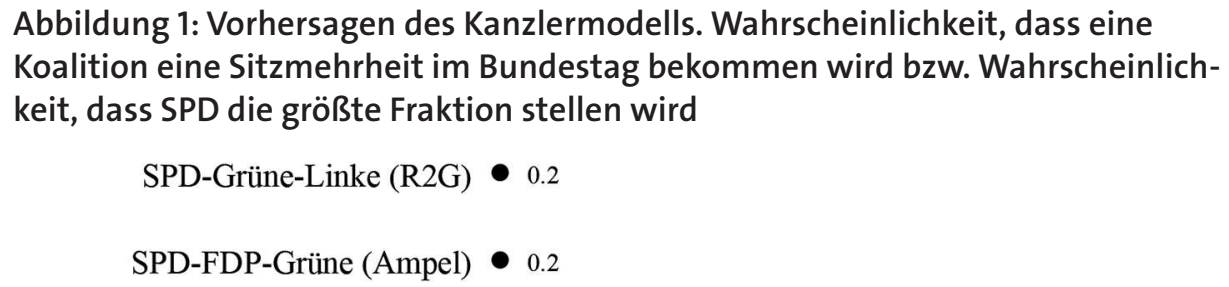

CDU/CSU-FDP-Grüne (Jamaika) $\longrightarrow 100$

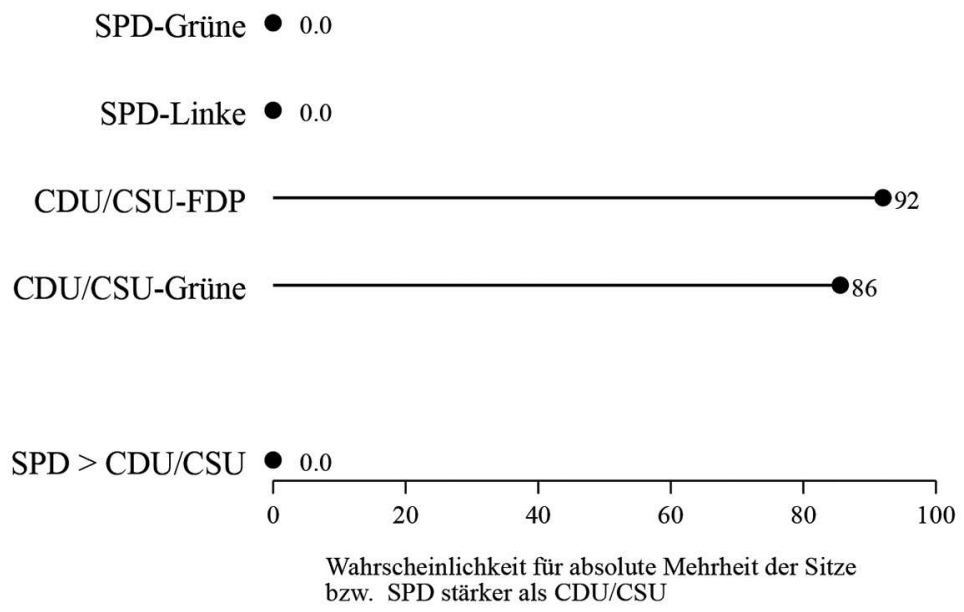

Quelle: Eigene Darstellung

Der wesentliche Grund, warum unser Modell die Aussicht auf einen Kanzler Schulz so niedrig schätzt, liegt darin, dass die deutschen Wählerinnen und Wähler, falls sie sich direkt für einen Kanzlerkandidaten entscheiden müssten, Merkel den Vorzug geben. Im Juni-Politbarometer der Forschungsgruppe Wahlen führt Merkel im Duell mit Schulz mit 59:31 Prozent, während die restlichen 10 Prozent noch unentschlossen sind. Bemerkenswert ist auch, dass der Rückhalt für Schulz in den eigenen Reihen bei 73 Prozent liegt, was so gar nicht zum „Mr. 100 Prozent" passt. Merkel hingegen scheint gegenüber Schulz bei den CDU/CSU-Anhängern sehr beliebt zu sein (95 Prozent). Andere Umfragen haben ähnliche Werte ergeben. Natürlich ist die Bundesrepublik kein Präsidialsystem, in dem der Präsident direkt gewählt wird. Das Kanzlermodell im Gleichklang mit vielen traditionellen Studien der Wahlforschung hat aber gezeigt, dass die individuelle Stimmabgabe bei Bundestagswahlen von den jeweiligen Präferenzen für einen Wunschkanzler beeinflusst wird.

Das einzige Argument, das gegen eine erneute Amtszeit von Merkel sprechen könnte, ist, dass nach drei Amtszeiten üblicherweise der Wunsch nach Veränderung in der Wählerschaft schon deutlich wird. Das ist nicht ungewöhnlich. Bei der jüngsten US-Präsidentschaftswahl profitierte der Republikaner Donald Trump von 
diesem Wunsch nach Veränderung nach zwei Amtszeiten des Demokraten Obama im Weißen Haus. Die Wechselstimmung und Trumps besseres Abschneiden in den Vorwahlen gegenüber Hillary Clinton machten eine solche Vorhersage bereits im Frühjahr 2016 möglich (Fox and Friends 2016). In Deutschland würde eine wachsende Unzufriedenheit mit der Kanzlerin, insbesondere gegenüber einem beliebter werdenden Herausforderer, auf eine mögliche Wechselstimmung in diesem Jahr schließen lassen. Die Situation Ende Februar dieses Jahres sah auch danach aus. Das Kanzlermodell bewertete zu jenem Zeitpunkt eine Kanzlerschaft von Martin Schulz als realistisch, sofern die Popularitätswerte über das Wahljahr stabil blieben (Norpoth u. Gschwend 2017). Dem ist mittlerweile aber nicht mehr so.

Im Folgenden werden wir nochmal die Grundzüge des Kanzlermodelles vorstellen und verdeutlichen, welche Informationen für die Vorhersagen benutzt werden. Danach beleuchten wir, wie gut das Kanzlermodell bisher als Vorhersagemodell funktioniert hat. Schließlich stellen wir im Detail die Vorhersagen für die kommende Bundestagswahl vor.

\section{Das Kanzlermodell}

Das Feld der wissenschaftlichen Wahlvorhersagen zeichnet sich durch eine ständig wachsende Anzahl von Ansätzen und Verfahren aus (Stegmaier u. Norpoth 2017). Ziel des Kanzlermodells ist es, den kombinierten Stimmanteil unterschiedlicher Koalitionen auf Basis von nur drei Faktoren vorherzusagen. Je nach Koalition haben diese Faktoren unterschiedliche Werte für die Vorhersage der kommenden Bundestagswahlergebnisse. Jede Vorhersage des kombinierten Stimmenanteils für Parteien einer Koalition ist das gewichtete Mittel dieser Faktoren. Die Gewichte dieser Faktoren sind für alle Vorhersagen im Rahmen der Bundestagswahl 2017 gleich. Sie wurden zuvor als Regressionskoeffizienten eines linearen Modells auf Basis von Daten der zurückliegenden Bundestagswahlen und Regierungskoalitionen geschätzt.

Die theoretische Motivation des Kanzlermodells haben wir an anderer Stelle (Gschwend u. Norpoth 2000, 2001, 2005a; Norpoth u. Gschwend 2010) bereits detaillierter beschrieben. Im Folgenden wollen wir die entscheidenden Punkte nochmals kurz zusammenfassen. Aufbauend auf Erklärungsfaktoren von Wahlentscheidungen, die schon die Klassiker der Wahlforschung wie The American Voter (Campbell et al. 1960, aktualisiert von Lewis-Beck et al. 2008) benutzten, setzt das Kanzlermodell besonders auf die Popularität der jeweiligen Spitzenkandidaten für das Amt des Bundeskanzlers. Da keine durchgehende Zeitreihe für den gesamten Zeitraum verfügbar ist, konstruieren wir nach dem Vorbild von Norpoth (1977) auf der Basis unterschiedlicher Frageformulierungen ein vergleichbares Messinstrument (Gschwend u. Norpoth 2000, S. 400). Für die kommende Bundestagswahl, wie auch bereits zuvor für die letzten Wahlen, messen wir diese Popularität mittels der Frage nach dem gewünschten Bundeskanzler aus dem Politbarometer der Forschungsgruppe Wahlen. ${ }^{2}$ Um Zufallsschwankungen

2 Weil das Kanzlermodell auch auf Ergebnisse von Umfragen angewiesen ist, kann man es auch als Ergänzung zu umfragebasierten Vorhersagemodellen sehen. 
zu glätten, bilden wir den Mittelwert der Politbarometer ein bis zwei Monate vor dem jeweiligen Wahltag (Norpoth u. Gschwend 2005, S. 375-377). Dieses Maß korreliert sehr stark $(0,7)$ mit dem gemeinsamen Stimmenanteil der Regierungsparteien bei den 17 Bundestagswahlen seit 1953.

Der zweite Faktor des Kanzlermodells beinhaltet die Idee, dass Parteien sich unter normalen Umständen auf ein gewisses Reservoir an Stimmen verlassen können. Viele Wähler fühlen sich typischerweise zu einer Partei besonders hingezogen. Man spricht daher von langfristig stabilen Parteibindungen bzw. von Parteiidentifikationen (Campbell et al. 1960). Anstelle von Individualdaten, die leider nicht in gleicher Weise über den gesamten Zeitraum verfügbar sind, benutzen wir als Maß für den langfristigen Wählerrückhalt der Regierungsparteien den durchschnittlichen Wahlerfolg bei den vorangegangenen drei Bundestagswahlen der Regierungsparteien. Wir greifen hierzu auf die offiziellen Wahlergebnisse der jeweiligen Parteien zurück. Auf diese Weise lässt sich der Effekt langfristiger Parteistärke von kurzfristig wirksamen Faktoren wie der Kanzlerunterstützung empirisch trennen (Norpoth u. Gschwend 2003b). Um ein vergleichbares Maß über die Zeit zu konstruieren, müssen wir jedoch die besondere Situation von 2005 und 2013 berücksichtigen. In diesen Wahlen sind relevante neue Parteien auf der politischen Bildfläche erschienen, die sich aber auf Kosten des langfristigen Wählerrückhalts der amtierenden Regierungskoalition etablierten und für die daher entsprechend korrigiert werden muss. ${ }^{3}$ Unser Maß für den langfristigen Wählerrückhalt der Regierungsparteien korreliert ebenfalls recht stark $(0,6)$ mit dem gemeinsamen Stimmenanteil der Regierungsparteien bei den 17 Bundestagswahlen seit 1953.

Schließlich haben wir als dritten und letzten Faktor des Kanzlermodells einen Effekt integriert, der die Dynamik von Wahlen abbildet. Eine theoretisch gut begründete These (etwa Campbell et al. 1960, S. 554; Paldam 1991, S. 19; Norpoth 2016) besagt, dass es Parteien Stimmen kostet, wenn sie Regierungsverantwortung tragen. Ein „zutiefst demokratisches Element“, wie der damalige Kanzler Schröder meinte, als wir ihm das Modell und seine Prognosen bei der Bundestagswahl 2002 vorstellten. Dieser mittelfristig wirksame Abnützungseffekt von Regierungsparteien führt dazu, dass es immer schwieriger werden sollte im Amt zu bleiben, je öfter der Amtsinhaber zur Wahl antritt. In diesem Sinn messen wir den Abnützungseffekt durch die Anzahl wie oft die kanzlerstellende Partei schon den Regierungschef gestellt hat. Bei den 17 Bundestagswahlen seit 1953 korreliert unser Maß wie erwartet negativ $(-0,38)$ mit dem gemeinsamen Stimmenanteil der Regierungsparteien.

3 Hofrichter u. Kunert (2009) zeigen, dass 76 Prozent der Stimmen für die Linke in 2005 von ehemaligen Wählern der vor der Wahl amtierenden Rot-Grünen Bundesregierung kamen. Der Stimmenzuwachs von 4,7 Prozentpunkten (Die Linke bekam 8,7 Prozent in 2005 und die PDS 4,0 Prozent in 2002) reduziert demnach den langfristigen Wählerrückhalt von Rot-Grün um 3,6 $(=4,7 * 0,76)$ Prozentpunkte, d.h., von eigentlich 46,1 Prozent auf 42,5 Prozent für 2005. Analog korrigieren wir für 2013 den langfristigen Wählerrückhalt von Schwarz-Gelb von 46,4 Prozent auf 43,9 Prozent um 2,5 Prozent $(=4,7 * 0,54)$. Die AfD trat erstmals an und gewann 4,7 Prozent der Zweitstimmen. Wählerwanderungsanalysen wiederum zeigen, dass 54 Prozent der AfD-Wähler aus dem Schwarz-Gelben Lager gekommen sind (siehe https://wahl.tagesschau.de/wahlen/201309-22-BT-DE/analyse-wanderung.shtml. [Zugegriffen: 05.02.2017]). 
Uns interessierte dabei besonders der gemeinsame Stimmenanteil der jeweiligen Regierungskoalition als eine für den Ausgang einer Wahl äußerst relevante Größe. Bekommt die Koalition eine Mehrheit, wird sie weiter regieren, ansonsten ist die amtierende Regierung abgewählt. Dies sagt unser Model üblicherweise vorher. Für die amtierende Regierung brauchen wir eine Notlösung. Große Koalitionen treten üblicherweise nicht zur Wiederwahl an. So auch nicht im September dieses Jahres. Wir erwarten daher, dass jede der amtierenden Regierungsparteien entsprechende Koalitionsoptionen nach der Wahl ausloten wird. Das war nach den vergleichbaren Wahlen von 1969 und 2009 nicht anders.

Auf diese Weise haben wir alle drei Faktoren des Kanzlermodells operationalisiert und als unabhängige Variablen eines linearen Modells spezifiziert. Anpassungen bei der Operationalisierung sind dabei notwendig, um die Vergleichbarkeit der Messstrategie über die Zeit herzustellen und damit die Annahme der kausalen Heterogenität am ehesten rechtfertigen zu können. Da wir ebenfalls den kombinierten Stimmenanteil der jeweiligen Regierungsparteien für alle 17 Bundestagswahlen von 1953 bis 2013 durch die offiziellen Wahlergebnisse erhalten, können wir auch diese Größe als abhängige Variable des Regressionsmodells spezifizieren. Die Schätzergebnisse zeigen wir in Tabelle 1.

Tabelle 1: Schätzwerte für die Parameter des Kanzlermodells

\begin{tabular}{|c|c|c|}
\hline \multirow[b]{2}{*}{ Unabhängige Variablen } & \multicolumn{2}{|c|}{$\begin{array}{c}\text { Abhängige Variable: } \\
\text { Stimmenanteil der Regierungsparteien } \\
(1953-2013)\end{array}$} \\
\hline & Parameter & $(\mathrm{SF})$ \\
\hline Kanzlerunterstützung & $0,39 * *$ & $(0,05)$ \\
\hline Langfristige Parteienstärke & $0,79 * * *$ & $(0,08)$ \\
\hline Abnützungseffekt & $-1,18 * *$ & $(0,32)$ \\
\hline Konstante & $-8,95 *$ & $(4,79)$ \\
\hline$\overline{\mathrm{R}}^{2}$ & \multicolumn{2}{|c|}{0,93} \\
\hline Wurzel der mittleren Fehlerquadratsumme & \multicolumn{2}{|c|}{1,49} \\
\hline $\mathrm{N}$ & \multicolumn{2}{|c|}{17} \\
\hline Lijung-Box Q (5 lags) & \multicolumn{2}{|c|}{2,08} \\
\hline
\end{tabular}

Anmerkungen: ${ }^{*} \mathrm{p}<0,1 ;{ }^{* *} \mathrm{p}<0,01 ;{ }^{* * *} \mathrm{p}<0,001$.

Quelle: eigene Darstellung.

Alle Parameter des Kanzlermodells erweisen sich als signifikant und haben das erwartete Vorzeichen. Die Größe der geschätzten Parameter für den Zeitraum 1953-2013 hat sich im Laufe der Jahre seit der Bundestagswahl 2002 kaum geändert. Das zeigt an sich schon, wie robust die Modellschätzungen sind, trotz der kleinen Fallzahl, auf der sie beruhen.

Die vom Modell vorhergesagten Stimmanteile weichen im Schnitt über die 17 Wahlen um nicht mehr als 1,5 Prozentpunkte vom tatsächlichen kombinierten Stimmenanteil der Regierungskoalitionen ab. Die erklärte Varianz des Modells liegt über 90 Prozent. Das ist vertrauenserweckend. Auf Basis dieser recht präzise geschätzten Koeffizienten können wir entsprechende Vorhersagen erhalten, indem 
wir konkrete Werte in die Regressionsgleichung einsetzen. Man kann allerdings bereits am relativ unpräzise geschätzten Koeffizienten der Konstante erkennen, dass die Modellprognosen trotz präzise geschätzter Faktoren noch einige Unsicherheit beinhalten werden.

\section{Wie gut war das Kanzlermodell bisher?}

Es ist beileibe nicht das erste Mal, dass wir eine Vorhersage für eine Bundestagswahl machen. Wir haben das Kanzlermodell zum ersten Mal für eine Prognose zum Ausgang der Bundestagswahl 2002 verwendet und haben es seither für jede Bundestagswahl angewandt. Zur Jahrtausendwende (Gschwend u. Norpoth 2000, 2001) haben wir mit einem solchen Modell absolutes Neuland betreten. Umso mehr freuen wir uns, dass es mittlerweile weitere Forschungsteams gibt, die über ihre Ansätze (Leininger u. Kayser 2017, Munzert et al. 2017) im aktuellen PVS-Forum berichten und so die Forschung zur Prognose und damit letztlich auch der Erklärung von Wahlen vorantreiben und vertiefen. Die akademische Wahlforschung, so meinen wir jedenfalls, hat sowohl Theorien und Modelle als auch darauf basierende Hypothesen anzubieten, die zur Prognose von Wahlergebnissen taugen (Norpoth u. Gschwend 2005). Fehlprognosen sind in diesem Zusammenhang lehrreich, weil sie zu einer Verbesserung der Theorie- als auch der Modellbildung führen können.

Die Modellvorhersagen des Kanzlermodells waren bisher recht genau und haben seit 2002 jedes Mal den Kanzler der nächsten Regierung korrekt vorhergesagt. Im Jahr 2002, zu einer Zeit, als Umfragen und Kommentatoren schon die amtierende Rot-Grüne Koalition unter Bundeskanzler Schröder abschrieben, sind wir mit unserer Prognose an die Öffentlichkeit getreten und haben eine Wiederwahl der amtierenden Regierungskoalition vorausgesagt. Die Vorhersage des Kanzlermodells, die drei Monate vor dem Wahltag veröffentlicht wurde, sagte den kombinierten Stimmenanteil Rot-Grün bis auf die erste Dezimalstelle (47,1 Prozent) korrekt voraus. Eine Leistung, die weder von einer Vorwahl-Umfrage noch von einem Exit-Poll am Wahlabend selbst (Gschwend u. Norpoth 2002, S. 16; Norpoth u. Gschwend 2003a) erreicht wurde. Bei der Wahl von 2005, der eine um ein Jahr verkürzte Legislaturperiode vorausging, hat unser Modell richtig vorausgesagt, dass die Rot-Grüne Koalition nicht wiedergewählt werden konnte, aber immer noch erfolgreich genug sein würde, um die Bildung einer Schwarz-Gelben Koalition (aus CDU/CSU und FDP) zu verhindern (Gschwend u. Norpoth 2005b, S. 30). Unsere Vorhersage für den kombinierten Stimmenanteil der amtierenden Regierungsparteien wich letztlich nur um 0,3 Prozentpunkte vom aktuellen Ergebnis ab. Das war genauer als alle Umfragen zu der Zeit und besser als fast alle 18-Uhr-Prognosen am Wahltag selbst (Gschwend u. Norpoth 2005a).

Zur Bundestagswahl 2009 haben wir für die amtierende Große Koalition unter Bundeskanzlerin Merkel vorausgesagt, dass eine neue Koalition, bestehend aus CDU/CSU und FDP, genügend Stimmen gewinnen würde, um sich die Mehrheit der Sitze im Bundestag zu sichern (Gschwend u. Norpoth 2009a). Dies geschah auch so. Für die letzte Bundestagswahl 2013 schließlich sagte unser Modell vor- 
aus, dass Merkels Regierungskoalition genügend Stimmen bekommen würde, um im Amt zu bleiben (Norpoth u. Gschwend 2013). Nur 0,2 Prozentpunkte trennten unsere Prognose vom tatsächlichen Ergebnis. Diese 0,2 Prozent waren genau der Anteil, mit dem die FDP, die 4,8 Prozent der Stimmen erreichte, die 5-ProzentHürde verpasste.

\section{Die Vorhersage für 2017}

Für die Vorhersage der kommenden Bundestagswahl benutzen wir dieselben Operationalisierungsstrategien zur Messung der Kanzlerunterstützung und der langfristigen Parteienstärke wie zuvor. Die entsprechenden Werte der langfristigen Parteienstärke sind in Tabelle 2 zusammengefasst.

Tabelle 2: Benutzte Werte der langfristigen Parteienstärke zur Vorhersage möglicher Koalitionen bei der Bundestagswahl 2017

\begin{tabular}{lc}
\hline Koalition & $\begin{array}{c}\text { langfristige } \\
\text { Parteienstärke (in \%) }\end{array}$ \\
\hline CDU/CSU-FDP & 46,6 \\
CDU/CSU-Grüne & 45,9 \\
CDU/CSU-FDP-Grüne & 55,6 \\
SPD-Linke-Grüne & 46,4 \\
SPD-FDP-Grüne & 46,4 \\
SPD-Grüne & 36,7 \\
SPD-Linke & 37,4 \\
SPD & 27,6 \\
CDU/CSU & 36,8 \\
\hline
\end{tabular}

Quelle: eigene Darstellung.

Für den Abnützungseffekt haben wir die absolute Anzahl der Amtsperioden der Regierung (statt der logarithmierten Anzahl wie bei der Vorhersage zur Bundestagswahl 2013) genommen, um so auch den Stimmenanteil für mögliche Regierungskoalitionen eines neuen Kanzlers (Martin Schulz) vorhersagen zu können. Für den Stimmenanteil von Koalitionen unter der Führung von Schulz sollte noch kein Abnützungseffekt wirksam sein, da die Koalition ja noch gar nicht im Amt ist. Der entsprechende Wert ist daher ,0'. Jede Merkel-geführte Regierungskoalition muss dagegen mit einem Abnützungseffekt von ,3', das heißt mit einem Malus aufgrund langjähriger Regierungsbeteiligung im Durchschnitt von mehr als 3,5 Prozentpunkten $(=3 * 1,18)$ rechnen.

Um eine vorläufige Prognose zu erstellen, nehmen wir den neuesten Wert für die Kanzlerunterstützung, den die Forschungsgruppe Wahlen im Politbarometer vom Juni (02.06.2017) veröffentlicht hat. Zu diesem Zeitpunkt wünschen sich 59 Prozent der Befragten des Politbarometers weiterhin Angela Merkel als Kanzlerin. Martin Schulz kommt dabei nur auf 31 Prozent. Lässt man die Zahl der Unent- 
schlossenen außer Acht, bedeutet das 66 Prozent für Merkel als Wunschkanzlerin und 34 Prozent für Schulz als Wunschkanzler. ${ }^{4}$

Es ist natürlich schon jetzt klar, dass die Große Koalition nach der Bundestagswahl weiter regieren könnte, wenn das die Partner wollen. Dazu braucht es sicherlich kein Modell. Es wird aber nur dazu kommen, wenn sich die Parteien auf keine andere Koalition einigen können. Eine andere Frage ist aber, wer den Kanzler stellen wird. Das scheint eine der spannenden Fragen dieser Wahl zu sein. Wird die SPD mit einem Kanzlerkandidat Martin Schulz stärker sein als die Union mit Bundeskanzlerin Angela Merkel? Das ist gerade auch deshalb wichtig, weil wir aus der Forschung zu Koalitionsbildung wissen, dass FDP und Grüne vermutlich eher mit der stärksten Fraktion eine Koalition bilden werden.

Abbildung 2 gibt einen Überblick über die unterschiedlichen Prognosen des Kanzlermodells. Für eine intuitivere Kommunikation der Unsicherheit, die unseren Vorhersagen innewohnt, benutzen wir zwei Arten von Unsicherheitsbereichen. Die Länge der traditionellen 95 Prozent-Konfidenzintervalle, die als dünne Linien abgetragen sind, verdeutlichen, mit wieviel Unsicherheit diese Prognosen behaftet sind. Da diese Werte auf Simulationen beruhen, können wir sie intuitiv interpretieren. Diese Konfidenzintervalle geben an, dass mit einer Wahrscheinlichkeit von 95 Prozent der Ausgang der Wahl in diesem Bereich sein wird. Ein intuitiveres Verständnis wie groß diese Unsicherheiten tatsächlich sind bieten 84 Prozent Konfidenzintervalle an, die durch die dickeren Linien gekennzeichnet sind. Diese geben an, dass die Wahrscheinlichkeit, dass das tatsächliche Ergebnis am Wahltag in diesem Bereich sein wird in etwa so groß ist wie beim einmaligen Würfeln keine , $6^{\prime}$ zu erzielen (genauer: $1-1 / 6 \approx 0,84$ ). Wir wissen alle aus eigener Erfahrung, dass eine Zahl zwischen ,1' und ,5' zu würfeln recht wahrscheinlich, trotzdem aber nicht völlig sicher ist.

Momentan sagt das Kanzlermodell voraus, dass eine Jamaika-Koalition (CDU/ CSU-FDP-Grüne) bei der kommenden Bundestagswahl einen Stimmenanteil von 56,9 Prozent erhalten wird. Daher ist auch klar, dass diese Koalition (neben der Großen Koalition) auch die besten Aussichten hat, eine Mehrheit der Sitze zu erhalten. Trotz Vorhersageunsicherheit sollte dieser Zweitstimmenanteil genügen, um eine Sitzmehrheit im neuen Bundestag zu erreichen.

Welcher Stimmenanteil ist ausreichend, um die Mehrheit der Sitze im Bundestag zu gewinnen und so eine Regierung zu bilden? Unter der Annahme, dass die Stimmen für alle anderen Parteien, die keine Sitze im Bundestag erhalten, mindestens fünf Prozent betragen, kann man davon ausgehen, dass die Hälfte von 95 (= 100

4 Im Nachgang zur Bundestagswahl 2009 identifizierten wir für den jeweiligen Kanzler einen „Großer Koalitions-Bonus" (Gschwend u. Norpoth 2009b) bei Wahlen mit einer Großen Koalition als Amtsinhaberin, der sonst nicht zu erwarten ist. Dieser Bonus muss folglich bei der Operationalisierung der Werte für 1969 und 2009 herausgerechnet werden, um ein vergleichbares Maß über die Zeit zu gewährleisten. 2017 wäre ebenfalls eine solche Korrektur fällig. Allerdings ist ein solcher Bonus derzeit (Stand Juni 2017) nicht festzustellen. Die Popularität von Schulz unter den SPD Anhängern ist noch nicht so niedrig, wie man üblicherweise erwarten könnte, wäre ein „Großer Koalitions-Bonus“ für Merkel wirksam. Das liegt vermutlich daran, dass mit Martin Schulz ein bundespolitischer „Outsider“ zum Herausforderer von Angela Merkel nominiert wurde und sich so der „Großer Koalitions-Bonus“ in der Popularität der amtierenden Bundeskanzlerin bisher nicht einstellt. 
- 5) Prozent der Stimmen, also 47,5 Prozent ausreichen sollten, um eine Mehrheit der Sitze im neuen Bundestag zu sichern. Die beiden Dreiparteienkoalitionen unter der Führung der SPD, Ampel und R2G sehen wir derzeit bei 40,8 Prozent und damit systematisch niedriger als 47,5 Prozent. Beide Koalitionen sieht das Kanzlermodell derzeit als nicht mehrheitsfähig. Schwarz-Grün hingegen liegt bei 49,3 Prozent und Schwarz-Gelb sogar bei 49,8 Prozent. Beide Koalitionsoptionen sind zwar nicht systematisch größer als 47,5 Prozent nach herkömmlichen Standard, da das 95 Prozent Konfidenzintervall den Wert ,47,5' beinhaltet. Die Wahrscheinlichkeit, dass diese Koalitionen eine rechnerische Mehrheit erhalten, ist trotzdem sehr hoch, wie Abbildung 1 bereits verdeutlicht hat. Die Wahrscheinlichkeit, dass Schwarz-Gelb eine Sitzmehrheit im Herbst erhält ist derzeit tatsächlich in etwa so groß wie die Wahrscheinlichkeit, keine ,6' zu würfeln - also schon recht wahrscheinlich, aber nicht ganz sicher. Für alle anderen aufgeführten Koalitionskonstellationen in Abbildung 2 sehen wir keine Mehrheit der Sitze im neuen Bundestag, da die vorhergesagten Werte systematisch kleiner als 47,5 Prozent sind.

\section{Abbildung 2: Vorhersagen für die kommende Bundestagswahl basierend auf dem Kanzlermodell}

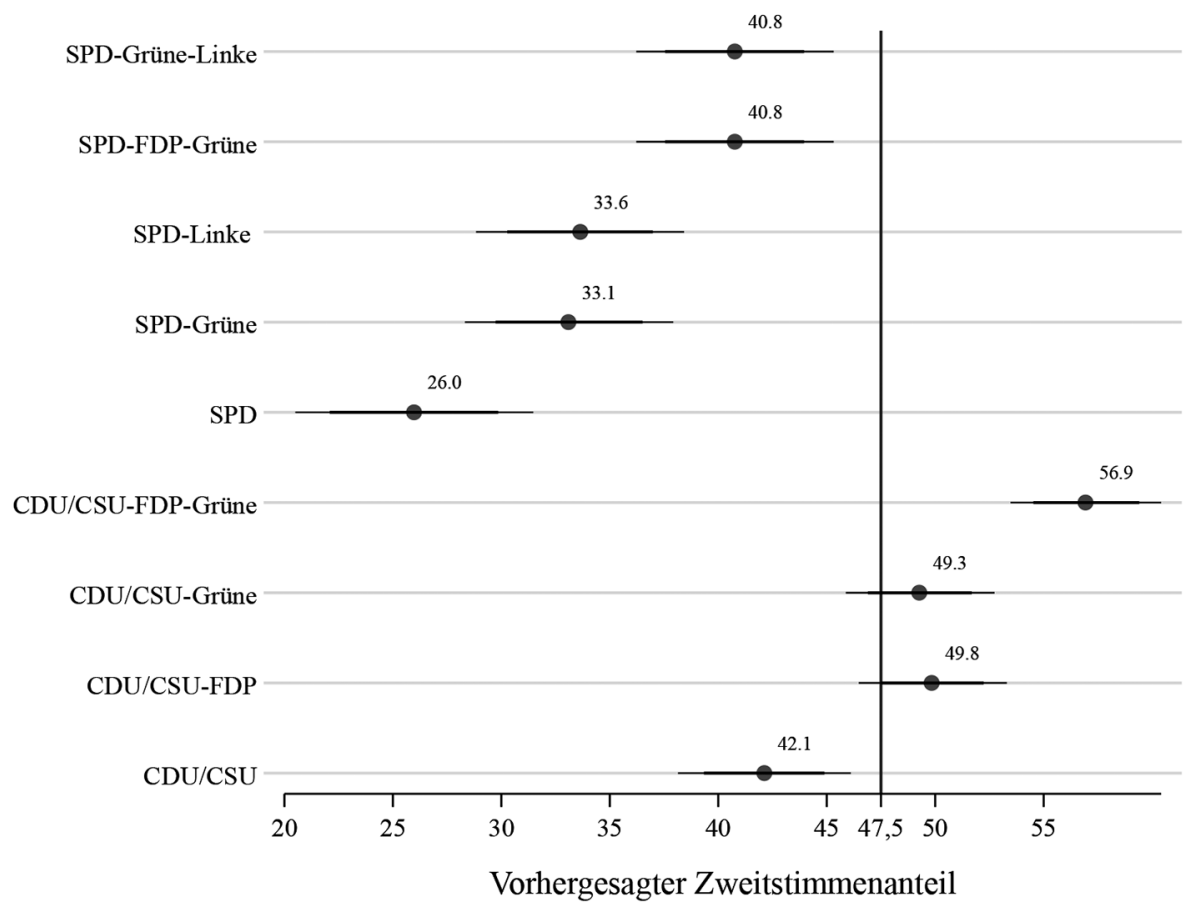

Quelle: eigene Darstellung.

Woher kommt diese Unsicherheit und wie wird sie in unserer Analyse berücksichtigt? Jeder auf Basis des Modells vorhergesagte Wert beinhaltet beide Arten von 
Unsicherheit. Zum einen ist jeder Regressionsparameter mit Schätzunsicherheit behaftet, die letztlich bereits durch die Angabe von Standardfehlern, wie etwa in Tabelle 1, dokumentiert wird. Zum anderen kommt für die konkrete Vorhersage von Wahlen hinzu, dass wir uns nie sicher sein können - selbst dann nicht, wenn wir alle Regressionsparameter genau kennen würden. Diese grundsätzliche Unsicherheit kommt also hinzu, weil jede konkrete Wahlvorhersage zusätzlich von dem Einfluss unzähliger Zufallsereignisse abhängt, die vom Verfassen dieser Zeilen bis zum Wahltag noch auftreten werden. Denkbar wären etwa politische oder wirtschaftliche Krisensituationen (Börsensturz, Terroranschlag). Diese zufälligen Ereignisse beeinflussen die Sicherheit unserer Vorhersagen, sind aber nicht systematisch Teil des Kanzlermodells. Um beide Arten von Unsicherheiten für die Vorhersage der kombinierten Zweitstimmenanteile für Koalitionen zu berücksichtigen, aber auch um vorherzusagen, wie wahrscheinlich es ist, dass eine bestimmte Koalition eine Mehrheit der Sitze im neuen Bundestag erhalten wird, verwenden wir einen parametrischen Bootstrap-Ansatz (King et al. 2000). Dabei simulieren wir 10.000 Vorhersagen für die kommende Bundestagswahl. Der Trick dabei ist, dass die Variabilität unserer simulierten Wahlvorhersagen beide Arten von Unsicherheit widerspiegelt. Je sicherer die Regressionskoeffizienten für unsere Vorhersage geschätzt wurden, desto geringer ist die Variabilität der Vorhersagen für die kommende Bundestagswahl. ${ }^{5}$

Wenn wir also sagen, wie eingangs in Abbildung 1, dass eine Schwarz-Gelbe Koalition eine 92-prozentige Wahrscheinlichkeit hat, eine Mehrheit zu gewinnen, dann heißt das, dass von 10.000 simulierten Vorhersagen für die kommende Bundestagswahl 9.200 Vorhersagen einen kombinierten Stimmenanteil liefern, der größer als 47,5 (= 95/2) Prozent ist. Die Angabe einer Wahrscheinlichkeit eine Mehrheit der Sitze zu erlangen, ist dabei eine Möglichkeit, wie Unsicherheit in Prognosen kommuniziert werden kann. Abbildung 2 zeigt, dass wir sowohl für eine Ampel-Koalition wie auch für eine R2G-Koalition einen geringeren Stimmenanteil von 40,8 Prozent erwarten. Die Aussicht auf eine Mehrheit von Sitzen steht für beide Koalitionen bei nur 0,2 Prozent. Das ist daher praktisch ausgeschlossen.

Wenn die kommende Bundestagswahl so ausgeht wie vorhergesagt, dann hat Kanzlerin Merkel derzeit neben der Großen Koalition viele Alternativen, um an der Regierung zu bleiben. Am wahrscheinlichsten ist eine Schwarz-Gelbe Koaliti-

5 Technisch gesehen berücksichtigen wir die Schätzunsicherheit der Regressionsparameter dadurch, dass wir 10.000 Zufallsziehungen von einer multivariaten Normalverteilung verwenden, deren Mittelwert der Vektor der geschätzten Regressionskoeffizienten bzw. deren Varianz der geschätzten Varianz-Kovarianz-Matrix des Kanzlermodells entspricht. Auf Basis konkreter Werte für die drei unabhängigen Variablen des Kanzlermodells für 2017 lassen sich nun entsprechend 10.000 verschiedene Zweitstimmenanteile berechnen. Diese Vorhersagen variieren umso weniger, je genauer die Regressionskoeffizienten geschätzt wurden. Um die grundsätzliche Unsicherheit ebenfalls in die Vorhersagen zu integrieren, wird zu jedem der Zweitstimmenanteile noch ein Fehlerterm addiert, der zufällig aus einer Normalverteilung gezogen wird, deren Varianz der geschätzten Varianz des Kanzlermodells entspricht. So erhält man nun eine Verteilung von verschiedenen Wahlvorhersagen für jedes gewählte Szenario, was durch die Werte der unabhängigen Variablen für 2017 definiert ist und variiert, weil es sowohl die Schätzunsicherheit als auch die grundsätzliche Unsicherheit beinhaltet. Der Mittelwert dieser Verteilung ist die Vorhersage des Kanzlermodells. Die Variabilität der Verteilung dieser Wahlvorhersagen spiegelt also beiden Arten von Unsicherheiten wider. 
on, aber auch eine rechnerische Mehrheit für eine Schwarz-Grüne Koalition ist denkbar. Sofern die SPD nicht noch die CDU/CSU überflügeln kann, braucht der sozialdemokratische Kanzlerkandidat Martin Schulz in jedem Fall die Grünen und einen weiteren Koalitionspartner, um selbst Kanzler zu werden. Falls es aber weder für Schwarz-Gelb noch für Schwarz-Grün für eine Sitzmehrheit reichen sollte, würde es eher zu einer Jamaika-Koalition kommen als zu einer Ampel-Koalition. Die stärkere der beiden Volksparteien wird mehr Optionen im anstehenden Koalitionspoker haben. Eine entscheidende Frage ist also: Welche Partei stellt die stärkste Fraktion im neuen Bundestag? Unser Modell kann in Abhängigkeit von der Popularität der Kanzlerin gegenüber ihrem Herausforderer diese Frage beantworten.

Abbildung 3: Wer wird die stärkste Fraktion im neuen Bundestag? Vorhergesagter Zweitstimmenanteil des Kanzlermodells für CDU/CSU bzw. SPD sowie Wahrscheinlichkeit, dass die SPD stärkste Fraktion wird

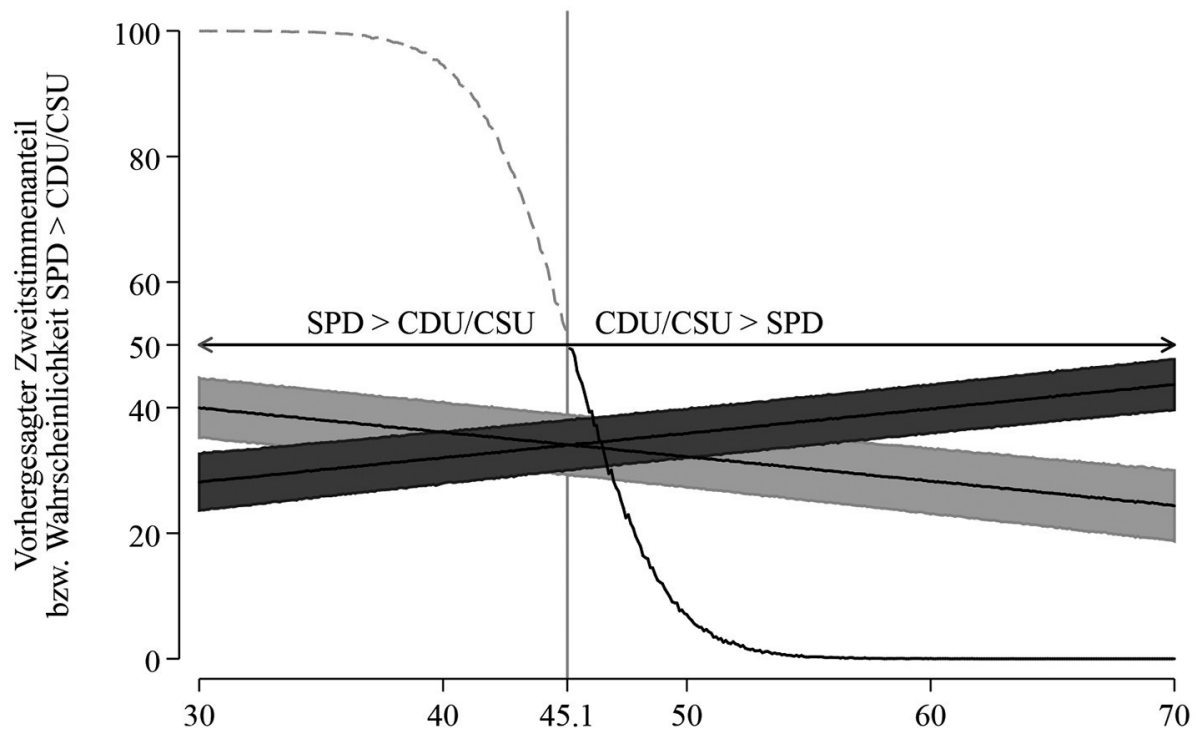

Kanzlerunterstützung Merkel [in \%]

Quelle: eigene Darstellung.

Abbildung 3 zeigt den vom Kanzlermodell vorhergesagten Zweitstimmenanteil für CDU/CSU und SPD in Abhängigkeit der Kanzlerunterstützung einen Monat vor der Wahl. Diese ist zum Zeitpunkt der Abgabe des Manuskripts noch nicht bekannt. Wir sehen, dass, je populärer Merkel gegenüber Schulz ist, das Kanzlermodell für die CDU/CSU mehr Zweitstimmen vorhersagt (die aufsteigende Linie mit dunkelgrauen $95 \%$-Konfidenzintervallen) und umgekehrt weniger Zweitstimmen für die SPD (die absteigende Linie mit hellgrauen $95 \%$-Konfidenzinter- 
vallen). Solange die Kanzlerin aber eine Unterstützung von mindestens mehr als 45,1 Prozent erhält, sieht unser Modell die Union stärker als die SPD. Gegenwärtig steht Merkel sogar bei 66 Prozent. Insofern ist es praktisch unmöglich für die SPD stärkste Fraktion zu werden, solange Merkel im Vergleich zu Schulz derart populär ist. Die entsprechende Kurve der Wahrscheinlichkeit, dass der Zweitstimmenanteil der SPD größer ist als der der CDU/CSU, ist bei 0 Prozent.

Die Wahrscheinlichkeit, dass die SPD statt der Union stärkste Fraktion wird, ist bis zu einer Unterstützung Merkels von 45 Prozent eher wahrscheinlich. Martin Schulz, als Herausforderer, muss also mindestens 55 Prozent an Unterstützung in der Kanzlerfrage bekommen, damit das Kanzlermodell die Wahrscheinlichkeit über 50 Prozent sieht (was die gestrichelte Linie markiert), dass die SPD stärkste Fraktion im neuen Bundestag wird. Falls sich ein Kopf-an-Kopf-Rennen in der Kanzlerfrage entwickelt, werden die entsprechenden vorhergesagten Wahrscheinlichkeiten, ob die SPD oder die Union mehr Stimmen erhalten wird, zum Teil erheblich schwanken, wie an der Steilheit der Kurve in dem Bereich zwischen 40 Prozent und 50 Prozent in der obigen Abbildung zu sehen ist. Bleibt die derzeitige Popularität von 66 Prozent bis zum August erhalten, steht einer Wiederwahl der amtierenden Kanzlerin mit der Union als stärkster Fraktion im neuen Bundestag, unabhängig von jedweden Koalitionssignalen, die etwa bei früheren Wahlen eine Rolle gespielt haben (Gschwend et al. 2016, Gschwend et al. 2017), nichts entgegen. Jede CDU-geführte Regierung würde allerdings bei der ersten Bundestagswahl nach der kommenden Wahl im September gegen eine erhebliche Wechselstimmung ankämpfen müssen, da der entsprechende Abnützungseffekt nun auf ,4' steigen würde. Unser Kanzlermodell macht eine solche Vorhersage ebenfalls heute schon möglich.

\section{Literatur}

Bräuninger, Thomas, und Marc Debus. 2008. Der Einfluss von Koalitionsaussagen, programmatischen Standpunkten und der Bundespolitik auf die Regierungsbildung in den deutschen Ländern. Politische Vierteljahresschrift 49(2): 309-338. DOI: 10.1007/ s11615-008-0101-6.

Bräuninger, Thomas, und Marc Debus. 2012. Parteienwettbewerb in den deutschen Bundesländern. Wiesbaden: VS Verlag für Sozialwissenschaften.

Campbell, Angus, Philip E. Converse, Warren E. Miller, und Donald E. Stokes. 1960. The American Voter. New York: Wiley. DOI: 10.1126/science.132.3420.138.

Debus, Marc. 2011. Parteienwettbewerb, Regierungsbildung und Ergebnisse der Koalitionsverhandlungen nach der Bundestagswahl 2009. In Die Parteien nach der Bundestagswahl 2009, Hrsg. Oskar Niedermayer, 281-306. Wiesbaden: VS Verlag für Sozialwissenschaften.

Fox and Friends. 2016. Political Science Professor: 97-99\% Chance That Trump Will Be President. http://insider.foxnews.com/2016/02/26/political-science-professor-97-99chance-trump-will-be-president. Zugegriffen: 05.05.2017. 
Gschwend, Thomas, und Helmut Norpoth. 2000. Soll und Haben: Die deutsche Wählerschaft rechnet mit den Parteien ab. In 50 Jahre Empirische Wablforschung in Deutschland. Entwicklung, Befunde, Perspektiven, Daten, Hrsg. Markus Klein, Wolfgang Jagodzinski, Ekkehard Mochmann, und Dieter Ohr, 389-409. Wiesbaden: Westdeutscher Verlag. DOI: 10.1007/978-3-322-90180-4_16.

Gschwend, Thomas, und Helmut Norpoth. 2001. 'Wenn am nächsten Sonntag ...': Ein Prognosemodell für Bundestagswahlen. In Wablen und Wähler: Analysen aus Anlass der Bundestagswabl 1998, Hrsg. Hans-Dieter Klingemann, und Max Kaase, 473-499. Wiesbaden: Westdeutscher Verlag.

Gschwend, Thomas, und Helmut Norpoth. 2002. Die beste Wahlprognose. Warum eine Methode erfolgreicher war als alle anderen. Financial Times Deutschland, 24. September 2002.

Gschwend, Thomas, und Helmut Norpoth. 2005a. Prognosemodell auf dem Prüfstand: Die Bundestagswahl 2005. Politische Vierteljahresschrift 46(4): 682-688. DOI: 10.1007/ s11615-005-0308-8. DOI: 10.1007/s11615.005.0308-8.

Gschwend, Thomas, und Helmut Norpoth. 2005b. Schröder lebt. Financial Times Deutschland, 3. Juni 2005.

Gschwend Thomas, und Helmut Norpoth. 2009a. Schwarz-Gelb wird gewinnen. http://blog. zeit.de/zweitstimme/2009/08/24/schwarz-gelb-wird-gewinnen/. Zugegriffen: 20.04.2017.

Gschwend Thomas, und Helmut Norpoth. 2009b. Das Kanzlermodell bei der Wahl 2009: Diesmal kein Volltreffer. http://blog.zeit.de/zweitstimme/2009/10/14/das-kanzlermodell-bei-der-wahl-2009-diesmal-kein-volltreffer/. Zugegriffen: 21.04.2017.

Gschwend, Thomas, Lukas Stoetzer, und Steffen Zittlau. 2016. What Drives Rental Votes? How Coalitions Signals Facilitate Strategic Coalition Voting. Electoral Studies 44: 293-306. DOI: 10.1016/j.electstud.2016.06.011.

Gschwend, Thomas, Michael F. Meffert, und Lukas F. Stoetzer. 2017. Weighting Parties and Coalitions: How Coalition Signals Influence Voting Behavior. Journal of Politics 79(2): 642-55. DOI: 10.1086/688678.

Hofrichter, Jürgen, und Michael Kunert. 2009. Wählerwanderung bei der Bundestagswahl 2005: Umfang, Struktur und Motive des Wechsels. In Wablen und Wähler: Analysen aus Anlass der Bundestagswabl 2005, Hrsg. Oscar W. Gabriel, Bernhard Weßels, und Jürgen W. Falter, 228-250. Wiesbaden: VS Verlag für Sozialwissenschaften. DOI: 10.1007/978-3-531-91666-8_9.

King, Gary, Michael Tomz, und Jason Wittenberg. 2000. Making the Most of Statistical Analyses: Improving Interpretation and Presentation. American Journal of Political Science 44(2): 347-61. DOI: 10.2307/2669316.

Leininger, Arndt, und Mark A. Kayser. 2017. Eine länderbasierte Prognose zur Bundestagswahl 2017. Politische Vierteljahresschrift 58(3): 407-417.

Lewis-Beck, Michael S., William G. Jacoby, Helmut Norpoth, und Herbert F. Weisberg. 2008. The American Voter Revisited. Ann Arbor, MI: University of Michigan Press.

Munzert, Simon, Lukas Stötzer, Thomas Gschwend, Marcel Neunhoeffer, und Sebastian Sternberg. 2017. Zweitstimme.org. Ein strukturell-dynamisches Vorhersagemodell für Bundestagswahlen. Politische Vierteljahresschrift 58(3): 418-441.

Norpoth, Helmut. 1977. Kanzlerkandidaten. Wie sie vom Wähler bewertet werden und seine Wahlentscheidung beeinflussen. Politische Vierteljahresschrift 18(2/3): 551-572. 
Norpoth, Helmut. 2016. Primary Model Predicts Trump Victory. Political Science and Politics 49(4): 655-658. DOI: 10.1017/S1049096516001323.

Norpoth, Helmut, und Thomas Gschwend. 2003a. Against All Odds? The Red-Green Victory. German Politics and Society 21(1): 15-34. DOI: 10.3167/104503003782353619.

Norpoth, Helmut, und Thomas Gschwend. 2003b. Politbarometer und Wahlprognosen: Die Kanzlerfrage. In Politbarometer, Hrsg. Andreas Wüst, 109-123. Opladen: Leske + Budrich.

Norpoth, Helmut, und Thomas Gschwend. 2005. Mit Rot-Grün ins Schwarze getroffen: Prognosemodell besteht Feuertaufe. In Wablen und Wähler. Analysen aus Anlass der Bundestagswahl 2002, Hrsg. Jürgen W. Falter, Oscar W. Gabriel, und Bernhard Weßels, 371-387. Wiesbaden: VS Verlag für Sozialwissenschaften.

Norpoth, Helmut, und Thomas Gschwend. 2010. The chancellor model: Forecasting German elections. International Journal of Forecasting 26(1): 42-53. DOI: org/10.1016/j. ijforecast.2009.02.008.

Norpoth, Helmut, und Thomas Gschwend. 2013. Chancellor Model Picks Merkel in 2013 German Election. Political Science and Politics 46(3): 481-482. DOI: org/10.1017/ S1049096513000802.

Norpoth, Helmut, und Thomas Gschwend. 2017. Chancellor Model Predicts a Change of the Guard. Political Science and Politics 50(3): 686-688. DOI: 10.1017/S1049096517000415.

Paldam, Martin. 1991. How Robust is the Vote Function? A Study of Seventeen Nations over Four Decades. In Economics and Politics. The Calculus of Support, Hrsg. Helmut Norpoth, Michael S. Lewis-Beck, und Jean Dominique Lafay, 9-31. Ann Arbor, MI: The University of Michigan Press.

Stegmaier, Mary, und Helmut Norpoth. 2017. Election Forecasting. In Oxford Bibliographies in Political Science, Hrsg. Rick Valelly, New York, NY: Oxford University Press. DOI: 10./1093/OBO/9780199756223-0023.

\section{Autorenangaben}

Prof. Thomas Gschwend, Ph.D.

Professor für Politikwissenschaft, quantitative sozialwissenschaftliche Methoden

Universität Mannheim

68131 Mannheim

E-Mail: gschwend@uni-mannheim.de

Prof. Helmut Norpoth, Ph.D.

Professor für Politikwissenschaft

Stony Brook University

Stony Brook, New York 11794-4392

E-Mail: helmut.norpoth@stonybrook.edu 


\title{
Eine länderbasierte Prognose zur Bundestagswahl 2017
}

\author{
Arndt Leininger und Mark A. Kayser
}

\begin{abstract}
A Länder-based Forecast of the 2017 German Bundestag Election
Abstract: When elections are distant, polls are poor predictors. Too few voters are paying attention and too much can change before election day. Structural models can establish baseline expectations but suffer from high uncertainty and underspecification imposed by small samples. We present an early forecast of the 2017 Bundestag election results for individual parties that leverages economic and political data as well as state parliament (Landtag) election results in the German states (Länder) to sidestep these shortcomings. A linear random effects model provides our estimates. Länder elections are dispersed over the calendar and offer the advantage of capturing both actual voter preferences and new political issues. We argue that this approach offers a promising method for early forecasts when polls are not informative.
\end{abstract}

Keywords: Elections, forecasting, parties, Germany

Stichworte: Wahlen, Prognosen, Parteien, Deutschland

\section{Einleitung 1}

Im Gegensatz zu den USA und einigen anderen föderalen Staaten sind die Wahlen auf Landesebene in Deutschland über den Kalender verstreut. Jede dieser Wahlen zieht bundesweit Aufmerksamkeit auf sich und kann als Gradmesser der Wählerunterstützung angesehen werden. Dies gilt nicht nur für die jeweilige Landesregierung, sondern ebenso für die Bundesregierung in Berlin. Wir nutzen diese subnationalen Wahlen, um auf Basis eines strukturellen Modells eine Prognose für die Bundestagswahl 2017 zu erstellen. Damit testen wir nicht nur die tatsächliche Vorhersagekraft von Landtags- für Bundestagswahlen, sondern liefern zudem einen Ansatz mit dem sich zwei häufige Probleme struktureller Modelle umgehen lassen: einerseits die geringe Größe der Stichproben aufgrund der begrenzten Anzahl vorangegangener Wahlen und andererseits deren Unvermögen, Veränderungen zu erfassen, die im Zeitraum nach der vorigen nationalen Wahl aufgetreten sind.

Durch die Verwendung der Ergebnisse von Landtagswahlen werden diese beiden Mängel zumindest teilweise behoben. Denn zum einen erhöht sich die Anzahl der Beobachtungen um das 16-fache und zum anderen werden Informationen zugänglich, die zeitlich näher an der betreffenden Bundestagswahl liegen. Es mag zwar sein, dass Wahlumfragen und deren Aggregation den Ausgang der Wahlen kurz vor ihrem Stattfinden präziser vorhersagen können, mit zunehmendem zeit-

1 Wir bedanken uns ganz herzlich bei Julian Frinken, Markus Hembes, Thorsten Faas, Robert Welz sowie den Teilnehmerinnen und Teilnehmern der Tagung der Sektion Methoden der DVPW für sehr hilfreiche Kommentare und Hinweise. Alle möglicherweise verbleibenden Fehler sind natürlich allein den Autoren zuzuschreiben. 
lichen Abstand zur Wahl und steigendem Desinteresse der Wähler gegenüber den politischen Parteien und deren Inhalten verringert sich diese Überlegenheit jedoch, bis sie sich schließlich in ihr Gegenteil verkehrt (Erikson u. Wlezien 2012, 2014). Strukturelle Modelle sind nicht nur bessere Prädikatoren, wenn die Wahl, deren Ausgang prognostiziert werden soll, zeitlich noch weit entfernt ist. Sie haben zudem den Vorteil, dass sie tatsächliche Prognosen erlauben. Obwohl Wahlumfragen und deren Aggregation in der Öffentlichkeit häufig als Prognosen wahrgenommen werden (Blumenthal 2014), handelt es sich bei ihnen eher um prinzipiell vergängliche Momentaufnahmen.

Unsere Prognose, die wir Mitte Mai 2017 für die Wahl am 24. September abgaben, wird womöglich weiter von dem tatsächlichen Wahlergebnis abweichen als Umfragen, die kurz vor dem Wahltag durchgeführt werden. Dieser Umstand sollte jedoch keinen Zweifel daran aufkommen lassen, dass Strukturmodelle auch dann noch von Wert sind, wenn eine Wahl kurz bevorsteht. Denn da strukturelle Modelle im Gegensatz zu Umfragen theoriebasiert sind und auf historischen Daten beruhen, erfüllen sie die wichtige Funktion, Erwartungen aufzustellen, mit denen die tatsächlichen Resultate schließlich verglichen werden können. Durch die Schätzung eines Prognosemodells auf Grundlage des Zusammenhangs zwischen vorangegangenen Wahlen und Kovariaten sagen Strukturmodelle im Kern voraus, wie ein durchschnittlicher Kandidat mit einer durchschnittlichen Kampagne und Opposition in der prognostizierten Wahl abschneiden wird. Eine solche Erwartung stellen wir im Folgenden, basierend auf jüngsten Landtagswahlergebnissen, dem erwarteten Niveau des Wirtschaftswachstums im Quartal vor der Wahl, sowie aufgrund weiterer struktureller Informationen, auf. Somit setzt unser Modell einen Erwartungswert, vor dessen Hintergrund das Abschneiden der Parteien bewertet werden kann.

Während wir durch das Aufstellen von Erwartungswerten zunächst zum Verständnis der Bundestagswahlen 2017 beitragen, argumentieren wir zudem, dass unser Modell zwei Beiträge zur wissenschaftlichen Literatur über Wahlprognosen leistet. Der erste besteht darin, dass wir, soweit uns bekannt ist, die erste Prognose für nationale Wahlen abgeben, die auf Daten der Landesebene beruht. ${ }^{2}$ Genauer: Wir verwenden neben anderen Kovariaten Resultate von Landtagswahlen, um damit ein Modell zur Prognostizierung der Bundestagswahlergebnisse aller Parteien in jedem der Bundesländer bei allen nationalen Wahlen seit 1961 zu schätzen. Anschließend rechnen wir den prognostizierten Stimmanteil auf Landesebene unter Berücksichtigung der Wahlbeteiligung in absolute Stimmen um und aggregieren diese auf die Bundesebene hoch. Schon allein dieser Test trägt dazu bei, eine empirische Frage zu klären, die in den Medien hierzulande häufig aufgeworfen wird: Wie präzise sagen Wahlergebnisse auf Landesebene künftige Wahlen auf nationaler Ebene voraus?

2 Einige Wissenschaftler haben bereits aus den gleichen Gründen, die auch wir anführen, für andere Staaten als Deutschland subnationale Daten verwendet, um Wahlergebnisse auf nationaler Ebene zu prognostizieren. Dies gilt vor allem für Präsidentschaftswahlen in den USA (beispielsweise Campbell 1992; Jerôme u. Jerôme-Speziar 2012; Linzer 2013), aber auch für Wahlen in Großbritannien (Rallings u. Thrasher_1999). Im Falle Deutschlands besteht jedoch sowohl der Vorteil von verstreut datierten Wahlen, als auch dem der kollektiven geografischen Abdeckung des gesamten Bundesgebietes. 
Zudem gibt es eine Reihe von Gründen, die eine Analyse der Landesebene zum Zweck der Prognostizierung der Bundestagswahl sinnvoll erscheinen lassen. Zunächst stattet uns ein solches Vorgehen mit mehr Beobachtungen aus, welche wiederum mehr Variablen in unseren Modellen stützen und dennoch das Verhältnis von Variablen zu Beobachtungen verringern. Damit vermindert sich die Gefahr einer Überanpassung des Modells an die Daten. Des Weiteren liefern mehr Beobachtungen auch mehr Informationen. Dies ist insbesondere dann der Fall, wenn die Wahlen über den Kalender verstreut stattfinden und deshalb Geschehnisse aufgreifen können, die zu einem Zeitpunkt nach der letzten Bundestagswahl stattgefunden haben. ${ }^{3}$ Sicherlich sind dazu auch Umfragedaten, wie etwa zur Parteiidentifikation (Kayser u. Leininger 2016) oder zur Popularität der Kanzlerin oder des Kanzlers (Norpoth u. Gschwend 2013) bis zu einem gewissen Grad in der Lage. Im Gegensatz zu flüchtigen Meinungsumfragen spiegeln die zusätzlich aus den Landtagswahlen gewonnenen Informationen jedoch das tatsächliche statt hypothetische Wahlverhalten von Befragten wider. Wie Umfrage- und Prognoseaggregatoren zeigen, ist der Prognosefehler eines Durchschnitts mehrerer Vorhersagen (in unserem Fall mehrerer Landesprognosen) oftmals kleiner als der die Abweichung der einzelnen Prognosen vom tatsächlichen Ergebnis (Graefe 2015). Dies gilt insbesondere dann, wenn Landtagswahlen zu verschiedenen Zeiten stattfinden und damit weniger anfällig für korrelierte Fehler sind.

Unser zweiter Beitrag zur Literatur besteht in der Anwendung eines Mehrebenenmodells, welches die Wahlergebnisse für jede Partei in jedem Bundesland vorhersagt. Unsere Entscheidung für ein solches Modell gründet in der Erkenntnis, dass ein simpleres Modell mit einer Prognose des Stimmanteils der scheidenden Regierung von geringem Interesse wäre, wenn diese, wie es im Jahr 2017 der Fall ist, eine Große Koalition ist. Wir sind allerdings nicht die Ersten, die Stimmanteile für die einzelnen Parteien in einer deutschen Wahl prognostizieren. So haben beispielsweise Jérôme et al. (2013) bereits ein SUR-Modell („Seemingly Unrelated Regression“) mit mehreren Schätzgleichungen angewendet. Unser Modell fügt dem jedoch den Vorzug hinzu, dass Schätzungen für mehrere Parteien in jeweils allen Bundesländern abgegeben werden.

Im vergangenen bundesdeutschen Wahlzyklus von 2013 rückten bis dato vernachlässigte Ansätze zur Prognose erstmals stärker in den Vordergrund. Norpoth und Gschwends (2013) wegweisendes Kanzlermodell wurde sowohl durch Strukturmodelle ergänzt, welche die deutsche Wirtschaftsleistung in Bezug zur Wirtschaftleistung der nächstgrößten Volkswirtschaften Großbritannien, Frankreich und Italien setzen (Kayser u. Leininger 2016), als auch durch Mehrfachgleichungsmodelle, die den Stimmanteil einer jeden Partei prognostizieren (Jérôme et al. 2013; Selb u. Munzert 2016), sowie weiteren Hybridformen von Prognose-

3 Als ein Beispiel hierfür kann die Nuklearkatastrophe in Fukushima am 11. März 2011 - mitten in der 17. Legislaturperiode - gennant werden. Sie hatte einen starken und, wie wir mittlerweile wissen, dauerhaften Ansteig der Popularität der Grünen in Baden-Württemberg zur Folge. Bündnis 90/Die Grünen erzielten bei der Landtagswahl 2011 das zweitbeste Stimmergebnis und stellen seitdem den Ministerpräsidenten. 
methoden (beispielsweise Graefe 2015, Küntzler 2017). Es ist zu hoffen, dass die aktuelle Wahlperiode ähnliche Innovationen hervorbringen wird.

\section{Das Modell}

Wir haben die Daten der Ergebnisse aller Bundes- und Landtagswahlen seit 1961 zusammengetragen. Damit erhalten wir einen Panel-Datensatz, in dem das Ergebnis einer Partei in einer Bundestagswahl in einem der 16 Bundesländer die Beobachtungseinheit bildet. Die Paneldaten sind unbalanciert, da in den vergangenen Jahren nicht alle Parteien zu allen Wahlen und in allen Bundesländern angetreten sind. ${ }^{4}$ Wir fokussieren uns daher auf die Parteien CDU/CSU, SPD, FDP, Bündnis 90/Die Grünen, Die Linke/PDS und eine Residualkategorie Sonstige. Um die Stimmanteile dieser Parteien vorherzusagen, schätzen wir ein lineares Mehrebenenmodell, welches gruppenspezifische Regressionskonstanten („,random intercepts“) für Staaten und Parteien beinhaltet. Diese modellieren wir so, dass wir Parteien als Subebene innerhalb der Länder betrachten.

Unser Modell setzt sich aus den folgenden Variablen zusammen: dem Stimmanteil, den eine Partei bei der vorigen Bundestagswahl erreicht hat, dem Stimmanteil, den sie in der vorangegangen Landtagswahl erhalten hat, einer Variable, die besagt, ob die Partei zum Zeitpunkt der fraglichen Wahl die Kanzlerin oder den Kanzler stellte, das bundesweite Wachstum des Bruttoinlandsprodukts im Quartal vor der Wahl ${ }^{5}$, die Interaktion dieser beiden Variablen, die Anzahl der Jahre, welche die Kanzlerin oder der Kanzler schon im Amt war, und deren Interaktion mit der Dummy-Variable der Kanzlerpartei.

Die Berücksichtigung des Stimmanteils einer Partei aus der vorherigen Bundestagswahl erlaubt es uns, eine Basiserwartung auf Grundlage des langfristigen Trends des Stimmanteils der Partei zu formulieren. Außerdem dient uns diese Variable als ein Maßstab zur Bewertung der Genauigkeit unseres Modells. Zusätzlich inkludieren wir weitere Variablen, welche dann effektiv Abweichungen von dem auf Basis des vorherigen Stimmanteils erwarteten Trends berücksichtigen. Die Inklusion einer zeitverzögerten Variablen in einem Random-Effects-Modell ist in klassischen Anwendungen nicht unproblematisch, da diese Variable mit dem Random Effect im Fehlerterm korreliert sein wird. Dies betrachten wir für unsere Zwecke als vernachlässigbar, da es uns nicht um die Genauigkeit der Schätzung von Regressionskoeffizienten und Standardfehlern geht. Unser Ziel ist es, die abhängige Variable möglichst akkurat vorherzusagen. Wesentliches Maß hierfür ist

4 Während CDU/CSU, SPD und FDP in nahezu jeder Bundes- und Landtagswahl in jedem einzelnen Bundesland angetreten sind, wurden Bündnis 90/Die Grünen sowie Die Linke (vorher: PDS) später gegründet. Die Partei Bündnis 90/Die Grünen existiert seit 1979 und trat seit 1980 zu allen bundesweiten und den meisten landesweiten Wahlen an. Die Linke, im Dezember 1989 als PDS gegründet, trat seitdem zu allen Bundestags- und zu Landtagswahlen in Ostdeutschland an. Nach dem Zusammenschluss mit der WASG und der Umbenennung in Die Linke nahm sie zudem auch an allen westdeutschen Landtagswahlen teil.

5 Einige Befunde deuten darauf hin, dass zu diesem Zeitpunkt aktuelle Schätzungen der Wirtschaftsleistung, möglicherweise aufgrund ihrer höheren Medienpräsenz im Vergleich zu nachträglich überarbeiteten Zahlen, die Wahlprognose verbessern können (Kayser u. Leininger 2015). Zeitund Datenbeschränkungen hindern uns allerdings daran, auf diese zurückzugreifen. 
die Genauigkeit der „Out-of-sample“-Prognosen, nicht die statistische Signifikanz der einzelnen Prädiktoren.

Tabelle 1: Zwei Mehrebenenmodelle zur Prognose der Stimmanteile der CDU/ CSU, SPD, FDP, Die Linke, Bündnis 90/Die Grünen sowie Sonstiger

\begin{tabular}{|c|c|c|}
\hline & $\begin{array}{c}1) \\
\text { Ungewichtet }\end{array}$ & $\begin{array}{c}(2) \\
\text { Gewichtet }\end{array}$ \\
\hline Stimmanteil $\mathrm{t}_{\mathrm{t}-1}$ & $\begin{array}{l}0,541^{* * *} \\
(0,0279)\end{array}$ & $\begin{array}{c}0,0995^{*} \\
(0,0434)\end{array}$ \\
\hline Stimmanteil in Landtagswahl & $\begin{array}{l}0,382^{* * *} \\
(0,0246)\end{array}$ & $\begin{array}{l}0,468^{* * * *} \\
(0,0736)\end{array}$ \\
\hline Kanzlerpartei & $\begin{array}{l}4,729 * * * \\
(0,681)\end{array}$ & $\begin{array}{l}8,695^{* * *} \\
(1,008)\end{array}$ \\
\hline BIP-Wachstum & $\begin{array}{l}-0,00999 \\
(0,0419)\end{array}$ & $\begin{array}{l}-0,0457 \\
(0,0269)\end{array}$ \\
\hline Kanzlerpartei $\times$ BIP-Wachstum & $\begin{array}{c}0,249^{* *} \\
(0,0937)\end{array}$ & $\begin{array}{l}0,554^{* *} \\
(0,185)\end{array}$ \\
\hline Jahre in der Regierung & $\begin{array}{c}0,0570 \\
(0,0347)\end{array}$ & $\begin{array}{c}0,105^{*} \\
(0,0453)\end{array}$ \\
\hline Kanzlerpartei $\times$ Jahre in der Regierung & $\begin{array}{l}-0,399 * * * \\
(0,0769)\end{array}$ & $\begin{array}{l}-0,682^{* * *} \\
(0,145)\end{array}$ \\
\hline Konstante & $\begin{array}{c}0,561 \\
(0,332)\end{array}$ & $\begin{array}{l}6,015^{* * *} \\
(0,988)\end{array}$ \\
\hline$\sigma$ Bundesland: Stimmanteil in Landtagswahl & $\begin{array}{l}4,31 \mathrm{e}-09 \\
(,)\end{array}$ & $\begin{array}{l}0,222^{* * *} \\
(0,0545)\end{array}$ \\
\hline$\sigma$ Bundesland: Konstante & $\begin{array}{l}6,29 \mathrm{e}-08 \\
(,)\end{array}$ & $\begin{array}{l}2,749^{* *} \\
(1,074)\end{array}$ \\
\hline$\sigma$ Partei $\times$ Bundesland: Konstante & $\begin{array}{l}0,393 \\
(,)\end{array}$ & $\begin{array}{l}6,349^{* * *} \\
(0,938)\end{array}$ \\
\hline$\sigma$ Residuale & $\begin{array}{l}3,828 \\
(,)\end{array}$ & $\begin{array}{l}2,401 * * * \\
(0,261)\end{array}$ \\
\hline$N$ & 872 & 872 \\
\hline
\end{tabular}

Anmerkung: Modell (2) ist so gewichtet, dass Landtagswahlen, die zeitlich näher an einer Bundestagswahl liegen, mehr Einfluss haben. Standardfehler sind in Klammern. $\sigma$ bezeichnet die Varianzen der Random Intercepts und Coefficients. ${ }^{*}(p<0.05),{ }^{* *}(p<0.01),{ }^{* * *}(p<0.001)$.

Quelle: eigene Berechnung.

Zusätzlich beziehen wir auch den Stimmanteil einer Partei bei den vorangegangenen Landtagswahlen in unser Modell mit ein. Diesen modellieren wir als „random slope“, um der Möglichkeit Rechnung zu tragen, dass die Korrelation zwischen Landtags- und Bundestagswahlen zwischen den Ländern variiert. 
Landesspezifische Fragen spielen zwar während der Wahlkämpfe eine große Rolle - so sind häufig erhebliche Unterschiede zwischen den Ergebnissen einer Partei auf Landes- und auf Bundesebene auszumachen - allerdings verringern sich diese, sobald man, wie hier geschehen, die bundesweiten Stimmanteile in jedem Bundesland prognostiziert. Nichtsdestotrotz gelten Stimmanteile in Landtagswahlen als ein Thermometer für die Popularität der Bundesregierung und der Oppositionsparteien auf Bundesebene. Dies gilt umso mehr, wenn eine Bundestagswahl unmittelbar bevorsteht. Aus diesem Grund kann es sinnvoll sein, die Daten auf Basis des zeitlichen Abstands der Landtagswahl zur Bundestagswahl zu gewichten. Wir schätzen zwei minimal unterschiedliche Varianten unseres Modells. In der zweiten Variante gewichten wir Beobachtungen stärker, die eine besonders nah vor einer Bundestagswahl liegende Landtagswahl repräsentieren.

Wir kodieren zudem eine Dummy-Variable, die angibt, ob die amtierende Kanzlerin oder der amtierende Kanzler der jeweiligen Partei angehört. Für die unterschiedlichen Wahlperioden ist die Variable folglich also entweder für die CDU/ CSU oder die SPD gleich eins. Weiterhin inkludieren wir die saisonbereinigte Wachstumsrate des BIP für das der Wahl vorangegangene Quartal im Vergleich zu demselben Quartal des vorigen Jahres. ${ }^{6}$ In der Economic-Voting-Literatur gilt das Wirtschaftswachstum als wichtigste Variable. Es wurde schon zuvor erfolgreich zur Prognose bundesdeutscher Wahlen herangezogen (beispielsweise als „benchmarked growth “, siehe Kayser u. Leininger 2016). Die Wachstumsrate interagieren wir zudem mit der Dummy-Variable der Kanzlerpartei, da die Verantwortung für den Zustand der Wirtschaft in erster Linie der Partei des Regierungschefs zugeschrieben wird (Duch et al. 2015). Weiterhin beziehen wir die Anzahl der Jahre mit ein, welche die Kanzlerin oder der Kanzler zum Zeitpunkt der Wahl bereits das Amt bekleidet hat, um dadurch „Cost of Ruling“-Effekte erfassen zu können, die wir mit der Dummy-Variable der Kanzlerpartei verbinden können.

Es sei an dieser Stelle angemerkt, dass es sich bei unserem Modell um ein gänzlich strukturelles Modell handelt, das sich nicht auf umfragebasierte Variablen stützt. Eine kleine Ausnahme müssen wir lediglich bezüglich des Stimmanteils bei den Landtagswahlen machen: Aufgrund der unterschiedlichen Dauer der Legislaturperioden auf Bundes- und Landesebene ${ }^{7}$ und der vier vorgezogenen Bundestagswahlen ${ }^{8}$ findet in vereinzelten Fällen in wenigen Bundesländern keine Landtagswahl zwischen zwei Bundestagswahlen statt. Falls entsprechende Daten verfügbar sind, rechnen wir für diesen Fall mit Umfrageresultaten, die auf Landesebene mindestens sechs Monate vor der jeweiligen Bundestagswahl erhoben wurden.

6 Die Daten des Wirtschaftswachstums von 1961 bis 2016 sind aus der Main Economic Indicators (MEI) database der OECD. Prognosen für 2017 wurden von der Unternehmensberatung Trading Economics bezogen.

7 Die Dauer der Legislaturperiode beträgt auf Bundesebene vier Jahre, während es auf Landesebene in nahezu allen Bundesländern fünf Jahre sind.

8 Die Wiederwahl Willy Brandts von 1972, die aufgrund eines verlorenen Misstrauensvotums vorgezogen wurde; die Wahl von Helmut Kohl zum Kanzler 1983, nachdem Helmut Schmidt durch ein Misstrauensvotum seines Amtes enthoben wurde; die Neuwahlen nach der Wiedervereinigung 1990; die vorgezogenen Wahlen von 2005, denen ein verlorenes Misstrauensvotum Gerhard Schröders vorausging. 


\section{Unsere Prognose}

In einem linearen Mehrebenenmodell regressieren wir den Stimmanteil der fünf Parteien CDU/CSU, SPD, FDP, Die Linke/PDS, Bündnis 90/Die Grünen sowie der Residualkategorie Sonstige auf unsere erklärenden Variablen. Mittels der so geschätzten Gleichung können wir unter Einsetzung aktueller Werte für die erklärenden Variablen unsere Prognose für 2017 berechnen. Wir schätzen zwei Modelle, ein ungewichtetes und ein gewichtetes. Beide sind in Tabelle 1 dargestellt. Das zweite Modell gewichtet Landtagswahlen, die zeitlich näher an den Bundestagswahlen liegen höher, um dadurch spät stattfindende Ereignisse stärker in die Prognose einbeziehen zu können.

Alle Koeffizienten tragen das erwartete Vorzeichen. Es existiert eine starke positive Korrelation im Stimmanteil einer Partei über die Zeit hinweg. Dasselbe gilt für solche Landtagswahlen, die zwischen zwei Bundestagswahlen liegen. Ein starker positiver Koeffizient auf der Dummy-Variable der Kanzlerpartei spiegelt lediglich die Tatsache wider, dass der Kanzler oder die Kanzlerin in Deutschland stets von einer der beiden großen Parteien gestellt wird. Zusätzlich könnte dies aber auch einen Amstinhaberbonus darstellen. Der Koeffizient des BIP-Wachstums hängt vom Status einer Partei ab: Wie erwartet, gibt es keine Verbindung zwischen Wirtschaftswachstum und dem Stimmanteil einer Partei, wenn diese nicht die Bundesregierung anführt. Wenn eine Partei jedoch an der Spitze der Regierung steht, dann ist der erwartete positive Zusammenhang gegeben. Die Länge der Amtszeit eines amtierenden Kanzlers oder einer Kanzlerin hat im Großen und Ganzen keine Vorhersagekraft für den Stimmanteil einer Oppositionspartei. Allerdings ist der Koeffizient der Jahre im Amt signifikant negativ für die Partei des Amtsinhabers, was den erwarteten „Cost of Ruling“-Effekt darstellt.

Tabelle 2: Prognosen für die fünf großen Parteien und eine Residualkategorie Sonstige (beinhaltet die $A f D$ ) - basierend auf den Modellen ohne und mit Gewichtung

\begin{tabular}{lcccc}
\hline & $\begin{array}{c}\text { Prognose } \\
\text { (ohne Gewichtung) }\end{array}$ & $\begin{array}{c}\text { Prognose } \\
\text { (mit Gewichtung) }\end{array}$ & $\begin{array}{c}\text { Prä-Schulz- } \\
\text { Umfrage }\end{array}$ & $\begin{array}{c}\text { Umfragen } \\
\text { Mai 2017 }\end{array}$ \\
\hline CDU/CSU & $36,8[34,2 ; 39,9]$ & $35,1[31,6 ; 39,0]$ & 36 & 37,4 \\
SPD & $24,4[22,4 ; 26,9]$ & $26,1[22,5 ; 29,7]$ & 21 & 27,1 \\
Die Linke & $8,5[5,7 ; 11,0]$ & $9,2[6,0 ; 12,5]$ & 9 & 8,4 \\
Bündnis 90/Die Grünen & $10,4[7,9 ; 12,7]$ & $10,5[6,6 ; 13,8]$ & 10 & 7,0 \\
FDP & $6,6[4,2 ; 8,8]$ & $8,7[5,0 ; 12,0]$ & 6 & 7,9 \\
Sonstige & $13,2[10,8 ; 15,7]$ & $10,5[7,1 ; 14,1]$ & 18 & 12,2 \\
\hline
\end{tabular}

Anmerkungen: Simulationsbasierte $95 \%$-Prognoseintervalle sind in eckigen Klammern. Spalten 4 und 5 zeigen jeweils durchschnittliche Werte von Umfragen zur Zeit unseres Entwurfs (31. Mai 2017) und die letzte Umfrage der Forschungsgruppe Wahlen, bevor die SPD Martin Schulz als ihren Kanzlerkandidaten verkündet hat (14. Januar 2017).

Quelle: eigene Berechnung.

Fügen wir die Werte von 2017 für unsere erklärenden Variablen in die Gleichung ein, so erhalten wir Prognosen für alle Parteien und für alle 16 Bundesländer. 
Diese Länderdaten aggregieren wir dann auf Bundesebene hoch, um zu einer Schätzung der bundesweiten Stimmenverteilung zu gelangen, welche letztlich das Kräftegleichgewicht im Bundestag bestimmt. Um Unterschiede in den Größen der Wählerschaften und den Höhen der Wahlbeteiligungen zwischen den Ländern zu berücksichtigen, übersetzen wir den Stimmanteil einer Partei in einem Bundesland in absolute Stimmenanzahlen. Dies geschieht, indem wir eine vom Bundeswahlleiter veröffentlichte aktuelle Schätzung der Größe der jeweiligen Wählerschaft mit den erwarteten Stimmanteilen und der erwarteten Wahlbeteiligung in jedem Bundesland multiplizieren. Letzteres wird in einem separaten Modell geschätzt. ${ }^{9}$ Anschließend addieren wir die absoluten Stimmenanzahlen der Parteien über alle Bundesländer hinweg auf und rechnen diese dann wieder in Prozentwerte um. Damit gelangen wir zu einer Schätzung des bundesweiten Stimmanteils für jede Partei. Um die Unsicherheit einzubeziehen, die mit den Schätzungen der Stimmanteile und der Wahlbeteiligung einhergeht, simulieren wir eine Vielzahl von Vorhersagen für beide Modelle, fügen diese zusammen und aggregieren sie über die simulierten Daten, um 95-prozentige Prognoseintervalle bereitzustellen.

Tabelle 2 bildet unsere Prognosen ab. In beiden Modellen behält die CDU/CSU die Mehrheit. Jedoch verliert sie im Vergleich zu ihrem Abschneiden bei den Wahlen 2013 mindestens 5 Prozentpunkte. ${ }^{10}$ Basierend auf unserem Modell erwarten wir weiterhin, dass die SPD mit etwa 25 bis 27 Prozent der Stimmen abschneidet, was etwa ihrem Ergebnis der vorherigen Wahl entspricht. Dies stellt außerdem eine Verbesserung gegenüber solchen Umfragewerten dar, die vor der Ernennung von Martin Schulz zum Kanzlerkandidaten erzielt wurden. Zugleich legt unsere Prognose den Schluss nahe, dass aktuelle Umfragen ${ }^{11}$ die Unterstützung für die SPD überbewerten. Die Prognosen für Die Linke und Bündnis 90/Die Grünen sind in beiden Modellen verhältnismäßig stabil. Im gewichteten Modell erwarten wir ein stärkeres Abschneiden der FDP und ein schlechteres Resultat der Residualkategorie Sonstige. Das ist insofern nicht verwunderlich, als dass die Ergebnisse dieser beiden in vergangenen Bundestagswahlen recht volatil waren.

Als Haupttest der Validität unseres Modells werden die Resultate der Bundestagswahl 2017 dienen. Die Prognosevalidität eines solchen Modells, wie es hier zur Anwendung kommt, beruht im Kern auf dessen Fähigkeit, Ergebnisse von Wahlen vorherzusagen, die nicht selbst Teil dessen Stichprobe sind. Als wir unser Modell Anfang 2017 anfertigten, führten wir zur Überprüfung der Vorhersagekraft unseres Modells daher synthetische „Out-of-sample“-Prognosen durch.

Dazu schätzen wir unser Modell mit einem reduzierten Satz von Wahlen bis ausschließlich der Bundestagswahl von 1998. Anschließend wiederholen wir dies für alle weiteren Wahlen bis 2013. Für diese fünf Perioden vergleichen wir unsere Prognosen dann mit den tatsächlichen Resultaten. Den Prognosefehler, also die Abweichung zwischen Vorhersage und tatsächlichem Wahlergebnis, fassen wir als

9 Wir verwenden ein lineares Mehrebenenmodell unter Einbeziehung früherer Wahlbeteiligungen, landesspezifischer Entwicklungen und fester Effekte, um das Niveau der Wahlbeteiligung für ein Bundesland im Jahr 2017 zu prognostizieren.

10 Die Ergebnisse der Bundestagswahl 2013 lauteten wie folgt: CDU/CSU: 41,5 \%, SPD: 25,7 \%, Die Linke: 8,6 \%, Bündnis 90/Die Grünen: 8,4 \%, FDP: 4,8 \%, Andere: 10,9\%.

11 Zum Zeitpunkt Mai 2017. 
„Mean Absolute Error“ (MAE) und „Root Mean Squared Error“ (RMSE) innerhalb der einzelnen Parteien und über die Parteien hinweg zusammen (siehe Tabelle 3). Dies zeigt uns, welchen Genauigkeitsgrad wir in etwa für unsere Prognose der Bundestagswahl 2017 erwarten können. Außerdem vergleichen wir unser Regressionsmodell mit weitaus simpleren Prognosen. Die erste davon bedient sich als Basis ihrer Vorhersage des Stimmanteils, den eine Partei in der vorangegangenen Wahl erlangt hat, während bei der zweiten der Durchschnitt der Resultate einer Partei in allen bisherigen Bundestagswahlen seit 1961 herangezogen wird. Es wird ersichtlich, dass unser Modell deutlich besser abschneidet als diese naiven ,Benchmarks‘. Dies gilt für alle Fälle bis auf eine einzige Partei und einen speziellen Benchmark: So lässt sich das Ergebnis von Bündnis 90/Die Grünen etwas besser durch ihre zuvor erlangten Resultate vorhersagen als durch unser Modell. Dem aufmerksamen Leser könnten zudem die Vorzüge des Aggregierens von Länderdaten hoch zur Bundesebene aufgefallen sein: Für alle Prognosemethoden gilt, dass die Fehler auf Bundesebene durchweg erheblich kleiner sind als für Prognosen auf Landesebene.

Tabelle 3: Parteispezifische und Gesamtprognosefehler - basierend auf „Out-ofsample“-Prognosen der Bundestagswahlen von 1998-2013

\begin{tabular}{|c|c|c|c|c|c|c|c|c|}
\hline \multirow[b]{2}{*}{ Partei } & \multicolumn{2}{|c|}{$\begin{array}{c}\text { Prognose } \\
\text { (ungewichtet) }\end{array}$} & \multicolumn{2}{|c|}{$\begin{array}{l}\text { Prognose } \\
\text { (gewichtet) }\end{array}$} & \multicolumn{2}{|c|}{$\begin{array}{l}\text { Vorheriger } \\
\text { Stimmanteil }\end{array}$} & \multicolumn{2}{|c|}{$\begin{array}{l}\text { Durchschnitt- } \\
\text { licher Stimmantei }\end{array}$} \\
\hline & MAE & RMS & MAE & RMS & MAE & RMS & MAE & RMS \\
\hline \multicolumn{9}{|l|}{ Bundesebene } \\
\hline $\mathrm{CDU} / \mathrm{CSU}$ & 4,2 & 5,0 & 3,4 & 3,8 & 4,4 & 5,0 & 7,2 & 8,0 \\
\hline SPD & 3,4 & 5,2 & 2,8 & 3,6 & 5,0 & 6,0 & 7,2 & 9,4 \\
\hline Die Linke/PDS & 2,2 & 2,7 & 1,6 & 1,9 & 2,6 & 3,0 & 3,2 & 4,0 \\
\hline Bündnis 90/Die Grünen & 1,6 & 1,9 & 1,8 & 1,9 & 1,5 & 1,8 & 2,6 & 2,8 \\
\hline FDP & 2,8 & 3,8 & 2,5 & 2,8 & 3,8 & 5,0 & 3,1 & 3,6 \\
\hline Sonstige & 2,0 & 2,3 & 1,5 & 2,2 & 2,6 & 2,9 & 2,9 & 3,9 \\
\hline Insgesamt & 2,7 & 3,7 & 2,3 & 2,8 & 3,3 & 4,2 & 4,4 & 5,8 \\
\hline \multicolumn{9}{|l|}{ Länderebene } \\
\hline Insgesamt & 3,6 & 4,8 & 2,9 & 3,9 & 3,6 & 4,9 & 4,6 & 6,1 \\
\hline
\end{tabular}

Anmerkungen: Der mittlere absolute Prognosefehler („Mean Absolute Error“ [MAE]) und der „Root Mean Squared Error“ (RMSE) des ungewichteten und des gewichteten Modells werden mit zwei „,naiven" Prognosen verglichen: Die eine prognostiziert den Stimmanteil auf Basis des bei der letzten Wahl erreichten Stimmanteils, die zweite sagt diesen unter Berücksichtigung aller bislang erreichten Stimmanteile voraus.

Quelle: eigene Berechnungen.

Diese Prognosen stellen zum jetzigen Zeitpunkt die aus unserer Sicht bestmöglich theoretisch wie empirisch abgesicherten Vorhersagen des Ausgangs der Bundestagswahl 2017 dar. Womöglich wird unsere Prognose, welche wir etwa vier Monate vor der Wahl abgeben und nicht mehr anpassen werden, weiter von dem tatsächlichen Wahlergebnis abweichen als Umfragen, die kurz vor dem Wahltag durchgeführt werden. Nichtsdestotrotz bietet unsere Prognose auch dann einen echten Mehrwert gegenüber Umfragen. Mittels unseres theoretisch motivierten 
Prognosemodells auf Grundlage des Zusammenhangs zwischen vorangegangenen Wahlen und politischer wie ökonomischer Faktoren sagen wir im Grunde voraus, wie ein durchschnittlicher Kandidat mit einer durchschnittlichen Kampagne und Opposition in der prognostizierten Wahl abschneiden wird. Somit setzt unser Modell einen Erwartungswert, vor dessen Hintergrund das tatsächliche Abschneiden der Parteien bewertet und die Wahl eingeordnet werden kann - sowohl für die Bundes- als auch für die Landesebene.

\section{Literatur}

Blumenthal, Mark. 2014. Polls, forecasts, and aggregators. Political Science and Politics 47(2): 297-300. DOI: 10.1017/S1049096514000055.

Campbell, James E. 1992. Forecasting the Presidential Vote in the States. American Journal of Political Science 36(2): 386-407. DOI: 10.2307/2111483.

Duch, Raymond, Wojtek Przepiorka, und Randolph Stevenson. 2015. Responsibility attribution for collective decision makers. American Journal of Political Science 59(2): 372389. DOI: 10.1111 /ajps.12140.

Erikson, Robert S., und Christopher Wlezien. 2012. The timeline of presidential elections: How campaigns do (and do not) matter. Chicago, IL: University of Chicago Press.

Erikson, Robert S., und Christopher Wlezien. 2014. Forecasting US presidential elections using economic and noneconomic fundamentals. Political Science and Politics 47(2): 313-316. DOI: 10.1017/S1049096514000092.

Graefe, Andreas. 2015. German election forecasting: Comparing and combining methods for 2013. German Politics 24(2): 195-204. DOI: 10.1080/09644008.2015.1024240.

Jerôme, Bruno, und Véronique Jerôme-Speziari. 2012. Forecasting the 2012 US presidential election: Lessons from a state-by-state political economy model. Political Science and Politics 45(4): 663-668. DOI: 10.1017/S1049096512000972.

Jérôme, Bruno, Véronique Jérôme-Speziari, und Michael S. Lewis-Beck. 2013. A political-economy forecast for the 2013 German elections: who to rule with Angela Merkel? Political Science and Politics 46(3): 479-480. DOI: 10.1017/S1049096513000814.

Kayser, Mark A., und Arndt Leininger. 2015. Vintage errors: do real-time economic data improve election forecasts? Research and Politics 2(3). DOI: 10.1177/2053168015589624.

Kayser, Mark A. und Arndt Leininger. 2016. A Predictive Test of Voters' Economic Benchmarking: The 2013 German Bundestag Election. German Politics 25(1): 106-130. DOI: $10.1080 / 09644008.2015 .1129531$.

Küntzler, Theresa. 2017. Using Data Combination of Fundamental Variable-Based Forecasts and Poll-Based Forecasts to Predict the 2013 German Election. German Politics: 1-19, published online first. DOI: 10.1080/09644008.2017.1280781.

Linzer, Drew A. 2013. Dynamic Bayesian forecasting of presidential elections in the States. Journal of the American Statistical Association 108(501): 124-134. DOI: 10.1080/ 01621459.2012.737735.

Norpoth, Helmut, und Thomas Gschwend. 2013. Chancellor model picks Merkel in 2013 German election. Political Science and Politics 46(3): 481-482. DOI: $10.1017 /$ S1049096513000802. 
Rallings, Colin, und Michael Thrasher. 1999. Local votes, national forecasts-using local government by-elections in Britain to estimate party support. International Journal of Forecasting 15(2): 153-162. DOI: 10.1016/S0169-2070(98)00062-4.

Selb, Peter, und Simon Munzert. 2016. Forecasting the 2013 German Bundestag Election Using Many Polls and Historical Election Results. German Politics 25(1): 73-83. DOI: 10.1080/09644008.2015.1121454.

\section{Autorenangaben}

Arndt Leininger, Ph.D.

Universität Mainz

Jakob Welder-Weg 12

55128 Mainz

E-Mail: leininger@politik.uni-mainz.de

Prof. Mark A. Kayser, Ph.D.

Professor für Angewandte Methoden und Vergleichende Politikwissenschaft Hertie School of Governance

Friedrichstraße 180

10117 Berlin

E-Mail: kayser@hertie-school.org 


\title{
Zweitstimme.org. Ein strukturell-dynamisches Vorhersagemodell für Bundestagswahlen
}

\author{
Simon Munzert, Lukas Stötzer, Thomas Gschwend, Marcel Neunhoeffer und \\ Sebastian Sternberg
}

Zweitstimme.org. A structural-dynamic forecasting model for German federal elections Abstract: We present results of an ex-ante forecast of party-specific vote shares at the German Federal Election 2017. To that end, we combine data from published trial heat polls with structural information. The model takes care of the multi-party nature of the setting and allows making statements about the probability of certain events, such as the plurality of votes for a party or the majority for coalition options in parliament. The forecasts of our model are continuously being updated on the platform zweitstimme.org. The value of our approach goes beyond the realms of academia: We equip journalists, political pundits, and ordinary citizens with information that can help make sense of the parties' latent support and ultimately make voting decisions better informed.

Keywords: Bundestag election, forecast, polls, Bayesian modeling, probability

Schlagworte: Bundestagswahl, Vorhersage, Umfragen, Bayesianische Modellierung, Wahrscheinlichkeit

\section{Einleitung ${ }^{1}$}

Wer wird stärkste Partei im nächsten Bundestag und stellt möglicherweise den nächsten Kanzler? Erreicht eine Ampelkoalition oder eine Koalition aus SPD, B'90/Die Grünen und der Linken eine rechnerische Mehrheit? Schafft es die FDP wieder in den Bundestag? In der medialen Berichterstattung zur Bundestagswahl 2017 nehmen Fragen wie diese einen wichtigen Platz ein. Dabei dienen insbesondere nahezu täglich veröffentlichte Befragungen als Evidenz. Allerdings stellen sie weder eine besonders zuverlässige Grundlage dar, um den Monate in der Zukunft liegenden Wahlausgang vorherzusagen, noch, um die Unsicherheit einzuordnen, die mit der Beantwortung der eben genannten Fragen einhergeht.

In diesem Beitrag präsentieren wir einen neuen Ansatz, um sowohl Zweitstimmenergebnisse verschiedener Parteien als auch entsprechende Koalitionsoptionen für die kommende Bundestagswahl 2017 vorherzusagen. Dabei stehen drei Ziele im Vordergrund: (1) Frühzeitige und möglichst präzise Vorhersage des Bundes-

1 Für hilfreiche Kommentare danken wir Marc Debus, Helmut Norpoth, Kai-Uwe Schnapp und Steffen Zittlau sowie zwei anonymen Gutachtern. Den Editoren danken wir für die schnelle Bearbeitung unserer Einreichung, die eine Veröffentlichung der Vorhersage noch vor der Wahl möglich machte. Darüber hinaus bedanken wir uns herzlich für die Überlassung von Daten, insbesondere hinsichtlich der Sonntagsfrage bei Jochen Groß, Marcel Noack, Gertrud Petrig (Institut für Demoskopie Allensbach) sowie Rainer Schnell, und bei Christel Selzer für ihre redaktionelle Arbeit am Manuskript. 
tagswahlergebnisses und daraus ableitbarer Koalitions-Konstellationen, (2) Aktualisierung der Vorhersage auf Basis neu veröffentlichter Umfragen bis zur Bundestagswahl und (3) Berechnung und Kommunikation realistischer Unsicherheit sowie ableitbarer Wahrscheinlichkeiten für bestimmte Ereignisse, die mit den veröffentlichten Vorhersagen verbunden sind.

Bisherige Methoden zur Vorhersage von Bundestagswahlen basierten entweder auf strukturellen Modellen, die auf empirischen Regelmäßigkeiten bei bisherigen Bundestagswahlen beruhen, etwa dem robusten Zusammenhang zwischen Kanzlerpopularität und dem Stimmanteil der Koalitionsparteien - (Gschwend u. Norpoth 2000, 2001, 2017; Kayser u. Leininger 2016; Leininger u. Kayser 2017) oder auf Aggregationsmodellen, die Informationen aus veröffentlichten Umfragen kombinieren (Walther 2015; Selb u. Munzert 2016). Während rein umfragebasierte Modelle Informationen bis kurz vor dem Wahltag inkorporieren können, sind sie fehleranfällig für umfragespezifische Verzerrungen. Strukturelle Modelle erweisen sich dagegen trotz ihrer Datensparsamkeit als erstaunlich treffsicher (Gelman u. King 1993; Gschwend u. Norpoth 2005; Norpoth u. Gschwend 2003). Allerdings sind rein strukturelle Modelle meist nicht flexibel genug, um auf kurzfristige Neujustierungen im Parteiensystem oder der politischen Stimmung reagieren zu können und sind deswegen in Hinblick auf die vorhergesagten Größen nicht immer zielführend. So lassen viele der für die Bundestagswahlen vorgestellten Modelle lediglich Vorhersagen für den Anteil der Koalitionsparteien zu.

Kern unserer Strategie ist deshalb die Entwicklung eines kombinierten Modells, das die Stärken von strukturellen Modellen und Aggregationsmodellen in sich vereint. Dafür entwickeln wir ein strukturelles Modell, benutzen jedoch auch veröffentlichte Werte der sogenannten „Sonntagsfrage“ im Zeitverlauf, um die Zweitstimmenanteile von Parteien und mögliche Koalitionen vorherzusagen. Diese Kombination von strukturellen Modellen und Aggregationsmodellen von Umfragedaten zur Wahlvorhersage - wohlgemerkt ohne eigene Umfragen in Auftrag zu geben - ist für die Bundesrepublik ein Novum. Eine ähnliche Strategie wurde auf nationaler Ebene bisher nur zur Vorhersage des kombinierten Stimmenanteils der Regierungskoalition angewendet (Küntzler 2017). ${ }^{2}$

Während alle Informationen, die in den strukturellen Teil unseres Modells eingehen, bereits für die Prognose verfügbar sind, wird unser Modell immer dann aktualisierte Vorhersagen für den Wahlausgang der kommenden Bundestagswahl liefern, wenn neue Umfrageergebnisse zur "Sonntagsfrage“ veröffentlicht werden. Für die kommende Bundestagswahl am 24. September 2017 liefert unser Modell basierend auf Daten, die Stand 31. Mai 2017 verfügbar sind, folgende Vorhersage (mit $90 \%$-Kredibilitätsintervallen in Klammern): CDU/CSU 36,0 Prozent [28,3\%; 44,4 \%], SPD 26,4 Prozent [19,5\%; 34,7 \%], FDP 7,6 Prozent [4,9 \%; 11,4 \%], B'90/Die Grünen 7,2 Prozent [5,0 \%; 10,5 \%], Die Linke 8,1 Prozent $[5,8 \% ; 12,2 \%]$, und AfD 8,2 Prozent $[5,6 \% ; 11,9 \%]$.

2 Für einen Kombinationsansatz zur Vorhersage von Wahlergebnissen auf Wahlkreisebene außerdem Munzert (2016). 


\section{Ein neuer Vorhersageansatz}

Das hier vorgeschlagene Modell ist ein Hybrid aus einer strukturellen Komponente, das historische Zusammenhänge zwischen Fundamentalvariablen und Wahlanteilen einzelner Parteien auswertet, und einer dynamischen Komponente, die neue Information aus Umfragen vom Start der ersten Prognose bis zum Tag vor der Bundestagswahl in aktualisierte Vorhersagen einrechnet. In diesem Abschnitt stellen wir zunächst die Modellkomponente vor, die Vorhersagen auf Basis historischer Informationen generiert.

\subsection{Das strukturelle Modell}

Das strukturelle Modell erfüllt drei wesentliche Funktionen: Erstens erlaubt es uns eine frühzeitige Vorhersage des Wahlausgangs, weil die in das Modell eingehenden Prädiktoren bereits 200 Tage vor der Wahl feststehen. Dadurch kann zweitens die historische Vorhersage als Anker für das dynamische, umfragebasierte Modell genutzt werden, wie wir es im nächsten Abschnitt noch ausführlicher erläutern. Drittens erlaubt es die Auswertung historischer Beziehungen zwischen Wahlergebnissen und Strukturvariablen - das Modell lernt also aus den vergangenen 18 Bundestagswahlen seit 1949. Das ist insbesondere auch für eine realistische Einschätzung der Unsicherheit relevant, die mit den veröffentlichten Vorhersagen einhergeht, da die Schätzunsicherheit bei der geringen Zahl der Fälle doch beträchtlich ist.

Wir beginnen mit der Modellierung des Wahlanteils $y_{p t}$ einer Partei $p$ zum Zeitpunkt der Wahl $t$. Die Anzahl der modellierten Anteile der Parteien $p=1,2, \ldots, P$ variiert über Wahlen hinweg. Während beispielsweise bis zur Wahl 1979 lediglich die Anteile von CDU/CSU, SPD, FDP und Anderen (kombinierter Anteil der übrigen Parteien) modelliert werden, kommen ab 1980 der Anteil für B'90/Die Grünen, ab 1990 der Anteil für PDS/Die Linke und ab 2013 der AfD-Wähleranteil hinzu. Ziel ist es schließlich, von den Wahlen $w=1,2, \ldots, W-1$ auf den Ausgang der kommenden Wahl $W$ zu extrapolieren. Wir nehmen für $y_{p w}$ eine Normalverteilung mit Mittelwert $\mu_{p w}$ und Varianz $\sigma^{2}$ an,

$$
y_{p w} \sim N\left(\mu_{p w}, \sigma^{2}\right),
$$

wobei $\mu_{p w}$ als eine lineare Funktion von Kovariaten modelliert wird:

$$
\mu_{p w}=\beta_{w}^{0}+\sum_{k} \beta_{w}^{k} x_{p w}^{k} .
$$

Es besteht kein Konsens darüber, welche Variablenkombination in einem strukturellen Modell verwendet werden sollte, um die Stimmenanteile von Parteien bei Bundestagswahlen vorherzusagen. Die Vielfalt der Modelle und Prognosen allein schon in diesem Forum gibt bereits Aufschluss über die Unsicherheit, wie diese konkret modelliert werden sollten. Lauderdale u. Linzer (2015) bezeichnen letzteres als Spezifikationsunsicherheit. Eng damit verbunden ist das Bias-VarianzDilemma (Hastie et al. 2009), das, übertragen auf diese Anwendung, die Herausforderung beschreibt, Variablen zu identifizieren, die vergangene Wahlausgänge 
gut vorhersagen, sich jedoch auch für zukünftige Wahlen als prädiktiv erweisen. Wir begegnen diesem Problem mit folgender Strategie: Zunächst erstellen wir einen Datensatz mit einer Vielzahl plausibler Prädiktoren ${ }^{3}$. Auf Basis dieses Variablensamples generieren wir alle möglichen Kombinationen von 1 bis $K$ Prädiktoren (Gesamtzahl möglicher Kombinationen: 1023), berechnen auf Basis vergangener Wahlergebnisse lineare Modelle zur Erklärung parteispezifischer Wahlanteile und messen für jedes dieser Modelle die Varianzaufklärung. Dabei zeigen sich zwei Dinge: Erstens nimmt der relative Gewinn in der Varianzaufklärung für Modelle mit mehr als drei Prädiktoren deutlich ab. Gleichzeitig zeigen sich drei Variablen als konsistent robust, d. h. finden sich häufig im jeweils vorhersagestärksten Modell für eine festgelegte Zahl an Prädiktoren wieder. Diese robusten Faktoren sind (1) der Wahlanteil bei der zurückliegenden Bundestagswahl (für erstmals antretende Parteien mit 0 definiert), (2) der Mittelwert der in den Sonntagsfragen 230-200 Tage vor der Wahl veröffentlichten, parteispezifischen Anteile, und (3) ein Indikator für die Kanzlerpartei. Wir beschränken uns deshalb auf diese drei Variablen. Gleichzeitig lassen sich diese auch theoretisch sinnvoll motivieren und bieten so die Gelegenheit, etablierte Theorien zum Wahlverhalten über den Vorhersageansatz implizit zu testen. ${ }^{4}$

Wahlen finden nicht in einem politischen Vakuum statt. Wähler entwickeln langfristige Bindungen zu Parteien (Campbell et al. 1960). Die Verteilung der Parteibindungen in der Wählerschaft erlaubt es bereits Erwartungen zu bilden, welchen Stimmenanteil eine Partei unter „normalen Umständen“ erhalten sollte (Converse 1966; Falter u. Rattinger 1983). Wir operationalisieren den Wählerrückhalt, auf den Parteien unter normalen Umständen bauen können, als den errungenen Stimmenanteil einer Partei bei der letzten Bundestagswahl. Dieser Wert ist im Normalfall bereits vier Jahre vor einer Bundestagswahl verfügbar und dient deshalb als längerfristig wirksamer Prädiktor. ${ }^{5}$ Panel (a) in Abbildung 1 zeigt den

3 Dazu gehören Wahlanteil bei der zurückliegenden Wahl, mittlerer Wahlanteil bei den letzten drei zurückliegenden Wahlen, mittlere Zustimmung in Befragungen 230 - 200 Tage vor der Wahl, Abschneiden bei Landtagswahlen in der zurückliegenden Legislaturperiode, Indikatoren für den Status der Partei (Kanzlerpartei, große Partei [SPD oder CDU/CSU], Regierungspartei, im Parlament vertretene Partei), Arbeitslosigkeit ein Jahr vor der Wahl und Veränderung der Arbeitslosigkeit ein Jahr vor der Wahl.

4 Eine Alternative zu dieser „Ad-Hoc-Lösung“ zur Variablenauswahl wäre, wie von Lauderdale u. Linzer (2015) vorgeschlagen, die Implementation einer Regularisierung. Hierbei verhält man sich zunächst agnostisch gegenüber den auszuwählenden Variablen und nimmt stattdessen alle gleichzeitig ins Modell auf, lässt aber gleichzeitig deren Koeffizienten bei eingeschränkter Vorhersagekraft gegen Null schrumpfen, um Overfitting zu vermeiden. Wir haben uns aus zwei Gründen gegen dieses Vorgehen und für einen Kompromiss aus subjektiver und datengetriebener Variablenauswahl entschieden: Zum einen ist auch der Regularisierungsansatz inhärent subjektiv, da das Universum der möglichen Prädiktoren vorgegeben werden muss. Zum zweiten dynamisieren wir die Effekte der Koeffizienten über Zeit (siehe Diskussion im Folgenden). Unsere experimentellen Berechnungen haben gezeigt, dass dieses Vorgehen zusammen mit einem Regularisierungsansatz zu extrem instabilen Koeffizientenschätzungen führt.

5 In bekannten strukturellen Modellen haben sich ähnliche Prädiktoren etabliert. Leininger und Kayser (2017) nehmen ebenfalls den Wahlanteil bei der zurückliegenden Bundestagswahl als Prädiktor. Das Kanzlermodell (Gschwend u. Norpoth 2000, 2001) beinhaltet beispielsweise eine Normalwahlkomponente, die über den mittleren Wahlanteil bei den letzten drei zurückliegenden Wahlen operationalisiert wird. In unserem Sample zeigte eine solche, längerfristige Spezifikation eine geringfügig schlechtere Performanz. 
historischen Zusammenhang zwischen Parteianteilen bei aufeinanderfolgenden Wahlen. Der Prädiktor hilft insbesondere technisch zwischen traditionell kleinen und großen Parteien zu diskriminieren, doch auch innerhalb der Cluster kleiner bzw. großer Parteien besteht ein stark positiver Zusammenhang. Gleichzeitig wird deutlich, dass diese Variable nicht ausreicht, um starke Stimmengewinne bzw. -verluste zu erklären, wie sie beispielsweise die Union 2013, die FDP 2013 und die SPD 2009 erlebt bzw. erlitten haben.

Abbildung 1: Bivariate Zusammenhänge zwischen Prädiktoren im strukturellen Modell und Zweitstimmenanteil der Parteien, 1953 - auf Wahlergebnisse der SPD und Union beschränkt

(a)

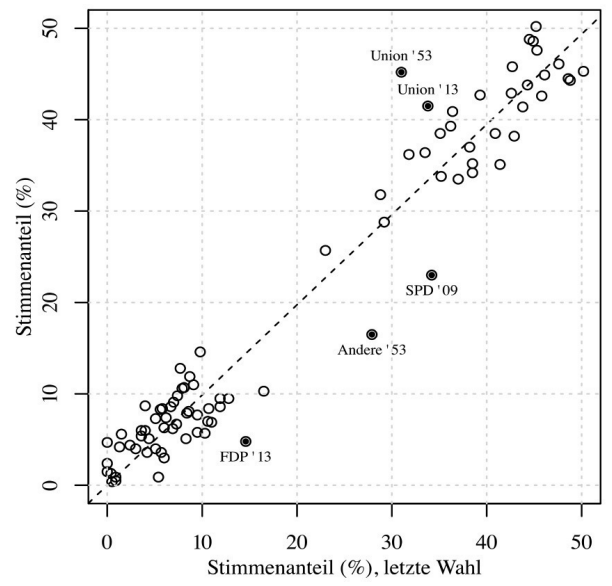

(b)

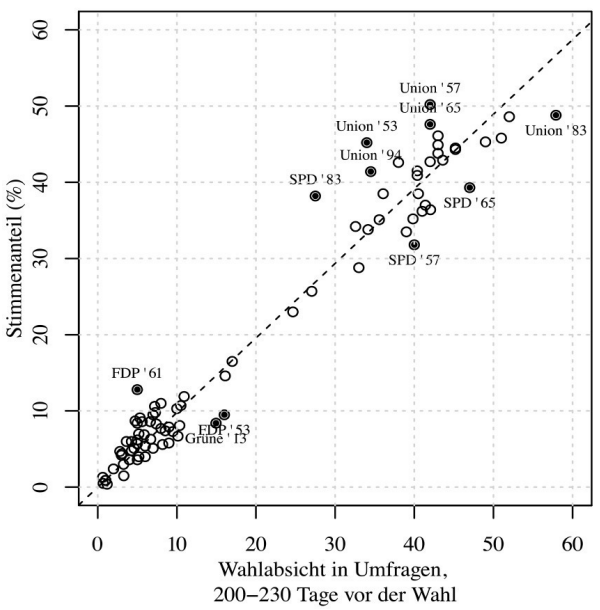

(c)

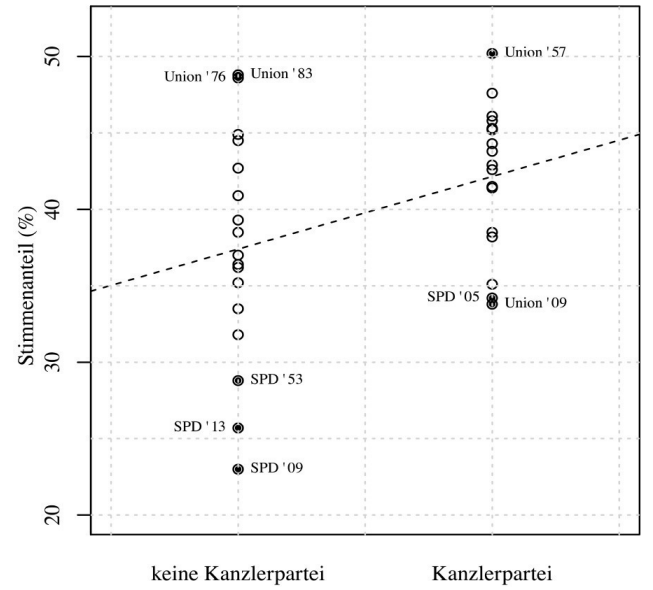

Anmerkungen: Die gestrichelten Linien berichten die vorhergesagten Werte einer linearen Regression. Quelle: eigene Darstellung. 
Der zweite Faktor, die Umfragewerte 230-200 Tage vor der Wahl, bildet in erster Linie kurzfristige Einflussfaktoren ab, die sich auf den Stimmenanteil der Parteien auswirken. Zum einen besteht die jeweilige Wählerschaft einer Partei nicht nur aus Stammwählern, die relativ immun gegenüber bestimmten Themen oder dem speziellen Personal sind, mit denen die Parteien in den Wahlkampf ziehen. Da Parteibindungen üblicherweise stabiler als Wahlentscheidungen sind, müssen selbst Stammwähler immer wieder motiviert werden zur Wahl zu gehen. Zum anderen gibt es auch Wähler, die a priori weniger festgelegt sind, sogenannte Wechselwähler (Schoen 2014), deren jeweilige Wahlentscheidung für eine Partei unter anderem abhängig ist von den speziellen Themensetzungen und dem Spitzenpersonal der Parteien, die diese Themen verkörpern. Zur Operationalisierung dieser kurzfristigen Neigungen verwenden wir den Stand der einzelnen Parteien in der Sonntagsfrage aus veröffentlichten Umfragen (Groß 2010; Schnell u. Novack 2014; Selb u. Munzert 2016). Wir operationalisieren die politische Stimmung unmittelbar vor der heißen Wahlkampfphase als Durchschnitt aller verfügbarer Umfragewerte einer Partei 230-200 Tage vor einer jeden Wahl. ${ }^{6}$ In Panel (b) in Abbildung 1 zeigt sich erneut und wenig überraschend ein starker Zusammenhang zwischen den aggregierten Wahlabsichten 230-200 Tage vor der Wahl und dem tatsächlichen Stimmenanteil bei der darauffolgenden Wahl. Die Varianz der tatsächlichen Anteile um den vorhergesagten Wert ist jedoch, wie dies statistisch auch zu erwarten ist, für die größeren Parteien größer.

Unser dritter Erklärungsfaktor ist institutionell vermittelt. Für die Bewertung der Regierungsarbeit ist auch entscheidend, wer die Regierungskoalition führt (Gschwend u. Norpoth 2000, 2001). Die kanzlerstellende Partei ist also nicht nur eine Partei unter vielen. Kanzlerstellende Parteien bekommen nicht nur Stimmen von Anhängern dieser Partei, sondern darüber hinaus auch Stimmen von Wählern, die diese Partei wählen, um dadurch Einfluss auf die Regierungsbildung und -arbeit auszuüben (Cox 1997). Darüber hinaus wird unterstellt, dass die Kanzlerpartei über diese exponierte Rolle in der Regierung einen Aufmerksamkeitsbonus erhält, der sich ebenfalls in überdurchschnittlicher Unterstützung niederschlagen kann. Dieser besonderen institutionellen Stellung der kanzlerstellenden Parteien tragen wir Rechnung, indem wir einen zusätzlichen Parameter schätzen, der nur den Stimmenanteil dieser Partei beeinflusst. ${ }^{7}$ Panel (c) in Abbildung 1 weist einen deutlich höheren mittleren Stimmenanteil für Kanzlerparteien gegenüber der an-

6 Selb u. Munzert (2016) finden empirisch, dass die Ergebnisse von Sonntagsfragen, die zwischen acht und zehn Monaten vor einer Bundestagswahl gestellt wurden, zu geringeren Vorhersagefehlern führen, als die Ergebnisse von Umfragen, die zeitlich näher an der Wahl liegen. Wir lehnen uns in der Variablenkonstruktion an diese empirische Regularität an, wählen jedoch ein etwas nach vorne verschobenes und kürzeres Zeitfenster. Im Fall der Bundestagswahl 1969 wird dieses Fenster um drei Tage erweitert, da ansonsten keine Umfrage erfassbar gewesen wäre.

7 Implementiert wird dies über eine Dummy-Variable, die den Wert 1 für die amtierende kanzlerstellende Partei annimmt. Direkt vor der Wahl 1983 stellte formal die CDU/CSU mit Helmut Kohl den Kanzler. Allerdings interpretieren wir, ebenso wie in Gschwend und Norpoths Kanzlermodell, diese Wahl als Abrechnung mit der von Helmut Schmidt bis zum Herbst 1982 geführten sozialliberalen Koalition. Folglich kodieren wir für die Wahl 1983, abweichend von unserer Regel, die SPD als kanzlerstellende Partei. 
deren großen Nichtkanzlerpartei auf (die kleineren Parteien wurden aus Gründen der Übersichtlichkeit weggelassen).

Da der betrachtete Zeitraum fast 70 Jahre umfasst, stellt sich die Frage, ob die Annahme eines zeitstabilen datengenerierenden Prozesses gerechtfertigt ist. Das System der kollektiven politischen Repräsentation hat sich über die Jahre verändert (Zittel u. Gschwend 2007). Zum einen befördern Prozesse des sozialen Wandels Veränderungen in der traditionellen Wählerbasis von Parteien. Die einzelnen Parteien können sich nicht mehr auf eine bestimmte Wählerklientel verlassen. Zum anderen nimmt die Gruppe der Parteianhänger immer weiter ab (Arzheimer 2006). Der Veränderungsdruck deutet in die Richtung einer stärkeren Personalisierung von Repräsentation. Folglich sollten für die Erklärung des Erfolges von Parteien bei Bundestagswahlen langfristige Faktoren zunehmend weniger wichtig werden, kurzfristige Faktoren dagegen wichtiger. Um dieser Entwicklung Rechnung zu tragen, erlauben wir den $\beta$-Koeffizienten aus Gleichung (2) in einem bayesianischen dynamisch-linearen Modell gemäß eines random walk-Prozesses über die Wahlen zu variieren:

$$
\beta_{w}^{k} \sim N\left(\mu_{w}^{k}, \sigma_{k}^{2}\right)
$$

wobei

$$
\mu_{w}^{k}=\beta_{w-1}^{k}+\gamma_{\mathrm{drift}}^{k}
$$

Die Effekte der Prädiktoren können also so über Bundestagswahlen variieren, dass der durchschnittliche Einfluss des kten Prädiktors eine Funktion des Einflusses bei der vergangenen Wahl, $\beta_{w-1}^{k}$ und eines linearen Trends (erfasst durch den Drift-Parameter $\gamma_{\text {drift }}^{k}$ ist. ${ }^{8}$

Abbildung 2 berichtet die geschätzten $\beta$-Koeffizienten für die vergangenen 17 Wahlen und die kommende Wahl. Wie erwartet, nimmt die Bedeutung des Stimmenanteils bei der letzten Bundestagswahl ab, das Gewicht der Umfragen, die im Vorfeld der jeweiligen Bundestagskampagne veröffentlicht wurden, dagegen zu. Die Koeffizienten entwickeln sich nahezu spiegelbildlich zueinander. Substanziell beobachtet man also, dass die Ergebnisse der vergangenen Wahl immer weniger Vorhersagekraft für die Ergebnisse der kommenden Wahl haben, die Vorhersagekraft der Umfragen hingegen zugenommen hat. Der Effekt für die Kanzlerpartei schwankt über Zeit zwischen 0 und 15 Prozentpunkten, weist allerdings keinen klaren Trend auf. Für die Bundestagswahl 2017 extrapolieren wir den beobachteten Trend: Die Gewichte für die Vorhersage ergeben sich aus den geschätzten Koeffizienten für 2013 und den variablenspezifischen Drift-Parametern, weshalb ein weiter sinkendes Gewicht des Anteils der vergangenen Wahl und ein stärkeres Gewicht der Umfragen zum Einsatz kommen.

8 Eine weniger technisch anspruchsvolle Umsetzung eines Trends über Wahlen hinweg wäre es, die entsprechenden Faktoren mit einer Wahl-Trend-Variable zu interagieren. Das würde allerdings eine starke Annahme über die funktionale Form dieses Veränderungsprozesses implizieren. 
Munzert et al. | Zweitstimme.org. Ein strukturell-dynamisches Vorhersagemodell

Abbildung 2: Koeffizientenschätzungen des strukturellen Modells, 1953-2017

Stimmenanteil bei der letzten Bundestagswahl

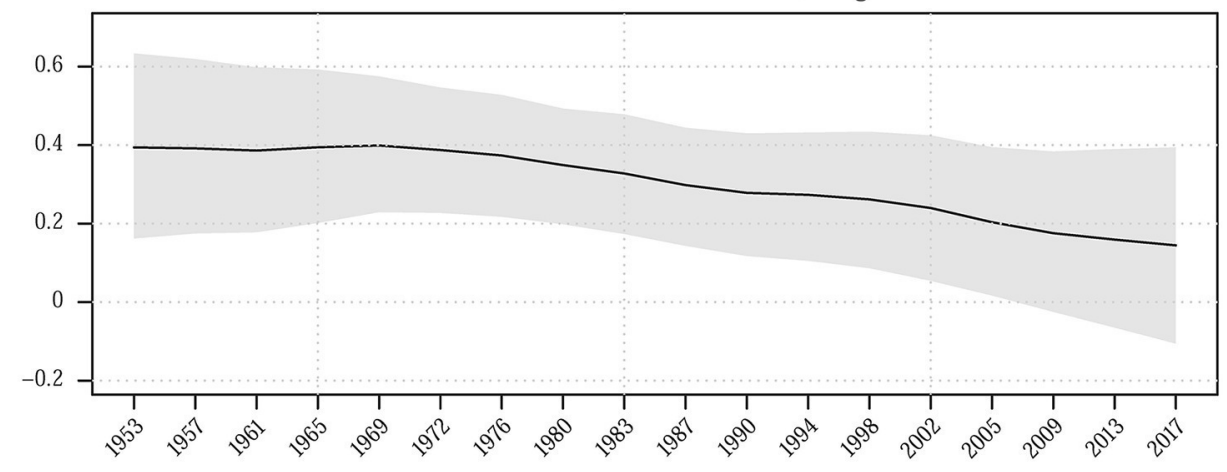

Wahlabsicht in Umfragen, 200-230 Tage vor der Wahl

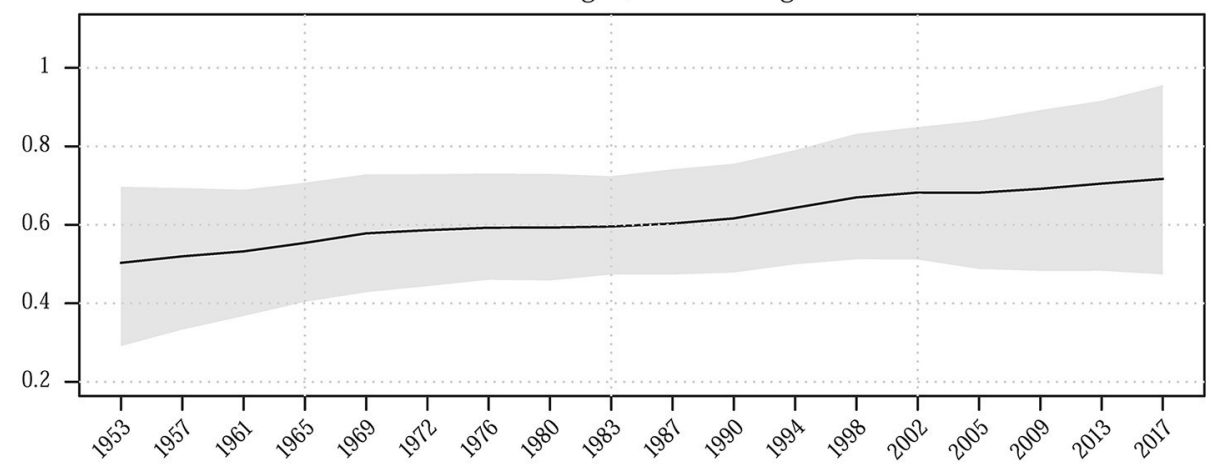

Kanzlerpartei

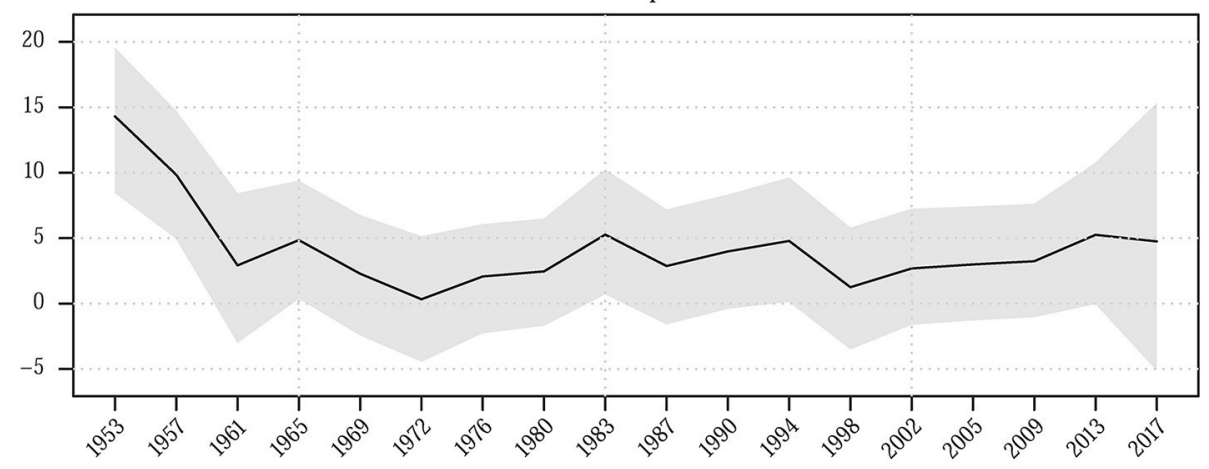

Anmerkungen: Koeffizientenschätzungen des strukturellen Modells, 1953-2017 (vgl. Gleichung [3]). Quelle: eigene Darstellung.

Der Versuch, die Anteile aller Parteien gemeinsam über eine Schätzgleichung vorherzusagen bzw. einen einheitlichen datengenerierenden Prozess über alle relevanten Parteien und Wahljahre zu beschreiben, mag zunächst kontraintuitiv erscheinen. Bei vergangenen Wahlen eingesetzte strukturelle Modelle beschränk- 
ten sich entweder auf die Vorhersage des kombinierten Stimmenanteils der Regierungsparteien (Gschwend u. Norpoth 2000, 2001, 2017; Kayser u. Leininger 2016; Küntzler 2017), oder modellierten die jeweiligen Anteile in separaten Regressionsgleichungen, die dann simultan geschätzt wurden (Jérôme et al. 2013). Tatsächlich birgt unser Vorgehen sowohl Vor- als auch Nachteile. Auf der einen Seite können sinnvollerweise lediglich Prädiktoren verwandt werden, die für alle Parteien und Wahlzeitpunkte definierbar sind, nicht aber solche, die wahl-, aber nicht parteienspezifisch messbar sind. ${ }^{9}$ Außerdem werden im Modell keine mechanischen Vorkehrungen gegen wahlspezifisch unlogische Vorhersagen getroffen (z. B. wenn sich die vorhergesagten Wahlanteile nicht zu exakt $100 \%$ summieren). Aus diesem Grund korrigieren wir die resultierenden Vorhersagen ex post, indem die einzelnen vorhergesagten Wahlanteile anhand deren Summe normalisiert werden.

Aus unserer Sicht überwiegen jedoch die Vorteile unseres Ansatzes: Er ermöglicht Vorhersagen für die Anteile aller relevanten Parteien, die zur Wahl antreten - auch für solche mit eingeschränkter Historie, wie zum Beispiel die AfD im Rahmen der diesjährigen Bundestagswahl. Das wiederum erlaubt, die eigentlichen Fragen von Interesse - siehe Einleitung - präziser zu beantworten. Gleichzeitig erhöht das Modell die Anzahl der Freiheitsgrade, da mehrere Anteile pro Wahl in die Schätzung eingehen. Durch die Dynamisierung der Koeffizientenschätzung können wir schließlich die unrealistische Annahme lockern, dass sich die grundsätzliche Statik der strukturellen Zusammenhänge über die vergangenen Jahrzehnte nicht verändert. Nichtsdestotrotz ist die Vorhersage des strukturellen Modells, wie wir weiter unten zeigen werden, mit erheblicher Unsicherheit behaftet. Im folgenden Abschnitt legen wir dar, wie über den Verlauf der Kampagne veröffentlichte Umfragen effizient ausgewertet werden können, um sowohl die Erwartungen über die jeweiligen Zweitstimmenanteile zu aktualisieren als auch - in Maßen - präzisere Vorhersagen zu generieren.

\subsection{Das Umfrage-Modell}

Die Vorhersagen des strukturellen Modells werden in einem nächsten Schritt durch ein umfragebasiertes Modell dynamisiert. Dazu erweitern wir das von Linzer diskutierte bayesianisch rückwärtsgerichtete random walk-Modell (Linzer 2013). Linzers Modell ist ein Hybrid aus „pooling the polls“-Modellen (Jackman 2005; Pickup u. Johnston 2007; Walther 2015) und strukturellen Vorhersagen. Die Vorhersagen des strukturellen Modells werden dabei als prior-Information für den Wahltag festgesetzt. Ein rückwärtsgerichteter random walk glättet dann, vom Wahltag ausgehend, die Informationen, die aus neu veröffentlichten Umfragen gewonnen werden. Dieses Vorgehen führt zu einer „Mischung“ beider Vor-

9 Geht man beispielsweise davon aus, dass Wirtschaftswachstum Wahlverhalten entscheidend beeinflusst, müsste ein entsprechender Indikator je nach Erwartung mit parteispezifischen Indikatoren (z. B. Regierungspartei) interagiert werden, da es sich bei der Modellierung aller Wahlanteile um ein Nullsummenspiel handelt: Parteiinvariante Indikatoren müssen für einige Parteien positive, für andere aber negative Effekte ausweisen können. 
hersagequellen. Liegt der Wahlzeitpunkt noch weit in der Zukunft, ist die allein auf Umfragen basierende Vorhersage sehr unsicher, da diese über Zeit sehr volatil sind. Dementsprechend wird zunächst der Vorhersage des strukturellen Modells mehr Gewicht beigemessen. Rückt der Wahltag näher, steigt das Vertrauen des Modells in die umfragebasierte Vorhersage, da diese präziser wird. Dieses Vorgehen trägt der Beobachtung Rechnung, dass Sonntagsfragen, die mehrere Monate vor der Wahl durchgeführt werden, keine gute prädiktive Performanz aufweisen (Jennings u. Wlezien 2016). ${ }^{10}$

Unser Modell stellt die erste Anwendung dieses rückwärtsgerichteten random walk-Modells in einem Mehrparteienkontext dar. Dazu müssen einige Anpassungen vorgenommen werden, die wir im Folgenden beschreiben. Die Zeitreihe der Wahl ist definiert durch $T$ Tage zwischen der letzten und der neuen Wahl. $t \in(1$, $\ldots, T)$ ist dabei die Notation für jeden einzelnen Tag, $T$ der Wahltag. Umfrage $y_{t c}$ des Instituts $c \in(1, \ldots, C)$ zum Zeitpunkt $t$ ist ein $(1 \times P)$ Vektor, der die Anzahl der Wahlzusagen für jede der modellierten Parteien $p \in(1, \ldots, P)$ abzählt. Jede Umfrage hat eine Stichprobengröße $N_{c t}$. Die beobachteten Umfrageergebnisse für jede Partei werden dann als Funktion eines latenten Unterstützungsvektors $\alpha_{t}^{*}=\left[\alpha_{t 1}^{*}, \ldots, \alpha_{t P}^{*}\right]$ und eines institutsspezifischen Verzerrungsvektors $\delta_{c}^{*}=\left[\delta_{c 1}^{*}, \ldots, \delta_{C P}^{*}\right]$ modelliert. Der Verzerrungsvektor berücksichtigt, dass Institute bei der ausgewiesenen Unterstützung für bestimmte Parteien systematisch danebenliegen können (Jackman 2005). ${ }^{11}$ Die Umfragewerte folgen daher einer Multinomialverteilung mit Erwartungswert $\alpha_{t}^{*}+\delta_{c}^{*}$ :

$$
y_{t c} \sim \operatorname{Multinomial}\left(\alpha_{t}^{*}+\delta_{c}^{*}, N_{c t}\right) .
$$

Die Vorhersage wird durch die Modellierung von $\alpha_{t}$ dynamisiert. Um der Tatsache, dass sich die geschätzten Parteianteile zu Eins aufaddieren müssen (also die Summe der Einträge des Vektors $\alpha_{t}+\delta_{c}$ für die Multinomialverteilung Eins ergeben muss), Genüge zu tragen, nutzen wir eine zur Analyse von kompositionalen Daten gebräuchliche log-ratio-Transformation (Aitchison 1982) der $\alpha_{t}$-Parameter und Einschränkungen über den Verzerrungsvektor. ${ }^{12}$ Dafür wird die letzte Partei als Referenzpartei $\alpha_{t P}$ verwendet und die Werte für die anderen Parteien folgendermaßen transformiert:

$$
\alpha_{t}=\left[\log \left(\frac{\alpha_{t 1}^{*}}{\alpha_{t P}^{*}}\right), \ldots, \log \left(\frac{\alpha_{t(P-1)}^{*}}{\alpha_{t P}^{*}}\right)\right] .
$$

Diese transformierten Werte werden dann in einen rückwärtsgerichteten random walk integriert:

$$
\alpha_{t} \sim N\left(\alpha_{(t+1)}, S\right)
$$

10 Dies spiegelt sich nicht zuletzt auch darin wider, dass der Einfluss der Umfragekomponente in unserem strukturellen Modell durch die beiden anderen Prädiktoren stark diskontiert wird.

11 Auf diese Instituts- oder „House“-Effekte gehen wir im Folgenden nicht weiter ein, da sie die Vorhersagen kaum beeinflussen. In den Online-Materialien weisen wir die entsprechenden Effekte nach Institut, Wahljahr und Partei getrennt auf.

12 Hierfür wird der Verzerrungsvektor sowohl für Parteien als auch Institute um Null zentriert. Diese Restriktion ist notwendig, um die latente Parteiunterstützung zu identifizieren. 
In dieser Modellierung zeigt die Varianz-Kovarianz-Matrix an, wie stark die für einen Tag identifizierten Unterstützungswerte vom darauffolgenden Tag abhängen. In unserer Spezifikation nutzen wir eine unabhängige Varianzstruktur, sodass $S$ nur von Null verschiedene Elemente $\left(\sigma_{k}^{S}\right)$ auf der Diagonalen besitzt, wobei $1 \leq k \leq P-1 .^{13}$

Die Eleganz dieser rückwärtsgerichteten Formulierung von klassischen „роoling-the-polls"-Modellen besteht darin, dass die Vorhersagen als prior-Information für den Wahltag genutzt werden können. Während bayesianische Modelle mit vorwärtsgerichteter random walk-Implementation prior-Information für den Starttag der Wahlkampagne (bzw. der dynamischen Modellschätzung) benötigen, bedarf es in unserer rückwärtsgerichteten Formulierung priors für den Wahltag. Hier kommen die zuvor präsentierten strukturellen Vorhersagen ins Spiel. Sie beinhalten Erwartungen über das Ergebnis auf Basis historischer Wahlen, die ohne die komplette Zeitreihe der Umfragen auskommen. Dazu werden die Verteilungen der strukturellen Vorhersagen als prior für den Wahltag genutzt. Auch in diesem Fall sollte gewährleistet sein, dass die vorhergesagten Parteianteile zwischen Null und Eins liegen sollten. Dafür arbeiten wir mit einer BetaVerteilung und übertragen Erwartungswert und Standardabweichung der Vorhersage in die shape-Parameter $a_{p}$ und $b_{p}$ der Beta-Verteilung: ${ }^{14}$

$$
\alpha_{T p} \sim \operatorname{Beta}\left(a_{p}, b_{p}\right) .
$$

Um diese Vorhersagen auf die log-transformierte Skala des random walk anzupassen, werden sie ebenfalls transformiert. Die aus diesem Modell resultierende Vorhersage ist dann der latente Zuspruch am Wahltag, $\alpha_{T}$.

Bei ersten Evaluationen des Modells anhand vergangener Wahlen (siehe folgender Abschnitt) stellte sich heraus, dass das Umfragemodell zwar die prädiktive Performanz im Vergleich zu den strukturellen Vorhersagen nochmals deutlich verbessert, die geschätzte Unsicherheit der resultierenden Vorhersagen jedoch zu gering ist. Die mit den Vorhersagen einhergehenden Kredibilitätsintervalle waren also deutlich zu schmal, d. h. beinhalteten den wahren Wert seltener, als dies statistisch zu erwarten wäre. In anderen Worten: Das Modell ist sich „sicherer“, als es auf Basis zurückliegender Vorhersagen sein sollte. Dies liegt daran, dass Umfragen wenige Tage vor der Wahl zwar häufig präzise Vorhersagen liefern, bisweilen jedoch stark danebenliegen. ${ }^{15}$ Der empirische Vorhersagefehler ist also im Mittel deutlich größer, als die Unsicherheit über die geschätzte latente Unterstützung suggerieren würde. Dies spiegelt letztlich den Unterschied zwischen latenter, aber

13 Die Schätzung von Kovarianzen wäre an dieser Stelle prinzipiell auch möglich. Die Vorhersagen werden dadurch allerdings kaum beeinflusst, weshalb wir im Folgenden die sparsamere Spezifikation verwenden.

14 Um sowohl den Erwartungswert für Partei p $\left(\mu_{f p}\right)$ als auch die Standardabweichung $\left(\sigma_{f p}^{2}\right)$ der Vorhersage in der Beta-Verteilung beizubehalten, folgen wir Jackman (2009, S. 55) und nutzen folgende Gleichungen zur Übertragung der Werte: $a_{p}=\left(\frac{1-\mu_{f p}}{\sigma_{f p}^{2}}-\frac{1}{\mu_{f p}}\right) \mu_{f p}^{2}$ und $b_{p}=a_{p}\left(\frac{1}{\mu_{f p}}-1\right)$.

15 So schätzten die letzten Umfragen vor der Wahl 2005 die CDU bei über 40 Prozent ein - das tatsächliche Ergebnis lag dann mit etwa 35 Prozent deutlich darunter. 
eben auf Basis von Surveydaten geschätzter Parteienunterstützung einerseits und tatsächlichem Wahlverhalten andererseits wider. Es gibt viele mögliche Ursachen diese Diskrepanzen (beispielsweise unterschiedliche Surveyteilnahmebereitschaften von Anhängern verschiedener Parteien, kurzfristige Änderungen der Wahlabsichten nach der letzten Umfrage; vgl. dazu auch das „Total-Survey-Error“Framework (Groves et al. 2010; Schnell u. Novack 2014). Um möglichen systematischen Verzerrungen von Umfragen Rechnung zu tragen, nutzen wir deshalb einen ähnlichen Ansatz wie Hanretty et al. (2016) und korrigieren die umfragebasierte Vorhersage um die Fehlervarianz der Vorhersagen vergangener Wahlen. Dies wird praktisch implementiert durch einen zusätzlichen „Sprung“ von der geschätzten latenten Unterstützung am Wahltag zu den finalen Ergebnissen. Die Varianz des Sprungs berechnet sich auf Basis von Umfragen für alle Wahlen seit 1953 (der ersten Wahl mit veröffentlichten Umfragedaten), die in den letzten zwei Tagen vor der Wahl veröffentlicht worden sind.

Nach dieser Korrektur ist unsere Vorhersage der latente Zuspruch am Wahltag plus einem „Sprung“, der die Vorhersageintervalle vergrößert. Um die Beschränkung des latenten Raums zu berücksichtigen, wird die Varianz des Sprungs auf die log-ratio-transformierten latenten Werte $\left(\alpha_{T}\right)$ angewendet und dann rücktransformiert. Die historische Varianz der Fehler der log-ratio-transformierten Werte der „Sonntagsfrage“ seit 1953 beträgt 0,18. Diesen Wert nutzen wir als Varianz des „Sprungs“. Die Vorhersage für jede Partei ist dann $f_{p}=\alpha_{T p}+s_{p}$, mit $s_{p} \sim N(0,0,0.18)$. Durch die Rücktransformation - damit alle vorhergesagten $f_{p}$ sich wieder auf Eins aufsummieren - ergeben sich schließlich unsere Vorhersagen $f_{p}$.

\section{Daten, Schätzung und Evaluation anhand vergangener Wahlen}

Abbildung 3: Out-of-sample-Vorhersagen des strukturellen Modells von Parteianteilen bei Bundestagswahlen, 1953-2013
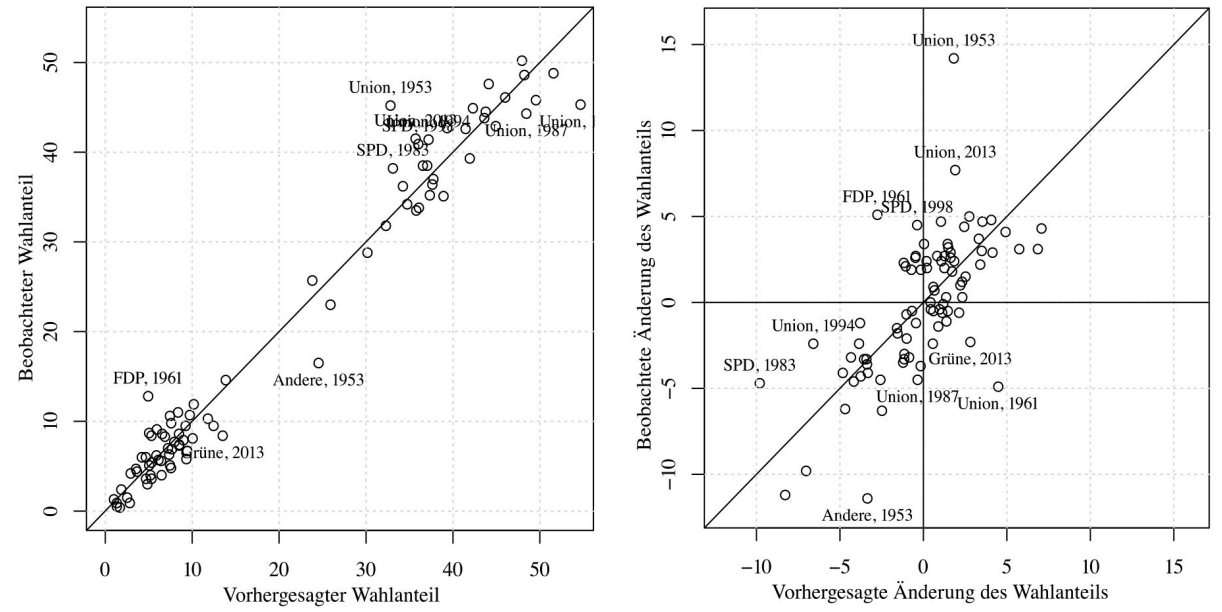

Quelle: eigene Darstellung 
Zur Kalibrierung des strukturellen Modells verwenden wir Daten für alle bisherigen 18 Bundestagswahlen seit 1949. Die Ergebnisse der ersten Bundestagswahl gehen als Indikator für längerfristige Parteienbindung in das Modell ein, werden allerdings nicht selbst geschätzt. Zur Identifikation der Wahlabsicht 230-200 Tage vor der Wahl greifen wir auf Umfragedaten zurück, die von Groß (2010) gesammelt und von Schnell und Noack (2014) erweitert und zur Verfügung gestellt wurden, und füllen Lücken in den Zeitreihen durch weitere Daten auf, die uns vom Institut für Demoskopie Allensbach bereitgestellt wurden. Für Umfragen, die nach 2009 veröffentlicht wurden, verwenden wir Daten der Plattform wahlrecht.de. Umfragen von Instituten, die nur unregelmäßig Ergebnisse veröffentlichen, schließen wir aus und beschränken uns auf Veröffentlichungen vom Institut für Demoskopie Allensbach, von der Forschungsgruppe Wahlen sowie von forsa, Emnid, GMS, Infratest dimap und INSA. Die Umfragen verwenden wir dann auch zur Schätzung des Umfrage-Modells für die Wahlen 2002-2017, und zwar alle, die im letzten Jahr vor der Wahl erhoben wurden.

\section{Abbildung 4: Dynamische Vorhersagen des SPD-Stimmenanteils, Bundestags- wahl 2013}

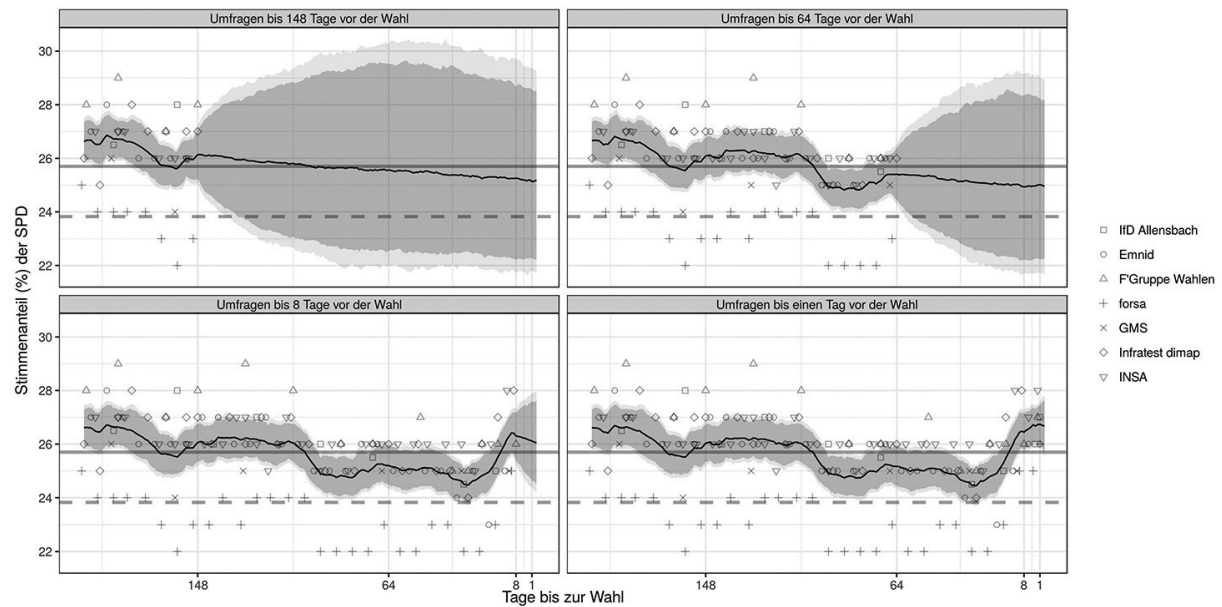

Anmerkungen: Einzelne Symbole markieren Ergebnisse veröffentlichter Umfragen nach Institut. Die durchgezogene Kurve zeigt den Median der latenten Parteiunterstützung aus der posteriori-Verteilung des Modells, die Schattierungen die $90 \%$ - und 95\%-Kredibilitätsintervalle. Das finale Resultat ist mit einer horizontalen durchgezogenen Linie (bei 25,7\%) und die Vorhersage basierend auf dem historischen Modell durch eine gestrichelte Linie (bei 23,8 \%) gekennzeichnet.

Quelle: eigene Darstellung.

Zur Schätzung der Modellparameter und Generierung der vorhergesagten Anteilswerte sowohl im strukturellen als auch im Umfragemodell simulieren wir deren posterior-Verteilung mittels des in JAGS implementierten Markov-Chain- 
Monte-Carlo-Algorithmus (Plummer 2016; R Core Development Team 2013). ${ }^{16}$ Die Schätzung des Umfragemodells basiert auf fünf MCMC-Ketten mit je 200.000 Iterationen nach einer Burn-in-Periode von 150.000 Iterationen, wobei nur jede 100ste Iteration gespeichert wird. Die Konvergenz des verwendeten Gibbs-Samplers überprüfen wir anhand der Gelman-Rubin-Statistik und auf Basis visueller Evidenz (Gelman u. Rubin 1992; Brooks u. Gelman 1998). ${ }^{17}$

Abbildung 3 veranschaulicht den Zusammenhang zwischen den vorhergesagten und realisierten Wahlanteilen von Parteien bei vergangenen Bundestagswahlen. Die Anteile wurden hierfür „out-of-sample“ vorhergesagt, d. h. bei der jeweiligen Schätzung als unbekannt angenommen. Im Mittel über alle Parteien liegt der RMSE (root mean squared error; Wurzel der mittleren quadratischen Abweichung) bei 3,0 Prozent; vereinfacht gesprochen verfehlt also das strukturelle Modell den tatsächlichen Wahlanteil im Durchschnitt um etwa 3 Prozentpunkte. ${ }^{18}$ Im rechten Schaubild von Abbildung 3 wird deutlich, dass einige Beobachtungen vom Modell besonders verfehlt werden. Hier sind nicht die absoluten Wahlanteile, sondern deren Änderungen zwischen aufeinanderfolgenden Wahlen abgetragen. Insbesondere bei der Vorhersage der Anteile der großen Parteien liegt das Modell bisweilen deutlich daneben - im Fall des Erfolgs der Union 2013 (tatsächlich $+7,7 \%$ ) sagte das Modell beispielsweise lediglich einen Zugewinn von 1,9 Prozent voraus. ${ }^{19}$ Ein Blick in die Befragungsdaten für die vom Modell schlecht vorhergesagten Fälle zeigt häufig - wie auch im Falle der Grünen 2013 - einen späten Aufwärts- oder Abwärtstrend, was vom strukturellen Modell nur schlecht aufgefangen werden kann. Um diese Information zu nutzen, werden die strukturellen Vorhersagen mit dem Umfragemodell dynamisiert.

Am Beispiel der Umfragewerte der SPD vor der Bundestagswahl 2013 stellen wir kurz die Funktionsweise der Dynamisierung durch das rückwärtsgerichtete random walk-Modell vor. Abbildung 4 zeigt hierbei die Entwicklung der Modellschätzung über Zeit. Dazu wird das Modell separat für verschiedene Datengrundlagen geschätzt. Zwei zentrale Charakteristiken des Modells sollen anhand dieses Beispiels illustriert werden. Zuerst fällt auf, dass die Unsicherheit über den Zuspruch am Wahltag über Zeit deutlich abnimmt. Während das $95 \%$-Kredibilitätsintervall 148 Tage vor der Wahl von etwas unter 22 Prozent bis etwa 29 Prozent reicht, ist es 8 Tage vor der Wahl deutlich schmaler und umfasst nur noch den Bereich von etwas unter 26 Prozent bis etwa 28 Prozent. Im Fall der SPD 2013 ist das finale Resultat auch in allen Intervallen enthalten. Zweitens zeigt sich, dass anfänglich die strukturellen Vorhersagen noch einen Einfluss auf die Entwicklung der Zustimmung haben, während am Ende der Kampagne das Mo-

16 Daten, Modell-Code und R-Skripte zur Replikation unserer Ergebnisse stellen wir in einem GitHubArchiv frei online unter folgender Adresse zur Verfügung: https://github.com/zweitstimme/btw-2017.

17 Für Modelle, die nicht für die finale Vorhersage verwendet werden, arbeiten wir mit zwei MCMC-Ketten.

18 Der MAE (mean absolute error; mittlere absolute Abweichung), der Ausreißer im Vergleich zum RMSE weniger bestraft, beträgt 2,2 Prozent.

19 Besonders auffällig sind zudem die Ausreißer für die Union und andere Parteien bei der Wahl 1953. Hierbei spielen möglicherweise die Konsolidierung des Parteiensystems und dabei ein besonders starker Stimmengewinn für die Union eine Rolle. 
dell eher dem Umfrage-Trend vertraut. Für die oberen Abbildungen (148 und 64 Tage vor der Wahl) tendiert die Entwicklung der Unterstützung leicht der durch die gestrichelte Linie markierten strukturellen Vorhersagen zu. Im Kontrast dazu ist diese Tendenz zwischen dem achten und letzten Tag vor der Wahl eher gering. Das liegt daran, dass anfänglich die Vorhersage aus dem strukturellen Modell noch relativ viel Information über den finalen Ausgang bereitstellt, während mit zunehmender Präzision der Umfrage dieser mehr Gewicht zugemessen wird. Dieser Effekt ist für kleine Parteien noch stärker, da dort eine präzisere Vorhersage aus dem strukturellen Modell möglich ist. Weitere Beispiele für die Wahlen 20022013 finden sich im Online-Archiv zu diesem Artikel.

Die dynamische Komponente verbessert die Vorhersagekraft unseres Modells für die letzten vier Wahlen erheblich. Tabelle 1 dokumentiert den RMSE für das strukturelle Modell im Vergleich zum Umfragemodell zu unterschiedlichen Zeitpunkten der Kampagne. Der durchschnittliche Fehler des strukturellen Modells für die Wahlen 2002-2013 liegt mit 2,54 relativ niedrig. Das Umfragemodell bietet ähnliche Werte für 148 bis 36 Tage vor der Wahl, verbessert sich dann aber nochmals deutlich in den letzten acht Tagen auf durchschnittlich 1,83. Einen Tag vor der Wahl liegt der RMSE bereits wieder bei 1,98 Prozentpunkten. Mit Ausnahme der Wahl 2005, bei der die Umfragen den Wahlanteil der CDU deutlich überschätzten, zeigt sich für die einzelnen Wahlen dasselbe Muster: Bis 36 Tage vor der Wahl ist die Vorhersagefähigkeit ähnlich gut für das Umfragemodell und das strukturelle Modell, nimmt dann aber für das Umfragemodell erheblich zu. Das ist besonders auffällig für die Wahl 2013, in der das strukturelle Modell stark danebenlag (RMSE 3,47), dieses aber durch die Dynamisierung mittels des Umfragemodells mit einem RMSE von 1,69 und 1,33 einen bzw. acht Tage vor der Wahl aufgefangen werden konnte. Für Vorhersagen ist allerdings nicht nur die erwartete durchschnittliche Abweichung relevant, sondern auch die korrekte Einschätzung der Unsicherheit. Unsere korrigierten $95 \%$-Kredibilitätsintervalle der Vorhersage aus dem Umfragemodell zeigen mit $94 \%$ eine sehr passende Deckung des richtigen Ergebnisses für die letzten vier Wahlen auf - d. h. sie enthalten den wahren Wert nahezu exakt so häufig, wie zu erwarten wäre. ${ }^{20}$

Wie das Umfragemodell die Vorhersagen über Zeit verbessert, stellen wir in Abbildung 5 noch einmal exemplarisch für die Bundestagswahl 2013 dar. Zwei Vorteile des Modells werden dabei besonders deutlich: Zum einen verbessert sich die Vorhersage über Zeit. Das Zentrum der Intervalle liegt für die meisten Parteien und Zeitpunkte zwischen den wahren Werten und den strukturellen Vorhersagen, was eine Reduzierung des Vorhersagefehlers mit näher rückendem Wahltag widerspiegelt. Beispielsweise bewegt sich die Vorhersage für die Grünen bis 36 Tage vor der Wahl etwa auf dem Niveau der strukturellen Vorhersage, nimmt dann jedoch die abstürzenden Umfragewerte auf und nähert sich dem tatsächlichen Ergebnis. Zweitens zeigt sich, dass vor allem für kleine Parteien die Vorhersagen über Zeit deutlich an Präzision gewinnen.

20 Die Deckung für die verschiedenen Abschnitte sind einen Tag vor der Wahl 95 Prozent, acht Tage vor der Wahl 100 Prozent, 36 Tage vor der Wahl 95 Prozent, 64 Tage vor der Wahl 95 Prozent, 92 Tage vor der Wahl 95 Prozent, 120 vor der Wahl 90 Prozent und 148 Tage 90 Prozent. 
Abbildung 5: Entwicklung der Modellvorhersage für die Bundestagswahl 2013, 148 Tage bis einen Tag vor der Wahl
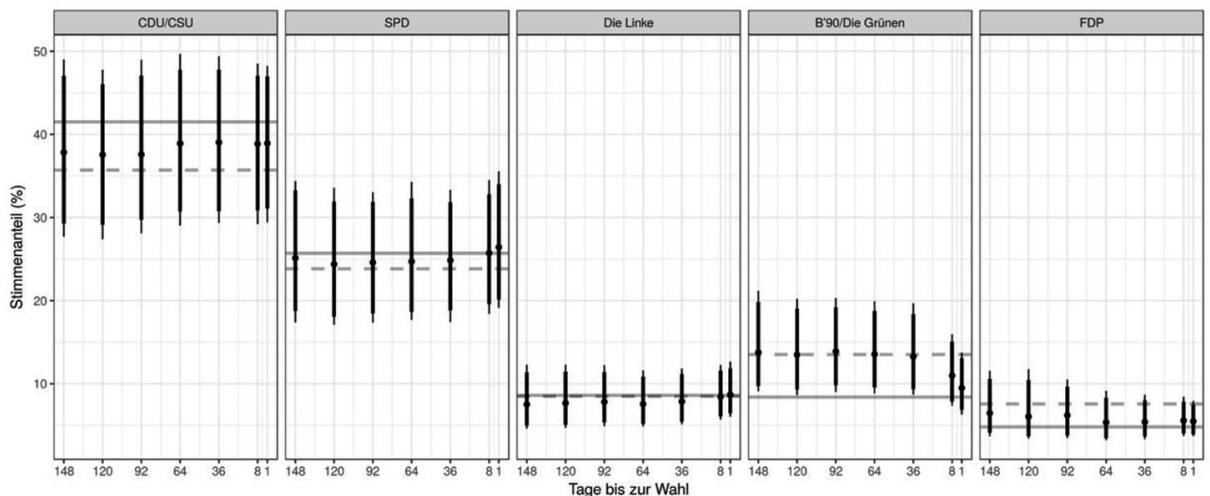

Anmerkungen: Die Punkte geben die Median-Vorhersage an, die dicken bzw. dünnen Balken das $90 \%$ bzw. 95 \%-Kredibilitätsintervall. Das finale Resultat ist mit einer horizontalen durchgezogenen Linie und die Vorhersage basierend auf dem strukturellen Modell durch eine gestrichelte Linie gekennzeichnet. Quelle: eigene Darstellung.

Tabelle 1: Entwicklung des RMSE der out-of-sample-Vorhersagen nach Datengrundlage und Wahl. Strukturelles Modell vs. Umfragemodell, gestaffelt nach Zeitpunkt vor der Wahl

\begin{tabular}{lccccc}
\hline Modell & \multicolumn{5}{c}{ RMSE } \\
\hline & $2002-13$ & 2002 & 2005 & 2009 & 2013 \\
\cline { 2 - 6 } Strukturell & 2,54 & 1,87 & 2,32 & 1,91 & 3,47 \\
Umfrage, 1 Tag vor Wahl & 1,98 & 1,23 & 3,24 & 1,38 & 1,33 \\
Umfrage, 8 Tage vor Wahl & 1,83 & 1,08 & 2,86 & 1,06 & 1,69 \\
Umfrage, 36 Tage vor Wahl & 2,27 & 1,96 & 2,80 & 1,64 & 2,65 \\
Umfrage, 64 Tage vor Wahl & 2,22 & 1,56 & 2,81 & 1,48 & 2,67 \\
Umfrage, 92 Tage vor Wahl & 2,54 & 2,06 & 2,92 & 1,78 & 3,14 \\
Umfrage, 120 Tage vor Wahl & 2,80 & 2,60 & 3,27 & 2,18 & 3,02 \\
Umfrage, 148 Tage vor Wahl & 2,59 & 2,18 & 3,03 & 1,88 & 3,05 \\
\hline
\end{tabular}

Quelle: eigene Darstellung.

Während beispielsweise im Falle der FDP 148 Tage vor der Wahl sowohl ein Wahlerfolg mit über 10 Prozent der Stimmen als auch ein Ausscheiden aus dem Bundestag möglich erscheint, tendiert das Modell 36 Tage vor Wahl zu einem knappen Ergebnis. Interessant ist hierbei, dass der Erwartungswert relativ konstant bleibt und sich nur die Präzision der Vorhersage verändert. Ähnliche Muster erwarten wir auch für die Vorhersagen der Bundestagswahl 2017. 


\section{Vorhersage der Bundestagswahl 2017}

In einem letzten Schritt nutzen wir das Modell, um den Ausgang der Bundestagswahl 2017 vorherzusagen. Dazu verwenden wir an dieser Stelle lediglich Daten, die bis zum 31. Mai 2017, kurz vor der Online-Veröffentlichung dieses Artikels und 116 Tage bzw. knapp vier Monate vor der Wahl zur Verfügung standen. Zur Erzeugung der Wahlabsichtsvariable des strukturellen Modells werden 18 Befragungen verwendet, die zwischen dem 6. Februar 2017 und 8. März 2017 veröffentlicht wurden (und damit in den Zeitraum nach der Verkündung der Kanzlerkandidatur von Martin Schulz fallen). Bei der Vorhersage von Parteianteilen beschränken wir uns auf die Parteien, die laut den Umfragen eine realistische Chance auf den Einzug in den nächsten Bundestag haben (und dabei auch separat ausgewiesen werden): CDU/CSU (ein gemeinsamer vorhergesagter Wahlanteil), SPD, Die Linke, B'90/Die Grünen, FDP und AfD.

\section{Abbildung 6: Dynamische Vorhersagen der Stimmenanteile für die Bundestags- wahl 2017}
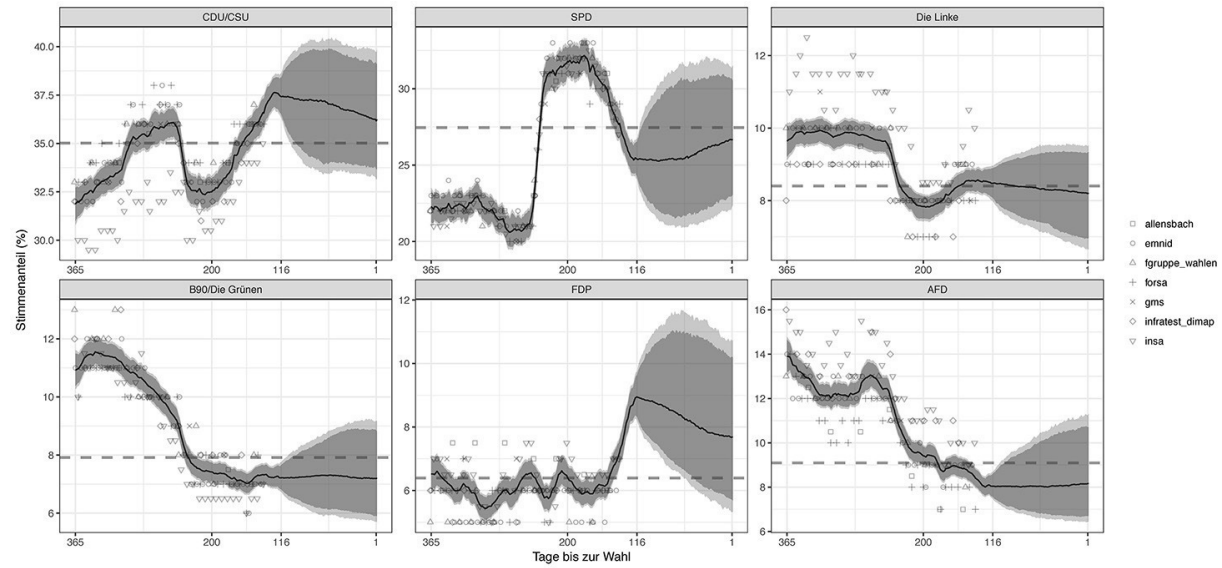

Anmerkungen: Einzelne Symbole markieren Ergebnisse veröffentlichter Umfragen nach Institut. Die durchgezogene Kurve zeigt den Median der latenten Parteiunterstützung aus der posteriori-Verteilung des Modells, die Schattierungen die $90 \%$ - und 95 \%-Kredibilitätsintervalle. Die Vorhersage basierend auf dem strukturellen Modell ist durch eine gestrichelte Linie gekennzeichnet.

Quelle: eigene Darstellung.

Das strukturelle Modell liefert folgende Vorhersage, die als prior-Information für das Umfrage-Modell genutzt wird: CDU/CSU 35,0 Prozent (SD = 4,4), SPD 27,5 Prozent (3,0), Die Linke 8,4 Prozent (2,2), B'90/Die Grünen 7,9 Prozent (2,1), FDP 6,4 Prozent (2,2), AfD 9,1 Prozent (2,3). Abbildung 6 zeigt die Entwicklung der Vorhersage auf Basis der neu verfügbaren Umfragedaten. Um die Dynamik des Umfragemodells besser sichtbar zu machen, deckt die Abbildung den Zeitraum eines ganzen Jahres vor der Wahl ab. Gut sichtbar ist der Schulz-Effekt, ein extremer positiver Schock in der SPD-Zeitreihe, der sich in negativen Schocks der anderen 
Zeitreihen widerspiegelt. Mittlerweile hat sich dieser Effekt jedoch wieder deutlich zurückgebildet - die CDU/CSU liegt in der aktuell geschätzten latenten Zustimmung sogar höher als vor der Ernennung Schulz’ zum Kanzlerkandidaten. Gleichzeitig zeigen die Abbildungen, dass die Vorhersage des strukturellen Modells noch einen substanziellen Einfluss auf die Wahltagsvorhersage ausübt: die Unterstützungskurven schrumpfen noch deutlich in Richtung der strukturellen Vorhersage, sodass wir aktuell ein schwächeres Abschneiden sowohl der CDU/CSU als auch der FDP erwarten würden, als die aktuelle latente Parteienunterstützung erwarten ließe. Grundsätzlich sollte das Gewicht des strukturellen Modells über den Verlauf der Kampagne ab- und das des Umfragemodells weiter zunehmen. Zum Zeitpunkt dieser Vorhersage hält sich der Nutzen der Kombination des strukturellen Modells mit der dynamischen Komponente noch in Grenzen - gemäß den Ergebnissen aus Tabelle 1 würden wir nicht erwarten, dass dieser Schritt zu einer starken Verbesserung der Vorhersagegüte beiträgt. Wir präsentieren hier dennoch die Ergebnisse des kombinierten Modells, um sowohl das Prozedere zu illustrieren als auch die starken Schwankungen in der latenten Parteiunterstützung zu verdeutlichen. Damit verbunden ist auch die große Unsicherheit, die das Modell der Vorhersage zu diesem Zeitpunkt noch beimisst. Die Kredibilitätsintervalle weiten sich zwischen dem Zeitpunkt der Vorhersage und dem Wahltag noch stark aus. Für diesen Zeitraum liegen noch keine Daten vor, dementsprechend groß ist auch die kombinierte Unsicherheit aus dem strukturellen Modell und der Variabilität der Befragungen.

Abbildung 7: Vorhersagen der Stimmenanteile für die Bundestagswahl 2017

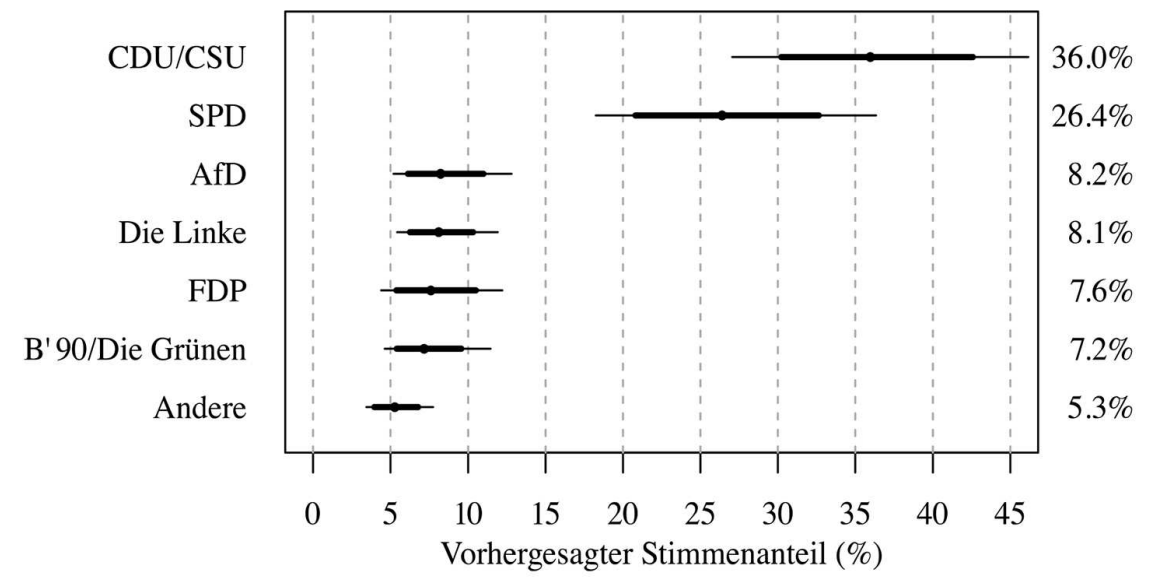

Anmerkungen: $90 \%-(95 \%-)$ Kredibilitätsintervalle durch dicke (dünne) Balken angezeigt. Quelle: eigene Darstellung.

Die aktuellen vorhergesagten Stimmenanteile 158 Tage vor der Wahl sind schließlich nochmals detailliert in Abbildung 7 abgetragen. Demnach käme die CDU/ CSU auf 36,0 Prozent [28,3\%; 44,4 \%], die SPD auf 26,4 Prozent [19,5\%; $34,7 \%]$, die AfD auf 8,2 Prozent [5,6\%; 11,9\%], Die Linke auf 8,1 Prozent 
[5,8 \%; 12,2 \%], die FDP auf 7,6 Prozent [4,9 \%; 11,4 \%], und B'90/Die Grünen auf 7,2 Prozent [5,0\%; 10,5\%] (90\%-Kredibilitätsintervalle in Klammern). ${ }^{21}$ Dabei wird deutlich, dass die mit den aktuellen Vorhersagen verbundene Unsicherheit noch beträchtlich ist. ${ }^{22}$

Tabelle 2: Vorhergesagte Wahrscheinlichkeiten auf Basis der simulierten Stimmenanteile für Ereignisse im Zuge der Bundestagswahl 2017

\begin{tabular}{lc}
\hline Ereignis & Wahrscheinlichkeit (in \%) \\
\hline Größte Partei & 87 \\
CDU/CSU & 13 \\
SPD & \\
Koalitionsmehrheit & 100 \\
CDU/CSU + SPD & 25 \\
CDU/CSU + FDP & 22 \\
CDU/CSU + B'90/Die Grünen & 72 \\
CDU/CSU + B'90/Die Grünen + FDP & 0 \\
SPD + B'90/Die Grünen & 15 \\
SPD + B'90/Die Grünen + Linke & 11 \\
SPD + B'90/Die Grünen + FDP & \\
Scheitern an 5\%-Hürde & 6 \\
FDP & 2 \\
AfD & 6 \\
B'90/Die Grünen & \\
Zugewinn an Stimmenanteilen im Vergleich $z u$ 2013 & 14 \\
CDU/CSU (>41.5\%) & 56 \\
SPD (>25.7\%) & 38 \\
Die Linke (>8,6\%) & 24 \\
B'90/Die Grünen (>8,4\%) & 95 \\
FDP (>4.8\%) & 99 \\
AfD (>4.7\%) & \\
Weitere Ereignisse & 86 \\
Sechs Fraktionen im Bundestag & 33 \\
AfD drittstärkste Fraktion &
\end{tabular}

Quelle: eigene Darstellung.

Ein wichtiger Vorteil der bayesianischen Modellierung und Implementation mittels MCMC-Simulationen ist, dass sich sehr einfach Wahrscheinlichkeiten für alle mit den vorhergesagten Größen direkt verbundene Ereignisse berechnen lassen. Hierzu werden die Ergebnisse der Simulationen herangezogen und aus der relativen Häufigkeit des Eintretens bestimmter Ereignisse deren Wahrscheinlichkeit abgeleitet. Einige dieser Wahrscheinlichkeiten sind in Tabelle 2 aufgelistet. Basierend auf diesen Berechnungen beträgt die Wahrscheinlichkeit für die CDU/CSU, als

21 Die hier berichteten Zahlen beschreiben die Median-, nicht die Mittelwert-Vorhersagen aus den posterior-Verteilungen. Deshalb weicht die Summe der Werte geringfügig von 100 Prozent ab.

22 Die in Abbildung 7 präsentierten Unsicherheitsintervalle sind zudem breiter als die in Abbildung 6 für den Wahltag präsentierten Intervalle. Dies liegt daran, dass letztere die Schock-Anpassung (zur Korrektur möglicher Umfrageverzerrungen) noch nicht berücksichtigt. 
stärkste Fraktion aus der Bundestagswahl hervorzugehen, 87 Prozent, für die SPD lediglich 13 Prozent. Ein Blick auf unterschiedliche Koalitionsoptionen zeigt, dass neben der Großen Koalition aktuell eine Jamaika-Koalition erhebliche Chancen auf eine rechnerische Mehrheit im Parlament hätte, aber auch eine Schwarz-Gelbe Koalition mit 25 Prozent nicht unmöglich scheint. ${ }^{23}$ Gleichzeitig besteht mit Blick auf die kleinen Parteien aktuell für keine ein nennenswertes Risiko, an der 5 \%-Hürde zu scheitern. Gleichzeitig gibt das Modell der FDP, der AfD und der SPD aktuell die höchsten Chancen, im Vergleich zur letzten Wahl Stimmenanteile hinzuzugewinnen und somit als nominelle Gewinner aus der Wahl hervorzugehen. Schließlich sehen 6/7 unserer Simulationen sechs Fraktionen im Bundestag und ein Drittel die AfD als drittstärkste Fraktion.

\section{Vorläufige Diskussion}

Eine abschließende Beurteilung unseres Vorhersagemodells steht zu diesem Zeitpunkt noch aus. Am Abend des 24. September 2017 wird sich herausstellen, ob das Modell - idealerweise besser noch als die unmittelbar vor dem Wahltag veröffentlichten Umfragen - in der Lage war, den Ausgang der Bundestagswahl zu antizipieren. Es ist durchaus möglich, dass das Modell an diesem Test scheitert. Weder verfügen wir über Insiderwissen noch über eine „magische“ Formel, die den Wahlausgang in allen Fällen zuverlässig vorhersagt. Aus wissenschaftlicher Sicht sollte die Präzision einer Vorhersage nicht das alleinige Kriterium zur Beurteilung der Qualität eines Vorhersagemodells darstellen (Lewis-Beck 2005). Die Vorhersage von Wahlergebnissen ist ein Geschäft mit extrem seltenen Ereignissen; es ist somit nicht immer einfach zu beurteilen, ob Erfolg oder Misserfolg eher der Modellspezifikation oder dem Zufall zuzuschreiben sind.

Gleichzeitig sind einige Vorzüge des Modells gegenüber bisherigen Methoden zur Bundestagwahlvorhersage bereits jetzt ersichtlich: Es erlaubt sowohl eine frühzeitige Vorhersage (200 Tage vor der Wahl) durch die historische Komponente als auch eine Aktualisierung der Erwartungen auf Basis neuer Umfragen, kombiniert also die Vorteile struktureller Modelle mit denen von Umfragedaten. Darüber hinaus werden Vorhersagen für alle relevanten antretenden Parteien generiert und nicht nur für den Koalitionsanteil.

Durch das bayesianische Setup ist zudem eine intuitive Ableitung von Wahrscheinlichkeiten jedes beliebigen, auf den prognostizierten Parteianteilen basierenden Ereignisses möglich. Zwar sagt das Modell nichts über den Verlauf und die Erfolgswahrscheinlichkeit von Koalitionsverhandlungen aus (Bräuninger u. Debus 2008, 2012; Debus 2011). Letztlich ist aber die Frage, welche Koalitionen zumindest rechnerisch möglich sein werden, um eine Mehrheit der Sitze im neuen Bundestag zu erhalten, von zentraler Bedeutung für die persönliche Wahlentscheidung und die letztliche Bewertung des Wahlausgangs. Insofern können die Pro-

23 Diese Wahrscheinlichkeiten beziehen sich natürlich lediglich auf die rechnerischen Mehrheiten der Sitze. Dabei wird zwar berücksichtigt, ob ein potenzieller Koalitionspartner die $5 \%$-Hürde überschreitet, jedoch keinerlei strategische Überlegungen zur tatsächlichen Wahrscheinlichkeit der Realisierung einer solchen Koalition durch die beteiligten Parteien angestellt. 
gnosen und daraus abgeleiteten Wahrscheinlichkeiten einen Beitrag dazu leisten, dass Wählerinnen und Wähler bei der Inanspruchnahme eines ihrer wichtigsten Bürgerrechte möglichst gut informiert sind.

Um als hilfreicher Wegweiser für Wählerinnen und Wähler, aber auch für Parteien, Kandidaten und Journalisten zu dienen, ist jedoch auch wichtig, dass sowohl ermittelt als auch kommuniziert wird, mit wie viel Unsicherheit die veröffentlichten Vorhersagen verbunden sind. In den letzten Jahren sind (vor allem umfragebasierte) Prognosemodelle zunehmend in die Kritik geraten, da sie den Ausgang einiger bedeutsamer Wahlen (britische Unterhauswahl 2015; Brexit-Referendum 2016; Trump-Wahl 2016) nicht korrekt vorhersagen konnten. Auf der einen Seite ist nicht davon auszugehen, dass dies mittelfristig das Aus für zuverlässige Wahlvorhersagen bedeutet - im Gegenteil gibt es Hinweise darauf, dass im internationalen Vergleich Umfragedaten nach wie vor ein robuster Prädiktor für Wahlausgänge darstellen (Kennedy et al. 2017). Auf der anderen Seite sind jedoch Vertreter der Wahlvorhersagezunft in der Pflicht, Prognosen mit realistischen Unsicherheiten zu publizieren und diese Unsicherheit auch für die nichtwissenschaftliche Öffentlichkeit verständlich zu machen. Dazu gehört eine Offenlegung der Datengrundlage, Transparenz bei der Modellbildung und Hilfestellung bei der Interpretation der Ergebnisse mit Blick auf die eigentlich interessierenden Größen. Wir versuchen, diesen Kriterien mit unserem Modell gerecht zu werden. Zum einen haben wir technische Vorkehrungen getroffen, um zu zuversichtliche Prognosen zu vermeiden und eine realistischere Quantifizierung von Unsicherheit zu erlangen. Zum anderen stellen wir die Ergebnisse unserer Vorhersage einer breiteren Öffentlichkeit auf dem Internetportal zweitstimme.org zur Verfügung. Das Portal soll auch dazu dienen, Aktualisierungen der Vorhersage im Verlauf der Kampagne zu kommunizieren und interessierten Wahlbeobachtern Werkzeuge an die Hand zu geben, um die mit den Ergebnissen verbundenen geschätzten Wahrscheinlichkeiten für bestimmte Ereignisse intuitiv nachzuvollziehen. Damit hoffen wir im Kontext der Wahlberichterstattung zur kommenden Bundestagswahl einen politikwissenschaftlich fundierten, für die Allgemeinheit nützlichen Beitrag zu leisten.

\section{Literatur}

Aitchison, John. 1982. The Statistical Analysis of Compositional Data. Journal of the Royal Statistical Society Series B 44(2): 139-177. DOI: 10.1007/978-94-009-4109-0.

Arzheimer, Kai. 2006. 'Dead men walking?' Party identification in Germany, 1977-2002. Electoral Studies 25(4): 791-807. DOI: 10.1016/j.electstud.2006.01.004.

Bräuninger, Thomas, und Marc Debus. 2012. Parteienwettbewerb in den deutschen Bundesländern. Wiesbaden: VS Verlag für Sozialwissenschaften.

Bräuninger, Thomas, und Marc Debus. 2008. Der Einfluss von Koalitionsaussagen, programmatischen Standpunkten und der Bundespolitik auf die Regierungsbildung in den deutschen Ländern. Politische Vierteljahresschrift 49(2): 309-38. DOI: 10.1007/ s11615-008-0101-6.

Brooks, Stephen P., und Andrew Gelman. 1998. General Methods for Monitoring Convergence of Iterative Simulations. Journal of Computational and Graphical Statistics 7(4): 434-455. DOI: 10.2307/1390675. 
Campbell, Angus, Philip E. Converse, Warren E. Miller, und Donald E. Stokes. 1960. The American Voter. New York, NY: Wiley.

Converse, Philip E. 1966. The Concept of Normal Vote. In Elections and the Political Order, Hrsg. Angus Campbell, Philip E. Converse, Warren E. Miller, und Donald E. Stokes, 9-39. New York, NY: Wiley.

Cox, Gary W. 1997. Making Votes Count: Strategic Coordination in the World's Electoral Systems. New York, NY: Cambridge University Press.

Debus, Marc. 2011. Parteienwettbewerb, Regierungsbildung und Ergebnisse der Koalitionsverhandlungen nach der Bundestagswahl 2009. In Die Parteien nach der Bundestagswahl 2009, Hrsg. Oskar Niedermayer, 281-306. Wiesbaden: Verlag für Sozialwissenschaften.

Falter, Jürgen W., und Hans Rattinger. 1983. Parteien, Kandidaten und politische Streitfragen bei der Bundestagswahl 1980: Möglichkeiten und Grenzen der Normal-Vote-Analyse. In Wablen und politisches System. Analysen aus Anlass der Bundestagswabl 1980, Hrsg. Max Kaase, und Hans-Dieter Klingemann, 320-421. Opladen: Westdeutscher Verlag.

Gelman, Andrew, und Gary King. 1993. Why Are American Presidential Election Campaign Polls So Variable When Votes Are So Predictable? British Journal of Political Science 23(4): 409-451. DOI: 10.1017/S0007123400006682.

Gelman, Andrew, und Donald B. Rubin. 1992. Inference From Iterative Simulation Using Multiple Sequences. Statistical Science 7: 457-511.

Groß, Jochen. 2010. Die Prognose von Wablergebnissen. Wiesbaden: VS Verlag für Sozialwissenschaften.

Groves, Robert M., Floyed J. Fowler, Jr., Mick P. Couper, James M. Lepkowski, Eleanor Singer, und Roger Tourangeau. 2010. Survey Methodology, 2nd Edition. New York, NY: Wiley.

Gschwend, Thomas, und Helmut Norpoth. 2000. Soll und Haben: Die deutsche Wählerschaft rechnet mit den Parteien ab. In 50 Jahre Empirische Wablforschung in Deutschland. Entwicklung, Befunde, Perspektiven, Daten, Hrsg. Markus Klein, Wolfgang Jagodzinski, Ekkehard Mochmann, und Dieter Ohr, 389-409. Wiesbaden: Westdeutscher Verlag.

Gschwend, Thomas, und Helmut Norpoth. 2001. Wenn am nächsten Sonntag... Ein Prognosemodell für Bundestagswahlen. In Wablen und Wähler: Analysen aus Anlass der Bundestagswahl 1998, Hrsg. Hans-Dieter Klingemann, und Max Kaase, 473-499. Wiesbaden: Westdeutscher Verlag.

Gschwend, Thomas, und Helmut Norpoth. 2005. Prognosemodell auf dem Prüfstand: Die Bundestagswahl 2005. Politische Vierteljahresschrift 46(4): 682-688. DOI: 10.1007/ s11615-005-0308-8.

Gschwend, Thomas, und Helmut Norpoth. 2017. Machtwechsel in Sicht? Die Vorhersage des Kanzlermodells für die Bundestagswahl 2017. Politische Vierteljahresschrift 58(3): 392-406.

Hanretty, Chris, Ben Lauderdale, und Nick Vivyan. 2016. Combining national and constituency polling for forecasting. Electoral Studies 41: 23-43. DOI: 10.1016/j.electstud. 2015.11.019.

Hastie, Trevor, Robert Tibshirani, und Jerome Friedman. 2009. The Elements of Statistical Learning. New York, NY: Springer.

Jackman, Simon. 2005. Pooling the polls over an election campaign. Australian Journal of Political Science 40(4): 499-517. DOI: 10.1080/10361140500302472.

Jackman, Simon. 2009. Bayesian Analysis for the Social Sciences. Chichester: Wiley. 
Jennings, Will, und Christopher Wlezien. 2016. The Timeline of Elections: A Comparative Perspective. American Journal of Political Science 60(1): 219-233. DOI: 10.1111/ ajps.12189.

Jérôme, Bruno, Véronique Jérôme-Speziari, und Michael S. Lewis-Beck. 2013. A PoliticalEconomy Forecast for the 2013 German Elections: Who to Rule with Angela Merkel? Political Science \& Politics 46(3): 479-80. DOI: 10.1017/S1049096513000814.

Kayser, Mark Andreas, und Arndt Leininger. 2016. A Predictive Test of Voters' Economic Benchmarking: The 2013 German Bundestag Election. German Politics 25(1): $106-$ 130. DOI: 10.1080/09644008.2015.1129531.

Kennedy, Ryan, Stefan Wojcik, und David Lazer. 2017. Improving election prediction internationally. Science 355(6324): 515-520. DOI: 10.1126/science.aal2887.

Küntzler, Theresa. 2017. Using Data Combination of Fundamental Variable-Based Forecasts and Poll-Based Forecasts to Predict the 2013 German Election. German Politics. Im Erscheinen. DOI: 10.1080/09644008.2017.1280781.

Lauderdale, Benjamin E., und Drew Linzer. 2015. Under-performing, over-performing, or just performing? The limitations of fundamentals-based presidential election forecasting. International Journal of Forecasting 31(3): 965-979. DOI: 10.1016/j.ijforecast. 2015.03.002.

Leininger, Arndt, und Mark A. Kayser. 2017. Eine länderbasierte Prognose zur Bundestagswahl 2017. Politische Vierteljahresschrift 58(3): 407-417.

Lewis-Beck, Michael, Helmut Norpoth, William Jacoby, und Herbert Weisberg. 2008. The American Voter Revisited. Ann Arbor, MI: University of Michigan Press.

Linzer, Drew A. 2013. Dynamic Bayesian Forecasting of Presidential Elections in the States. Journal of the American Statistical Association 108(501): 124-134. DOI: 10.1080/01621459.2012.737735.

Munzert, Simon 2017. Forecasting elections at the constituency level: A correction-combination procedure. International Journal of Forecasting 33(2): 467-481. DOI: 10.1016/ j.ijforecast.2016.12.001.

Norpoth, Helmut, und Thomas Gschwend. 2003. Against all Odds? The Red-Green Victory. German Politics and Society 21(1): 15-34. DOI: $10.3167 / 104503003782353619$.

Pickup, Mark, und Richard Johnston. 2007. Campaign trial heats as electoral information: Evidence from the 2004 and 2006 Canadian federal elections. Electoral Studies 26(2): 460-476. DOI: 10.1016/j.electstud.2007.03.001.

Plummer, Martyn. 2016. JAGS - Just Another Gibbs Sampler. http://mcmc-jags.sourceforge.net/. Zugegriffen: 24.04.2017.

R Development Core Team (2013). R: A language and environment for statistical computing. R Foundation for Statistical Computing, Vienna, Austria. http://www.r-project.org. Zugegriffen: 24.04.2017.

Schnell, Rainer, und Marcel Noack. 2014. The Accuracy of Pre-Election Polling of German General Elections. Methods, Data, Analyses 8(1): 5-24. DOI: 10.12758/mda.2014.001.

Schoen, Harald. 2014. Wechselwahl. In Handbuch Wablforschung, Hrsg. Jürgen W. Falter, und Harald Schoen, 489-522. Wiesbaden: Springer.

Selb, Peter, und Simon Munzert. 2016. Forecasting the 2013 German Bundestag Election Using Many Polls and Historical Election Results. German Politics 25(1): 73-83. DOI: 10.1080/09644008.2015.1121454. 
Walther, Daniel. 2015. Picking the winner(s): Forecasting elections in multiparty systems. Electoral Studies 40: 1-13. DOI: 10.1016/j.electstud.2015.06.003.

Zittel, Thomas, und Thomas Gschwend. 2007. Der Bundestagswahlkampf von 2005 und Kollektive Repräsentation: Ein kurzer Auftakt zum langen Abschied? In Die Bundestagswabl 2005. Analysen des Wahlkampfes und der Wablergebnisse, Hrsg. Frank Brettschneider, Oskar Niedermayer, und Bernhard Weßels, 119-144. Wiesbaden: VS Verlag für Sozialwissenschaften.

\section{Autorenangaben}

Dr. Simon Munzert

Institut für Sozialwissenschaften

Humboldt Universität zu Berlin

Unter den Linden 6

10099 Berlin

E-Mail: simon.munzert@hu-berlin.de

Dr. Lukas Stötzer

Institut für Politikwissenschaft

Universität Zürich

Affolternstraße 56

8050 Zürich

E-Mail: lukas.stoetzer@uzh.ch

Prof. Thomas Gschwend, Ph.D.

Professor für Politikwissenschaft, quantitative sozialwissenschaftliche Methoden

Universität Mannheim

68131 Mannheim

E-Mail: gschwend@uni-mannheim.de

Marcel Neunhoeffer, M.A.

Fakultät für Sozialwissenschaften

Universität Mannheim

68131 Mannheim

E-Mail: mneunhoe@uni-mannheim.de

Sebastian Sternberg, M.A.

Fakultät für Sozialwissenschaften

Universität Mannheim

68131 Mannheim

E-Mail: ssternbe@uni-mannheim.de 


\title{
Nicht was Du denkst! Was wir wirklich von Wahlvorhersagen lernen können
}

\author{
Oliver Strijbis
}

\begin{abstract}
Not what you think! What we really can learn from election forecasts
Abstract: As this forum demonstrates, the competition between election forecasting models along the US model becomes standard also in Germany. This raises the question about the scientific relevance of this undertaking. It is often argued that election forecasts provide a particularly hard and hence good test for the falsification of theories of electoral behavior. In this contribution, I contest this argument and show that the forecast models presented in this issue hardly contribute to the falsification of theories. Instead I refer to an often overlooked relevance of election forecasts consisting in the models' potential for operationalizing important variables. I illustrate my argument by referring to the literature on prediction markets.
\end{abstract}

Keywords: Bundestag election, forecast, prediction markets, falsification, operationalization Schlagworte: Bundestagswahl, Vorhersage, Prognosemärkte, Falsifizierung, Operationalisierung

\section{Einleitung}

Die Gegenüberstellung von Vorhersagemodellen vor nationalen Wahlen hat in den USA bereits eine lange Tradition (für einen Überblick siehe Campbell u. Lewis-Beck 2008) und wird seit wenigen Wahlen auch in Deutschland zur gängigen Praxis (siehe die Übersicht zu den Bundestagswahlen 2013 in Leininger 2015 und die Beiträge in diesem Heft). Dabei stellt sich die Frage nach dem wissenschaftlichen Sinn dieses Unterfangens (Mayer 2014; Lewis-Beck et al. 2008; Leininger 2015; Campbell 2000). Geht es uns bei den Vorhersagen von Wahlresultaten tatsächlich darum, wissenschaftlichen Fortschritt zu erzielen, wie wir oft versichern, oder werden wir nicht doch eher von sportlichem Ehrgeiz getrieben? ${ }^{1}$ Für letzteres spricht, dass der Vergleich von Wahlvorhersagen in so manchem einem Turnier gleicht. ${ }^{2}$ Dies wird ermöglicht, indem es bei der Evaluation von Wahlvor-

1 Der Autor hat, mit gemischtem Erfolg, Wahl- und Abstimmungsausgänge in Deutschland, der Schweiz und Spanien prognostiziert.

2 Ein Indiz dafür, dass die Prognostik von Wahlvorhersagen einen starken Turniercharakter hat, bietet auch das Geschlechterverhältnis, wenn es um die Autorenschaft von Prognosemodellen geht. Denn Männer sind Wettbewerben gegenüber allgemein deutlich positiver eingestellt (Niederle u. Vesterlund 2011) und bevorzugen eher Spiele mit Turnierformat (Andersen et al. 2012). Bei den in Leininger diskutierten Modellen für die 2013er Wahlen war das Verhältnis von Männern zu Frauen 9:2. Bei den von Campbell aufgelisteten Modellen zu den US Präsidentschaftswahlen im Jahr 2016 lag das Verhältnis bei 15:1. Véronique Jérome wurde dabei doppelt gezählt. Allerdings könnte das Geschlechterverhältnis auch auf die Überrepräsentation von Männern in der quantitativen Politikwissenschaft zurückgeführt werden (z. B. sind zu diesem Zeitpunkt unter den 32 Fellows der quantitativ ausgerichteten Society for Political Methodology nur zwei Frauen zu finden). 
hersagen - für die Sozialwissenschaften ungewöhnlich - recht genaue und unumstrittene Kriterien gibt: Zwar geht es auch darum, ein elegantes und im Idealfall originelles Modell zu präsentieren, doch am Ende gewinnt nur das Modell, das zu einem möglichst frühen Zeitpunkt eine besonders genaue Vorhersage macht. ${ }^{3}$ Geht es uns bei der Prognostik von Wahlen also - Hand aufs Herz - weniger um den wissenschaftlichen Fortschritt sondern in erster Linie um unser Ego oder zumindest um den Spaß am Wettkampf unter Kollegen und Kolleginnen? ${ }^{4}$

Auf diese Frage haben wir üblicherweise verschiedene Antworten bereit. Nicht alle davon sind überzeugend. So verteidigen zum Beispiel mehrere Autoren (Lewis-Beck 2005, S. 145-146; Leininger 2015, S. 677) die Relevanz von Wahlvorhersagen damit, dass diese ihre und des Publikums Neugierde stillen. Weil man aber jede beliebige Forschungsfrage mit Neugierde begründen kann, die Wissenschaft sich jedoch auf besonders bedeutende Fragen fokussieren sollte, fällt diese Begründung schon fast verdächtig schwach aus. Oder wer würde etwa bei einem Projektantrag die Relevanz der Forschungsfrage mit Neugierde begründen? Auch der Verweis darauf, Wahlvorhersagen würden durch politische Akteure nachgefragt und seien damit praktisch relevant (Lewis-Beck 2005, S. 145-146), ist wenig überzeugend. Es ist bekanntlich nicht die Aufgabe der Grundlagenforschung, die Nachfrage auf dem Markt für Politikberatung zu stillen. Dies kann man getrost spezialisierten Unternehmen überlassen.

Viel gewichtiger ist das Argument, dass die Erstellung von Wahlvorhersagen methodische und theoretische Fortschritte in der Wahlforschung, und teilweise darüber hinaus, gebracht hat. Insbesondere heben die Autoren von Wahlvorhersagen die Bedeutung derselben für die Falsifizierung von Theorien hervor (Leininger 2015, S. 678; Kayser u. Leininger 2013; Lewis-Beck u. Stegmaier 2014, S. 284; Sides 2014). In diesem Beitrag werde ich vor allem auf diesen Aspekt der Relevanz von Wahlprognosen fokussieren. Ich werde versuchen zu zeigen, dass die Bedeutung von Wahlvorhersagen für die theoriegeleitete Forschung wesentlich geringer ist, als behauptet. Vorhersagemodelle, so mein Argument, sind nur unter sehr spezifischen Bedingungen für die Falsifizierung von Theorien geeignet. Dabei handelt es sich um Bedingungen, die von den in diesem Forum vorgestellten Modellen in unterschiedlichem Ausmaß und nur teilweise erfüllt werden.

Während dieser Beitrag die Bedeutung von Vorhersagemodellen für die Falsifizierung von Theorien in Frage stellt, identifiziert er eine bisher kaum beachtete Relevanz für die theoriegeleitete Forschung. Ich werde argumentieren, dass Vorhersagemodelle dazu dienen können, Variablen, die in theoretischen Modellen von großer Bedeutung sind, adäquat zu messen. Dieses Argument werde ich anhand empirischer Forschung zu Kampagneneffekten und strategischem Wahlverhalten, welche auf Daten aus Prognosemärkten und strukturellen Modellen abstellt, illustrieren.

3 Für eine Liste von Kriterien siehe Lewis-Beck (2005) und Lewis-Beck und Stegmaier (2014).

4 Gemäß Mayer (2014) macht das Wissen um wissenschaftliche Wahlvorhersagen Politikwissenschaftler zumindest zu interessanteren Gesprächspartnern auf Cocktail-Partys. Wobei Cocktail-Partys ziemlich out sind. 


\section{Die Relevanz von Wahlvorhersagen für die Theorie}

Die Erstellung von Wahlvorhersagen ist für die Politikwissenschaft besonders dann von Bedeutung, wenn sie methodische und theoretische Fortschritte in der Wahlforschung, und teilweise darüber hinaus, generiert. Kaum jemand würde bestreiten, dass man von Vorhersagen etwas lernt. Doch die Frage ist nicht, ob man etwas lernt, sondern ob man nicht mehr lernen würde, wenn man dieselben Ressourcen in ex-post Analysen stecken würde. Zumindest von einigen epistemologischen und methodologischen Fortschritten kann man mit einiger Sicherheit sagen, dass es sie ohne eine Spezialisierung auf Zukunftsvorhersagen wohl nicht gegeben hätte. So wüsste man mit großer Wahrscheinlichkeit viel weniger über sogenannte „house effects“ von Umfrageinstituten (für Deutschland siehe Selb u. Munzert 2016) oder über das Ausmaß von Bestätigungsfehlern (confirmation bias) in der empirischen Politikforschung. Auch besonders die Entwicklung von Methoden, welche für Zukunftsvorhersagen gebildet wurden, aber auch für theoriegeleitete Forschung verwendet werden können (siehe dazu Kapitel 3), wäre bestimmt deutlich weniger weit. So wüsste man wohl kaum um das Problem des „longshot bias“ (Snowberg u. Wolfers 2010) oder systematische Verzerrungen mit automatischen Preismachern (Arnesen u. Strijbis 2015; Dudík et al. 2017), mit denen Prognosemärkte konfrontiert sind. Diese sicherlich unvollständige Liste zeigt, dass die Vorhersageforschung zumindest epistemologisches und methodologisches Wissen generiert.

Wie einleitend erwähnt, wird die Relevanz von Wahlvorhersagen aber vor allem auch damit begründet, dass diese für die Theorie fruchtbar seien. Die theoretische Relevanz von Wahlvorhersagen bestehe vor allem darin, dass Wahlvorhersagen Theorien testen. Der Vorteil von Zukunftsvorhersagen gegenüber ex-post Prognosen besteht darin, dass sie härter und damit aussagekräftiger sind. Denn im Gegensatz zu ex-post Vorhersagen können die Modelle nicht im Nachhinein "gefittet” werden. Auch gibt es bei der Publikation von Zukunftsvorhersagen keine Verzerrung hin zu positiven Resultaten, was der übermäßigen Publikation von „false positives” entgegenwirkt. Damit entfalten ex-ante Publikationen von Vorhersagemodellen durchaus eine willkommene disziplinierende Wirkung.

Doch folgert sich daraus tatsächlich, dass Wahlvorhersagen auch hilfreich sind, um Theorien zu testen? Die Antwort hängt davon ab, wie sehr man Vorhersagemodelle als Operationalisierungen theoretischer Modelle betrachtet. Denn wie alle Modelle sind auch jene zur Vorhersage von Wahlen nur dann für die Falsifizierung nützlich, wenn Theorie und Empirie eng aufeinander abgestimmt sind. Dies geben auch Leininger und Kayser (2017, S. 408) zu bedenken, wenn sie dies für strukturelle Modelle als ausreichend gegeben betrachten: „Denn da strukturelle Modelle [...] theoriebasiert sind und auf historischen Daten beruhen, erfüllen sie die wichtige Funktion, Erwartungen aufzustellen, mit denen die tatsächlichen Resultate schließlich verglichen werden können." Nicht auf Theorien der Wahlforschung basieren Aggregationsmodelle, wie Kombinationen aus Umfragen, Prognosemärkte, Expertenbefragungen oder Wählereinschätzungen. Entsprechend sind sie - im Gegensatz zu strukturellen Modellen - nicht für die Falsifizierung geeignet. Doch selbst unter den sogenannten „strukturellen” Modellen sind viele 
theoretisch nicht besonders wertvoll, weil sie, streng genommen, nicht strukturell sind, sondern einige ihrer Variablen kausal nahe am Wahlentscheid selber liegen (siehe auch Campbell 2000).

Empirische Unterstützung für das Argument, dass Wahlvorhersagen für die Falsifizierung von theoretischen Modellen von Bedeutung sind, gibt es aber von Mayer (2014). So zeigt er unter anderem für die USA die Bedeutung von Wahlvorhersagen für die Bestätigung der Theorie retrospektiven Wählens und das Wissen um „die Natur” des ökonomischen Wählens. ${ }^{5}$ Dies sind bedeutende Erkenntnisse, selbst wenn man bedenkt, dass beide Theoriestränge einander stark überlappen (ein wichtiger Anteil von retrospektivem Wählen ist ökonomisch, und umgekehrt ist ein bedeutender Teil des ökonomischen Wählens retrospektiv). Es ist kein Zufall, dass Wahlvorhersagen, wenn überhaupt, vor allem für die Falsifizierung von den Theorien retrospektiven und ökonomischen Wählens von Bedeutung sind. Denn wenn Vorhersagemodelle am ehesten theoretischen Modellen nachgebildet sind, dann stammen diese aus der Literatur zu retrospektivem und ökonomischem Wählen.

Wie steht es um die drei Modelle, die in diesem Heft vorgestellt werden? Inwiefern erlauben sie eine Falsifizierung von Theorie? Das eleganteste unter den drei Modellen ist das Kanzlermodell von Norpoth und Gschwend (siehe auch Norpoth u. Gschwend 2013; Gschwend u. Norpoth 2005; Norpoth u. Gschwend 2010). Das Kanzlermodell prognostiziert den Stimmenanteil für unterschiedliche Koalitionen auf Basis des langfristigen Wählerrückhalts der jeweiligen Koalition, gemessen als das Mittel der Stimmenanteile in den letzten drei Bundestagswahlen, der Unterstützung für die Kanzlerkandidaten von CDU/CSU und SPD in Umfragen und dem Abnützungseffekt, gemessen anhand der Anzahl Amtsperioden. Das Modell ist elegant, da es nur wenige Variablen beinhaltet. Außerdem hat es einen klaren Fokus auf die Kandidaten und kann daher als ex-ante Test für Theorien, welche Wahlerfolg vor allem mit den Kandidaten erklären, interpretiert werden. Einschränkend aber wirkt der Umstand, dass die Popularität der Kanzlerkandidaten kausal recht nahe an der Wahlentscheidung liegt.

Das Modell von Munzert et al. ist dagegen deutlich weniger elegant. Es beinhaltet sowohl ein strukturelles Modell als auch veröffentlichte Werte der sogenannten „Sonntagsfrage“. Das ermöglicht es zwar, die Stimmenanteile der verschiedenen relevanten Parteien und möglicher Koalitionen vorherzusagen. Indem es aber Umfragedaten verwendet, repräsentiert es kein theoretisches Modell mehr in einem Maße, als dass die Vorhersagen als ex-ante Test interpretiert werden können.

Ähnlich steht es um das in diesem Heft von Kayser und Leininger vorgestellte Modell, das den Wähleranteil der Parteien mit Hilfe von Resultaten aus den Landtagswahlen voraussagt. Als Variablen beinhaltet es den Wähleranteil, welche eine Partei in der vorherigen nationalen Wahl erhalten hat, jenen der letzten Landtags-

5 Außerdem argumentiert Mayer, dass man dank Vorhersagemodellen auf den Abnützungseffekt (Abramowitz 1988) gestoßen ist und etwas über die Bedeutung der Parteienkonvente gelernt hat. Beides sind aber eher empirische Beobachtungen denn theoretische Einsichten, und letztere dürften hauptsächlich für US Präsidentschaftswahlen von Bedeutung sein. Für eine ähnliche Liste siehe auch Lewis-Beck und Stegmaier (2014, S. 286). 
wahl, eine Kanzler-Dummy, das nationale BIP-Wachstum im letzten Quartal, eine Interaktionsvariable der beiden letztgenannten Variablen, die Anzahl der Amtsjahre des Kanzlers bzw. der Kanzlerin sowie eine Interaktionsvariable davon mit einer Partei-Dummy. Das Modell enthält zwar Elemente, die der Theorie des ökonomischen Wählens und jener zum Amtsinhaber-Bonus zugerechnet werden können, indem es aber Wähleranteile von kurz davorliegenden Landtagswahlen aufnimmt, entfernt es sich stark von einem falsifizierbaren theoretischen Modell. Im Gegensatz zu ihrem Benchmark-Modell (Kayser u. Leininger 2013) mögen Kayser und Leininger nun womöglich eine genauere Prognose erstellen, welche zudem nicht nur den Wähleranteil der bisherigen Regierungskoalition, sondern jenen für alle großen und mittleren Parteien vorhersagt. Eine Interpretation mit Bezug auf ein theoretisches Modell scheint nun jedoch nicht mehr möglich.

Die hier vorgestellten Vorhersagemodelle stellen also nur sehr teilweise theoretische Modelle dar. Ihre Relevanz für die Falsifizierung von Theorie ist damit sehr bescheiden. Dieser Punkt scheint mir über die hier besprochenen Modelle verallgemeinerbar zu sein: Das Argument, dass Wahlvorhersagen für die Falsifizierung von Theorie bedeutend sind, ist stark überzogen. Heißt dies, dass Wahlvorhersagen im Allgemeinen der theoriegeleiteten Forschung wenig zu bieten haben? Nicht unbedingt. Denn wie ich im nächsten Kapitel zu zeigen versuchen werde, können Wahlvorhersagen für die Operationalisierung theoretisch bedeutender Variablen verwendet werden.

\section{Verwendung von Wahlvorhersagen zur Operationalisierung von Variablen}

In einem Klassiker der Wahlforschung fragen Gelman und King (1993), weshalb die Umfragen zu US Präsidentschaftswahlen so stark variieren, obwohl ihr Ausgang gut vorhergesagt werden kann. Ihre Antwort darauf ist, dass Wähler und Wählerinnen während eines Wahlkampfes informiert und sich in diesem Prozess über ihre eigentlichen Präferenzen bewusst werden. Und weil dieser Prozess der Aktivierung für unterschiedliche Wählersegmente zu unterschiedlichen Zeitpunkten stattfindet, variieren die Umfragen über die Zeit teilweise stark. Am Ende dieses Aktivierungsprozesses entscheiden sich die Wähler jedoch entlang ihrer Prädispositionen und es resultiert eine „normale Wahl” (Campbell et al. 1960). Indem strukturelle Vorhersagemodelle diese normale Wahl prognostizieren, antizipieren sie ,nur' diesen vorhersehbaren Aktivierungseffekt von Kampagnen (siehe auch Leininger 2015, S. 678). Dasselbe gilt auch, sofern ihre Vorhersagen vor der Kampagne gemacht wurden. Damit können Abweichungen von diesen Vorhersagen als Kampagneneffekte interpretiert werden, die nicht bloß zu einer Aktivierung der Wähler geführt haben, sondern zu einer Wahlentscheidung, die nicht ihren Prädispositionen entspricht. Dieser Effekt wird üblicherweise mit Bezug auf Lazarsfeld et al. (1948) als Überzeugungseffekt (conversion) bezeichnet. Zwar braucht es, streng genommen, keine Wahlvorhersagen, um die normale Wahl zu schätzen, denn strukturelle Modelle können und werden auch ex-post gerechnet. Doch indem Wahlvorhersagen nicht an das Wahlresultat angepasst werden können, ist sichergestellt, dass Überzeugungseffekte der Kampagnen nicht im Modell absorbiert werden. 
Während strukturelle Modelle und frühe Vorhersagen aller Art die normale Wahl vor der Kampagne abschätzen können, ist es möglich mit Prognosemärkten auch während des Wahlkampfes, die bisherigen Überzeugungseffekte eingerechnet, eine normale Wahl zu messen. Entscheidend ist dabei, dass die „Bewegung im [Prognosemarkt][...] die unabhängigen (nicht vorhersehbaren) Auswirkungen der Kampagnen und Wahlveranstaltungen reflektieren" (Shaw u. Roberts 2000, S. 264; eigene Übersetzung). Entwicklungen im Wahlkampf, die erwartet werden können, sowie vergangene unerwartete Ereignisse, führen hingegen nicht zu Veränderungen in den Marktprognosen, weil sie bereits eingepreist sind. Dies entspricht der Grundannahme der ökonomischen Theorie von effizienten Märkten, wonach alle vorhandenen Informationen in die Preisgestaltung einfließt. Weil Umfragen sowohl auf Aktivierungseffekte als auch auf Überzeugungseffekte reagieren, ermöglicht der Vergleich von Prognosemarkt- mit Umfragedaten eine aussagekräftige Analyse von Kampagneneffekten (Shaw u. Roberts 2000; Arnesen 2011, 2015). Indem Wahlvorhersagen es ermöglichen, die normale Wahl zu schätzen, erlauben sie also einen Test von Theorien zu Kampagneneffekten verschiedenster Art.

Wahlvorhersagen können aber nicht nur zur Analyse von Kampagneneffekten verwendet werden. Sie können auch zur Messung von Erwartungen über den Wahlausgang herangezogen werden. Dies trifft zumindest für Prognosemärkte sowie Wählereinschätzungen und Expertenbefragungen zum Ausgang von Wahlen zu. Denn obwohl sich diese in ihren Samples und/oder der Aggregationsmethode unterscheiden, messen sie allesamt Erwartungen über den Wahlausgang. Die Erwartungen über den Wahlausgang sind wiederum eine zentrale Variable für verschiedene ökonomische und psychologische Theorien des Wählens. Das berühmte Paradox des Wählens (Downs 1957) zum Beispiel besagt, dass es irrational ist zu wählen, weil die Wähler erwarten können, dass ihre Stimmen unter üblichen Gegebenheiten keinen Einfluss auf das Wahlergebnis haben werden. Empirische Arbeiten, die das Paradox des Wählens und daraus abgeleitete Theorien testen wollen, sollten daher ein möglichst gutes Maß für die Erwartungen der Wähler (oder anderer wichtiger Akteure wie Parteieliten) verwenden. Zumindest für vergleichende Studien ist dies oftmals schwierig, weshalb die Literatur auf Proxies zurückgegriffen hat, welche jedoch die Erwartungen nicht immer korrekt abbilden. Mit der steigenden Verfügbarkeit von Vorhersagen, die auf der Aggregation von Erwartungen basieren, stehen aber zunehmend direkte Messungen der Erwartungen auch für vergleichende Analysen zur Verfügung. Strijbis et al. (2016) beispielsweise analysieren den Zusammenhang von Erwartungen über den Ausgang von Volksabstimmungen und Stimmbeteiligung für 56 Volksabstimmungen. Ähnliche Analysen könnten auch für Wahlen durchgeführt werden. So könnten zum Beispiel aggregierte Wählereinschätzungen zu Wahlergebnissen mit der Wahlbeteiligung korreliert werden. Vorhersagen aus Expertenpanels könnten außerdem dazu verwendet werden, den Effekt der Erwartungen von Eliten auf die Wahlbeteiligung zu messen und damit von jenem der übrigen Wahlbevölkerung zu unterscheiden.

Erwartungen über den Wahlausgang sind auch von großer Bedeutung für Theorien strategischen Wählens (Cox 1997; Duverger 1966) und für Theorien 
von Mitläufereffekten (McAllister u. Studlar 1991). Bezüglich der Bundesrepublik ist vor allem strategisches Wählen mit Bezug auf potenzielle Regierungskoalitionen von Bedeutung (Gschwend 2007; Meffert et al. 2011; Meffert u. Gschwend 2011). Diese Literatur macht vor allem Gebrauch von Umfragen und Experimenten, beides Quellen für welche Wahlvorhersagen nicht notwendig sind. Allerdings können auch Prognosemarktdaten für die Analyse von strategischem Wählen und Mitläufereffekten verwendet werden, indem man sich ihre Zeitreihenstruktur zu nutze macht und Prognosemarktvorhersagen mit Wahlabsichten gemäß Umfragedaten vergleicht. So wurde versucht, auf Basis eines Prognosemarktes zu den Wähleranteilen der Parteien bei den Bundestagswahlen 2013 Hypothesen zu strategischem Wählen zu testen (Strijbis et al. 2015). Für die Analyse von strategischem Wählen mit Bezug auf Regierungskoalitionen ist es jedoch zielführender, Prognosemarktdaten zu wahrscheinlichen Koalitionen mit Daten zu Wahlabsicht zu vergleichen, was zum ersten Mal für die spanischen Parlamentswahlen letzten Jahres gemacht wurde (Strijbis et al. im Erscheinen). Da Prognosemärkte und Expertenbefragungen keine aufschlussreiche Analyse von Individualdaten erlauben, liegt ihr Potenzial vor allem in der vergleichenden Aggregatsanalyse. Dasselbe gilt auch für kommerzielle Befragungen von Wählereinschätzungen.

\section{Fazit}

Ein oft hervorgebrachtes Argument für die Relevanz von Wahlvorhersagen ist, dass diese, indem sie vor der Wahl gemacht werden, einen besonders harten und daher guten Test für Wahltheorien darstellen. In diesem Beitrag habe ich dieses Argument in Frage gestellt und versucht zu zeigen, dass die meisten Vorhersagemodelle, die in diesem Forum vorgestellten Modelle eingeschlossen, kaum zur Falsifizierung von Theorien beitragen. Stattdessen habe ich auf eine weitgehend übersehene Relevanz von Wahlvorhersagen hingewiesen, welche in ihrem Potenzial zur Operationalisierung wichtiger Variablen besteht. Dieses Argument wurde vor allem mit Literatur zu Prognosemärkten illustriert. Das ist kein Zufall, denn Aggregationsmodelle haben ein ähnliches, wenn nicht sogar größeres Potenzial als strukturelle Modelle, um Daten für die Operationalisierung theoretisch relevanter Variablen zu generieren. Insofern „rehabilitiert” dieser Beitrag die oftmals als weniger interessant betrachteten Aggregationsmodelle gegenüber den strukturellen Modellen.

Dieser Beitrag hat sich ausschließlich mit der theoretischen Nützlichkeit von Wahlvorhersagen befasst. Der Umstand, dass die in diesem Heft vorgestellten Modelle für die Falsifizierung von Theorien nicht geeignet sind, heißt denn auch nicht, dass sie irrelevant sind. Denn wie ich erwähnt habe, gehen von Wahlvorhersagen auch methodische Innovationen aus. Angesichts der Sophistikation der hier vorgestellten Modelle wäre es keine Überraschung, wenn solche Innovationen auch von ihnen ausgingen. Die Wahlmodelle und ihre Präzision in der Vorhersage sind für die Politikwissenschaft als Disziplin auch insofern von Bedeutung, als dass sie einer wenig informierten Öffentlichkeit zeigen, wie fortgeschritten die Disziplin inzwischen tatsächlich ist. 


\section{Literatur}

Abramowitz, Alan I. 1988. An Improved Model for Predicting Presidential Election Outcomes. Political Science \& Politics 21(4): 843-847. DOI: 10.2307/420023.

Andersen, Steffen, Seda Ertac, Uri Gneezy, John A. List und Sandra Maximiano. 2012. Gender, Competitiveness, and Socialization at a Young Age: Evidence From a Matrilineal and a Patriarchal Society. The Review of Economics and Statistics 95(4): 14381443. DOI: 10.1162/REST_a_00312.

Arnesen, Sveinung. 2011. How prediction markets help us understand events' impact on the vote in US presidential elections. Journal of Prediction Markets 5(3): 42-63. DOI: 10.5750/jpm.v5i3.492.

Arnesen, Sveinung. 2015. Aktivierung und Überzeugung auf Prognosemärkten und in Umfragen. In Aktivierung und Überzeugung im Bundestagswahlkampf 2013, Hrsg. Oliver Strijbis, und Kai-Uwe Schnapp, 33-53. Wiesbaden: Springer VS.

Arnesen, Sveinung, und Oliver Strijbis. 2015. Accuracy and Bias in European Prediction Markets. Italian Journal of Applied Statistics 2(25): 123-138.

Campbell, Angus, Phillip E. Converse, Warren E. Miller, und Donald E. Stokes. 1960. The American Voter. New York, NY: Wiley.

Campbell, James E. 2000. The Science of Forecasting Presidential Elections. In Before the Vote: Forecasting American National Elections, Hrsg. James E. Campbell, und James C. Garand, 169-187. London: Sage.

Campbell, James E., und Michael S. Lewis-Beck. 2008. US presidential election forecasting: An introduction. International Journal of Forecasting 24(2): 189-192. DOI: 10.1016/j. ijforecast.2008.02.003.

Cox, Gary W. 1997. Making votes count: strategic coordination in the world's electoral systems. Cambridge: Cambridge University Press.

Downs, Anthony. 1957. An Economic Theory of Democracy. New York: Harper \& Row.

Dudík, Miroslav, Sébastien Lahaie, Ryan Rogers, und Jennifer Wortman Vaughan. 2017. A Decomposition of Forecast Error in Prediction Markets. arXiv:1702.07810 [cs].

Duverger, Maurice. 1966. Political Parties: Their Organization and Activity in the Modern State. Oxford: Taylor \& Francis.

Gelman, Andrew, und Gary King. 1993. Why Are American Presidential Election Campaign Polls so Variable When Votes Are so Predictable? British Journal of Political Science 23(4): 409-451. DOI: 10.1017/S0007123400006682.

Gschwend, Thomas. 2007. Ticket-splitting and strategic voting under mixed electoral rules: Evidence from Germany. European Journal of Political Research 46(1): 1-23. DOI: $10.1111 / \mathrm{j} .1475-6765.2006 .00641 . x$.

Gschwend, Thomas, und Helmut Norpoth. 2005. Prognosemodell auf dem Prüfstand: Die Bundestagswahl 2005. Politische Vierteljahresschrift 46(4): 682-688. DOI: 10.1007/ s11615-005-0308-8.

Kayser, Mark, und Arndt Leininger. 2013. Economic growth trumps political campaigning: CDU/FDP coalition set to win another election. http://www.hertie-school.org/blog/ mark-kayser/. Zugegriffen: 01.06.2017.

Lazarsfeld, Paul Felix, Bernard Berelson, und Hazel Gaudet. 1948. The People's Choice: How the Voter Makes Up His Mind in a Presidential Campaign. New York; NY: Columbia University Press. 
Leininger, Arndt. 2015. Wissenschaftliche Wahlprognosen - Alternative oder Ergänzung zu Umfragen? Zeitschrift für Parlamentsfragen 46(4): 675-691. DOI: 10.5771/03401758-2015-4-675.

Leininger, Arndt, und Mark A. Kayser. 2017. Eine länderbasierte Prognose zur Bundestagswahl 2017. Politische Vierteljahresschrift 58(3): 407-417.

Lewis-Beck, Michael S. 2005. Election Forecasting: Principles and Practice. The British Journal of Politics and International Relations 7(2): 145-164. DOI: 10.1111/ j.1467-856X.2005.00178.x.

Lewis-Beck, Michael S., Helmut Norpoth, William G. Jacoby, und Herbert F. Weisberg. 2008. The American Voter Revisited. Ann Arbor, MI: University of Michigan Press.

Lewis-Beck, Michael S., und Mary Stegmaier. 2014. US Presidential Election Forecasting. Political Science \& Politics 47(2): 284-288. DOI: 10.1017/S104909651400002X.

Mayer, William G. 2014. What, If Anything, Have We Learned from Presidential Election Forecasting? Political Science \&o Politics 47(2): 329-331. DOI: 10.1017/ S1049096514000134.

McAllister, Ian, und Donley T. Studlar. 1991. Bandwagon, Underdog, or Projection? Opinion Polls and Electoral Choice in Britain, 1979-1987. The Journal of Politics 53(3): 720-741. DOI: $10.2307 / 2131577$.

Meffert, Michael F., und Thomas Gschwend. 2011. Polls, coalition signals and strategic voting: An experimental investigation of perceptions and effects. European Journal of Political Research 50(5): 636-667. DOI: 10.1111/j.1475-6765.2010.01986.x.

Meffert, Michael F., Sascha Huber, Thomas Gschwend, und Franz Urban Pappi. 2011. More than wishful thinking: Causes and consequences of voters' electoral expectations about parties and coalitions. Electoral Studies 30(4): 804-815. DOI: 10.1016/j.electstud.2011.08.001.

Niederle, Muriel, und Lise Vesterlund. 2011. Gender and Competition. Annual Review of Economics 3(1): 601-630. DOI: 10.1146/annurev-economics-111809-125122.

Norpoth, Helmut, und Thomas Gschwend. 2010. The chancellor model: Forecasting German elections. International Journal of Forecasting 26(1): 42-53. DOI: 10.1016/ j.ijforecast.2009.02.008.

Norpoth, Helmut, und Thomas Gschwend. 2013. Chancellor Model Picks Merkel in 2013 German Election. Political Science \&o Politics 46(3): 481-482. DOI: 10.1017/ S1049096513000802.

Selb, Peter, und Simon Munzert. 2016. Forecasting the 2013 German Bundestag Election Using Many Polls and Historical Election Results. German Politics 25(1): 73-83. DOI: 10.1080/09644008.2015.1121454.

Shaw, Daron R., und Brian D. Roberts. 2000. Campaign Events, the Media and the Prospect of Victory: The 1992 and 1996 US Presidential Elections. British Journal of Political Science 30(2): 259-289.

Sides, John. 2014. Four Suggestions for Making Election Forecasts Better, and Better Known. Political Science \& Politics 47(2): 339-341. DOI: 10.1017/S104909651400016X.

Snowberg, Erik, und Justin Wolfers. 2010. Explaining the Favorite-Longshot Bias: Is it Risk-Love or Misperceptions?, Working Paper, National Bureau of Economic Research. http://www.nber.org/papers/w15923. Zugegriffen: 20.04.2017. 
Strijbis, Oliver, Sveinung Arnesen, und Laurent Bernhard. 2016. Using prediction market data for measuring the expected closeness in electoral research. Electoral Studies 44: 144-150. DOI: 10.1016/j.electstud.2016.07.006.

Strijbis, Oliver, Rafael Leonisio, und Sveinung Arnesen. im Erscheinen. El estratégico votante español? Análisis del voto de coaliciones con un mercado de predicción. In Las elecciones generales 2015-2016, Hrsg. Francisco José Llera, Montse Baras, und Juan Montabes, Madrid: CIS.

Strijbis, Oliver, Tom Paltins, und Aguibou Bouare. 2015. Haben die Umfragen das Wahlergebnis beeinflusst? Strategisches Wählen und Mitläufereffekte bei der Bundestagswahl 2013. In Aktivierung und Überzeugung im Bundestagswahlkampf 2013, Hrsg. Oliver Strijbis, und Kai-Uwe Schnapp, 177-193. Wiesbaden: Springer VS.

\section{Autorenangaben}

Dr. Oliver Strijbis

Assistant Professor

Department of Social Sciences

Universidad Carlos III de Madrid

Calle Madrid 128

28903 Getafe (Madrid)

Spain

E-Mail: oliverstefan.strijbis@uc3m.es 


\title{
Studierende im Wahl-Werber-Fieber. Die Erstellung von Wahlplakaten zur Bundestagswahl 2017 im „Praxisseminar Wahlkampagne“
}

\author{
Volker Best
}

Students Struck by Election Fever. Designing Campaign Posters for the Bundestag federal election 2017 as a concept for a hands-on seminar

Abstract: The article introduces the teaching and learning concept for a hands-on seminar in the field of election campaigns. The core idea is to move student groups to develop a strategy for a party and creatively implement it by designing election campaign posters. The concept has been put to the test with Master level students in the run-up of the German Bundestag election in 2017. Cognitive as well as affective teaching and learning goals were extensively accomplished. In particular, the students' experience of competency, autonomy and social inclusion led to a high degree of motivation - in students as well as in academic personnel. However, teaching and learning goals concerning working skills were only partly met. Therefore, the article discusses ideas for an optimization of the concept.

Keywords: Political Science teaching, learning by doing, Bundestag federal election 2017, electoral campaign, electoral poster

Schlagworte: Politikwissenschaftliche Lehre, Praxislernen, Bundestagswahl 2017, Wahlkampf, Wahlplakat

\section{Einleitung ${ }^{1}$}

Wahlen stellen in repräsentativen Demokratien den fundamentalen Moment der (Rück-)Bindung der Herrschaft an das Volk als den politischen Souverän dar. Der vorangehende Wahlkampf ist eine „Hochphase politischer Kommunikation“ (Schoen 2014, S. 661), deren Einfluss auf das Wahlergebnis angesichts schrumpfender Parteibindungen, zunehmender Volatilität und immer später getroffener Wahlentscheidungen tendenziell zunimmt (Schoen 2014, S. 704). Parteienwettbewerb, politische Strategie, Politikinhalte, politische Kommunikation, Mediendemokratie, politische Einstellungen, Wählerverhalten, im Vorgriff auch Koalitionen - all dies verbindet sich in Wahlkämpfen und macht diese zu einem relevanten Gegenstand nicht nur für die Forschung, sondern gerade auch für die Lehre politischer Systeme.

Dieser Beitrag stellt das im Wintersemester 2016/17 im Master-Studiengang „Politikwissenschaft“ an der Rheinischen Friedrich-Wilhelms-Universität Bonn realisierte Lehr-Lern-Konzept eines „Praxisseminar Wahlkampagne“ vor und re-

1 Der Autor bedankt sich herzlich bei den zwei anonymen Gutachtern für wertvolle Verbesserungsvorschläge und bei den Seminarteilnehmerinnen und -teilnehmern für die Bereitstellung fehlender Informationen. 
flektiert dieses. Das Seminar war im Wahlpflichtmodul „Prozesse und Politikfelder" verortet und wurde im wöchentlichen Rhythmus durchgeführt. In diesem Seminar erarbeiteten 32 Studierende als Studienleistung in Gruppen von vier bis sechs Personen eine Wahlkampfstrategie jeweils einer Partei für die Bundestagswahl 2017 und setzten diese in Form einer Plakatkampagne gestalterisch um. Der Beginn der Durchführung ein knappes Jahr vor der Bundestagswahl stellte sicher, dass die Erarbeitung der Strategien und Plakate nicht durch die Wirklichkeit überholt wird.

Im Folgenden werden zunächst die Lehr- bzw. Lernziele des Seminars dargestellt (2.) sowie der Seminarablauf, die eingesetzten Sozialformen und Lehr-/Lernmethoden erläutert (3.). Im Anschluss werden die Plakatkampagnen der Projektgruppen für die einzelnen Parteien vorgestellt (4.) und das Feedback der Studierenden zum Seminar präsentiert (5.). Der Beitrag schließt mit einem Fazit zum „Praxisseminar Wahlkampagne“ und einem Ausblick für eine zukünftige Durchführung dieser Veranstaltung (6.).

\section{Lehr- bzw. Lernziele}

Mit Bloom et al. (1973) wird meist zwischen kognitiven, affektiven und psychomotorischen Lehr- bzw. Lernzielen unterschieden. Für letztere wird hier die Bezeichnung arbeitstechnische Lernziele von Johansen et al. (2010, S. 15) verwendet. Die affektiven Lehrziele im „Praxisseminar Wahlkampagne“ bestanden darin:

1. die Lern- und Arbeitsmotivation der Studierenden durch Autonomieerleben, Kompetenzerleben und soziale Eingebundenheit zu verbessern (Deci u. Ryan $1993)^{2}$

2. eine positive Einstellung der Studierenden zu Teamarbeit, kreativem Arbeiten sowie unkonventionellen Lehr-Lern-Situationen zu fördern,

3. bei den Studierenden eine besondere Aufmerksamkeit für den Bundestagswahlkampf $2017 \mathrm{zu}$ wecken.

Was die kognitiven Lehrziele betrifft, so sollten die Studierenden:

1. die Plakatkampagnen zu vergangenen Bundestagswahlen (1998/2002 bis 2013) kennen (als Referenzrahmen und Startpunkt für eigene kreative und gestalterische Überlegungen),

2. die zugrundeliegenden Strategien von Plakatkampagnen zu Bundestagswahlen nachvollziehen (als Referenzrahmen und Startpunkt für eigene strategische Überlegungen),

3. die Wirkmechanismen von Wahlplakaten verstehen,

2 Decis und Ryans Selbstbestimmungstheorie der Motivation postuliert drei grundlegende Bedürfnisse nach Autonomie, Kompetenz und sozialer Eingebundenheit als essenziell für die intrinsische Motivation von Lernenden. Autonomiefördernde Lernumwelten lassen Spielräume bei der Auswahl von Inhalten und/oder der Lernorganisation. Die Erfahrung eigener Kompetenz oder Wirksamkeit wird durch ein angemessenes Anforderungsniveau und Feedback sowie die Realitätsnähe und Problemorientierung von Lernumwelten unterstützt. Soziale Einbindung wird durch eine angenehme Lernatmosphäre begünstigt (Müller 2007, S. 34-36), wie sie nicht zuletzt in Kleingruppen entsteht (Gruber 2007, S. 61). 
4. die Grundzüge des Parteienwettbewerbs mit Blick auf die Bundestagswahl 2017 erfassen,

5. das Programm, das Personal, das Image und die Wählerstruktur der jeweiligen Partei kennen,

6. die Koalitionsoptionen und die dominante Zielorientierung ${ }^{3}$ der Partei in Bezug auf die Bundestagswahl 2017 identifizieren (um diese bei der Strategieentwicklung einbeziehen zu können),

7. diesen Grundlagen Rechnung tragend eine Strategie und mögliche Gewinnerthemen der Partei für die Bundestagswahl 2017 herausarbeiten,

8. diese Strategie und diese Themen kreativ in Wahlplakate umsetzen,

9. die (Zwischen-)Ergebnisse der anderen Gruppen fachkundig diskutieren und begründete Vorschläge zu deren Verbesserung formulieren können,

10. idealiter durch die besondere Aufmerksamkeit für den Bundestagswahlkampf 2017 auch noch ein halbes Jahr nach Seminarende in Form eines Vergleichs der tatsächlichen Kampagnen mit den eigenen bzw. denen der anderen Gruppen dazulernen bzw. das Gelernte Revue passieren lassen und so einen besonders nachhaltigen Lerneffekt erzielen.

Arbeitstechnische Lernziele umfassten:

1. das Aufbereiten von Informationen für andere Studierende in Form von Infopostern und deren Präsentation,

2. die selbstständige Organisation von Gruppenprozessen,

3. den Umgang mit Grafikprogrammen,

4. die Präsentation der eigenen Plakatentwürfe vor der Gruppe, aber auch vor Praktikern der jeweiligen Partei,

5. Feedbackgeben und -nehmen.

\section{Seminarablauf, Sozialformen und Lehr-/Lernmethoden}

Im Zentrum des Seminars stand die Arbeit in den Projektgruppen. Die Projektgruppen wurden weitgehend nach Interesse der Studierenden an den einzelnen Parteien zusammengesetzt. Eine Alternative hätte in einer zufälligen Zuordnung bestanden, was potenziell größere Lerneffekte (im Sinne eines Hineinversetzens in eine weniger naheliegende ideologische Position) ermöglicht hätte. Die Entscheidung für die interessengeleitete Zuordnung erfolgte in der Hoffnung, dass eine stärkere Identifikation mit der über ein gesamtes Semester zu behandelnden Partei eine stärkere Motivation hervorbringen und auf diese Weise den Lerneffekt maximieren würde. Des Weiteren war in den letzten beiden Sitzungen ein Feedback von Parteiakteuren angedacht. Ein darüber hinausgehender potenzieller Nutzen dieses Kontakts für die Studierenden, etwa um ein Praktikum anzubahnen, er-

3 Nach Kaare Strøm (1990) werden hier vote-seeking (Maximieren der Wählerstimmen), officeseeking (Streben nach Regierungsmacht) und policy-seeking (Umsetzung der Parteiprogrammatik in staatliche Politik) unterschieden. Mögliche Zielkonflikte im Wahlkampf können etwa darin bestehen, dass eine klarere inhaltliche Positionierung möglicherweise Wählerstimmen kostet oder eine Negativkampagne unter Umständen zwar Stimmen einbringt, aber eine Möglichkeit zur Regierungsbeteiligung verbaut. 
schien am ehesten bei einer Entscheidung für eine Gruppe aus ideologischer Nähe zu der jeweiligen Partei realisierbar.

Zur Gruppenbildung erwartete die Studierenden in der ersten Sitzung eine Uförmige Sitzordnung, in der mit Schildern Bereiche für die einzelnen Parteien von links nach rechts gemäß ihrer ideologischen Verortung abgeteilt waren. In diesem parlamentsanalogen Setting sollten sich die Studierenden gemäß ihrem Interesse setzen. Zur Wahl standen die fünf in der 18. Legislaturperiode im Bundestag repräsentierten Parteien Die Linke, Bündnis 90/Die Grünen, SPD, CDU und CSU sowie die beiden darüber hinaus im 19. Bundestag mit einiger Wahrscheinlichkeit wieder bzw. erstmals vertretenen Parteien FDP und AfD. Die AfD-Tische blieben zunächst vakant, dann entwickelte sich vorwiegend aus dem zuvor sehr großen rot-grünen Lager heraus aber ein (wohl eher durch akademisches Interesse bzw. unterstellten Spaßfaktor begründeter) Sog Richtung AfD mit dem Resultat, dass diese Gruppe mit sechs Mitgliedern die größte wurde. Für CSU und FDP interessierten sich zunächst nur je zwei Studierende, beide Gruppen konnten aber durch eine (Zwangs-)Zuteilung von Studierenden, die in der ersten Sitzung gefehlt hatten oder noch verspätet um Aufnahme in das Seminar gebeten hatten, auf vier aufgestockt werden, was der minimalen Größe anderer Gruppen entsprach.

In der zweiten Sitzung erstellten die Projektgruppen anhand individuell zugeteilter Vorbereitungstexte eine gemeinsame Situationsanalyse für die jeweilige Partei. In diese Situationsanalyse, die 10-15 Punkte umfassen durfte, sollten die Position der Partei im Parteienwettbewerb mit Blick auf die Bundestagswahl 2017, ${ }^{4}$ die Wählerstruktur der jeweiligen Partei, ihr Programm, ihr Personal, ihr Image, ihre Koalitionsoptionen und ihre gegenwärtige dominante Zielorientierung reflektiert werden. Nach Möglichkeit sollten hieraus auch schon erste strategische Schlussfolgerungen gezogen werden, u. a. in Bezug auf mögliche Gewinnerthemen. Diese wurden in einem für jede Gruppe einzeln eingerichteten Wiki eingestellt. Die Gruppen waren dazu angehalten, arbeitsteilig die Situationsanalysen der (aus ihrer Sicht relevanten) anderen Gruppen zu kommentieren und eventuell in Auseinandersetzung mit deren Ergebnis auftretende Ideen zur Anreicherung der selbst erstellten Situationsanalyse in die eigene Gruppe zu tragen.

Die folgenden drei Sitzungen lieferten Input zu den Themen Wahlkampfstrategien, Wirkungen von Wahlkampfmitteln und Wahlkampagnen 2002-2013. Als Sozialform wurde hier jeweils auf gemischte Gruppen zurückgegriffen. Diese gemischten Gruppen hatten jeweils einen Text zu Hause vorzubereiten und dann im Seminar für die anderen Studierenden in Form eines Infoposters aufzubereiten. Diese Infoposter wurden anschließend in Puzzlegruppen ${ }^{5}$ in fünfminütigen Kurzpräsentationen vorgestellt. Vorteil dieses Vorgehens gegenüber einer Vorstellung im Plenum ist, dass alle Studierende zur Präsentation verpflichtet sind und sich nicht als „Trittbrettfahrer“ ausruhen können. Dementsprechend besteht auch eine

4 Hierzu wurden alle Studierenden über ihre je individuelle spezifischere Lektüre hinaus zum Lesen eines aktuellen Aufsatzes zur Gesamtlage des Parteiensystems aufgefordert.

5 Die „Jigsaw-Methode“ oder auch das „Gruppenpuzzle“ geht auf den US-Sozialpsychologen Elliot Aronson zurück. Dabei werden Inhalte in Gruppen der Zusammensetzung AAA, BBB, CCC usw. aufbereitet und dann von den jeweiligen „Experten“ für A, „Experten“ für B usw. in einer Gruppe der Zusammensetzung ABC präsentiert. http://www.jigsaw.org. Zugegriffen: 20.04.2017. 
hohe Leseverpflichtung im Vorhinein und die Inhalte werden besonders nachhaltig gelernt. Das Format der gemischten Gruppen wurde in dieser Input-Phase den Projektgruppen vorgezogen, weil durch das Verteilen der Projektgruppenmitglieder auf die gemischten Gruppen jedes Mitglied der Projektgruppe einen der behandelten Texte selbst gründlich gelesen und mit anderen durchgesprochen hatte. Somit wurde das Vorhandensein „sektoraler Expertise“ in den Projektgruppen gewährleistet, sodass sich beispielsweise ein Mitglied jeder Projektgruppe mit den Wahlkampagnen 2002, ein anderes mit den Wahlkampagnen 2005 usw. intensiver beschäftigt hatten und mithin entsprechende Erfahrungen in den Gruppenprozess eingebracht werden konnten.

Für die sechste Sitzung wurden wieder Texte auf die Projektgruppenmitglieder verteilt, auf eine Aufbereitung per Infoposter hingegen in Anbetracht des weniger komplexen Inhalts (Praktiker-Tipps zu Wahlkampagnen) sowie aus Zeitgründen verzichtet. Stattdessen wurde den Projektgruppen in dieser Sitzung das Arbeiten an ihrem ersten Plakat-Entwurf ermöglicht. So sollten noch vor der Weihnachtspause und damit bereits in der siebten und achten Sitzung die ersten Entwürfe im Plenum vorgestellt werden, um die dreiwöchige Unterbrechung zur Überarbeitung im Lichte des Peer-Feedbacks und zum Erarbeiten weiterer Ideen nutzbar zu machen. Außerdem sollte vor der Einladung der Parteiakteure - für die wiederum ein gewisser Vorlauf vorzusehen war - sichergestellt werden, dass diesen dann auch etwas Vorzeigbares präsentiert würde. Da sich im Laufe der sechsten Sitzung abzeichnete, dass die meisten Gruppen erst in der achten Sitzung präsentationsfertig sein würden, wurde in Absprache mit den Studierenden die siebte Sitzung für die Präsentation lediglich einer Gruppe und ansonsten für das weitere Arbeiten an den Entwürfen genutzt. Die achte Sitzung, in der statt der geplanten drei bis vier dann sechs Gruppen präsentieren mussten, wurde mit den Teilnehmenden vorab als „open end“ vereinbart, letztlich dauerte sie dann knapp drei Zeitstunden. Nach der Weihnachtspause war nochmals eine Sitzung für die Projektarbeit reserviert. In den folgenden zwei Sitzungen wurden die überarbeiteten und neuen Entwürfe nochmals seminarintern vorgestellt und diskutiert, bevor in den letzten zwei Sitzungen das Feedback aus der Praxis seitens der geladenen Parteiakteure eingeholt wurde.

Im Sinne des „Constructive Alignment“ wurde den Studierenden die Möglichkeit eingeräumt, die Strategie- und Plakatentwicklung zum Gegenstand einer 20bis 25-seitigen Seminararbeit zu machen. Dabei war es den Studierenden freigestellt, inwieweit sie sich die Gruppenergebnisse zu eigen machen oder einzelne Plakate abändern, herauslassen oder hinzufügen wollten.

\section{Plakatkampagnen der Projektgruppen}

In diesem Abschnitt werden die von den Studierenden entworfenen Plakatkampagnen zusammen mit den dahinterliegenden kommunikationsstrategischen Überlegungen vorgestellt, um damit die zentralen inhaltlichen Ergebnisse des Seminares zu beschreiben. Da die Studierenden sich - den Forschungsergebnissen zur Wirkung von Wahlplakaten folgend (Geise u. Brettschneider 2010) - bei der Erstellung der Plakate stark auf Bildplakate fokussierten, sich dabei aber aufgrund des zunächst nur kursinternen Verwendungszwecks nicht durch Urheberrechtsfragen 
unnötig beschränken wollten, können leider nur fünf Plakate bildlich dargestellt werden, eins zu den Grünen, drei zur Linkspartei und eins zur FDP. Für alle anderen Plakate kann lediglich eine verbale Beschreibung erfolgen. Soweit zu rekonstruieren, werden Links zu den verwendeten Bildinhalten angegeben.

Die Anzahl der zu erstellenden Wahlplakate wurde seitens des Dozenten mangels Vorerfahrung mit einer solchen Art von Veranstaltung offengelassen. Im Endeffekt legten die Gruppen zwischen zwei und fünf Plakate vor, die meisten Gruppen erarbeiteten drei Plakate. Inklusive eines weiteren im Rahmen einer Seminararbeit in Reaktion auf das Feedback eines Parteipraktikers ergänzten Plakats wurden insgesamt 29 Plakate realisiert. ${ }^{6}$ Die hierfür verwendeten Programme umfassten Adobe Photoshop, Adobe InDesign, Adobe Illustrator, GIMP (Gnu Image Manipulation Program), PowerPoint und Microsoft Word.

Die SPD-Gruppe konnte bis zum Verzicht des Parteivorsitzenden Sigmar Gabriels auf die Kanzlerkandidatur nicht mit dem Antreten von Martin Schulz als Spitzenkandidat rechnen. Angesichts der schwierigen Ausgangssituation der SPD als Juniorpartner in einer Großen Koalition (Best 2015, S. 95-96), der - jedenfalls bis zum zeitweiligen überraschend starken "Schulz-Effekt“ in den Umfragen über keinerlei anderweitige realistische Machtperspektive verfügte, schienen der Gruppe zwei Punkte zentral: Einerseits galt es die weitreichenden Verhandlungserfolge der SPD in der Großen Koalition herauszustreichen. Andererseits ging es darum zu signalisieren, dass die SPD darüber hinaus inhaltlich auch noch einiges vorhabe, insbesondere in Bezug auf ihren Markenkern soziale Gerechtigkeit. Als erster Slogan entstand „Mehr als den Mindestlohn“, nach einigen anderen Versuchen mit dem Foto eines Bauarbeiters kombiniert. Das Setzen auf eine Personalisierung über Testimonials ${ }^{7}$ statt über den wenig beliebten vermeintlichen Kandidaten Gabriel sollte Volksnähe suggerieren und die Mehrheitsfähigkeit vieler SPD-Vorschläge in der Bevölkerung herausstreichen. Da selbige Strategie bereits im mäßig erfolgreichen Bundestagswahlkampf 2013 verfolgt und die damaligen Plakate als zu gestellt kritisiert worden waren (Lessinger et al. 2015, S. 104-105), bemühte sich die Gruppe um authentischere Bilder. Als ein Kriterium für Authentizität wurde herangezogen, dass der Blick der abgebildeten Personen nicht in die Kamera geht. Da das Corporate Design im Bund nach Meinung der Studierenden zu stark mit der Großen Koalition verbunden wird, verwendeten sie stattdessen jenes des erfolgreichen Landtagswahlkampfs in Rheinland-Pfalz 2016 mit rötlichockerfarbenem Hintergrund. Der erste Teil des Slogans („Mehr als den“) ist weiß gesetzt, der zweite („Mindestlohn“) schwarz (so auch bei den anderen Slogans). Da der „Mehr als...“-Ansatz als wiederkehrendes Kampagnenelement zu einengend erschien, wurde als verbindendes Element zwischen den einzelnen Plakaten stattdessen der dem Parteilogo beigestellte Claim „Dafür wähle ich...“ bzw. „Dafür wählen wir... “ gewählt. Die weiteren Plakate der Serie zeigen ein Großel-

6 Gezählt sind hier nur die jeweils finalen Versionen einer Plakatidee, nicht unterschiedliche Varianten mit z. B. unterschiedlichem Bild. Auch endgültig verworfene Plakatideen wurden hier nicht mitgezählt. Eine Gruppe hatte sich aufgrund strategischer und vor allem gestalterischer Differenzen geteilt, sodass es am Ende insgesamt acht Gruppen gab.

7 Testimonials sind möglichst „glaubhafte Zeitzeugen, die für die Qualitäten eines Kandidaten oder einer Partei bürgen“ (Müller 1997, S. 217). 
ternpaar mit Enkel beim Waldspaziergang ${ }^{8}$ mit dem Slogan „Sichere Renten auch für unsere Enkel“ sowie die Situation eines Arztbesuchs ${ }^{9}$ mit dem Slogan „Gesundheit - von allen für alle“ zur Bewerbung einer paritätischen Bürgerversicherung. Kein genuines SPD-Thema bedient hingegen das Plakat „Sicherheit und Prävention“ mit einem freundlich schauenden Polizisten gehobenen Alters ${ }^{10}$; vielmehr soll hier versucht werden, den Kompetenzvorsprung der Union beim aktuell sehr präsenten Thema „innere Sicherheit“ durch eine eigene Akzentuierung („und Prävention“) so weit wie möglich auszugleichen. Auf die erst Ende Januar 2017, also unmittelbar vor Ende des Wintersemesters, verkündete Spitzenkandidatur von Schulz erwies sich die Gruppe in Form eines Personenplakats ${ }^{11}$ mit dem Slogan „Ein neuer Anfang mit Martin Schulz“ als kurzfristig reaktionsfähig.

Die CDU-Gruppe ging davon aus, dass die 2009 wie 2013 erfolgreiche Strategie der asymmetrischen Demobilisierung und radikalen Personalisierung (Schoofs u. Treibel 2014, S. 65) in Zeiten von Massenzuwanderung und rechtspopulistischen Wahlerfolgen nicht mehr tragfähig sein würde. In Merkels Beliebtheitswerten, die auch nach der Eintrübung durch die Flüchtlingskrise immer noch hoch lagen, sahen die Studierenden nach wie vor ein Pfund der nach zwölf Jahren Regierungsverantwortung programmatisch recht ausgezehrten CDU. Die Kandidatin erschien ihnen mittlerweile aber stärker „begründungsbedürftig“ als 2009 und 2013. Die Gruppe versuchte eine solche Begründung mit einem Plakat unter dem Slogan „Erfahrung ist alles. Unsere Krisenmanagerin.“. Das ganzflächige Bild dazu ist das Gruppenfoto des G7-Gipfels im bayerischen Elmau 2015.12 Die Kanzlerin steht in einem blauen Blazer in der Mitte von acht Staatsmännern im schwarzen Anzug. Der Clou des Motivs: Die Gruppe hat die zwei Herren direkt zur Linken und zur Rechten der Kanzlerin verpixelt und mit weiß gestrichelten Rahmenlinien umgeben. Bei den „Verblichenen" handelt es sich um bereits nicht mehr im Amt befindliche Staats- und Regierungschefs (Barack Obama, François Hollande, David Cameron, Matteo Renzi und Stephen Harper), neben Merkel befinden sich lediglich das außenstehende EU-Führungsduo aus Jean-Claude Juncker und Donald Tusk sowie der japanische Premierminister Shinzō Abe noch im Amt. Die unausgesprochene Botschaft: Wenn überall sonst die „Stützen“ wegbrechen (insbesondere Obama, dessen Nachfolger Donald Trump höchst unkalkulierbar agiert), wird die „mächtigste Frau der Welt“ mehr gebraucht denn je. Dass alle Herren im Bild in die Kamera winken, während Merkel ihre übliche Raute formt, passt optimal. Aufgrund der möglicherweise nicht für alle ohne Erläuterung verständlichen Botschaft könnte dieses Motiv als „Presseplakat“ lanciert werden, mit dem in erster Linie auf Anschlussberichterstattung durch die Medien zu ,smarten' Motiven abgezielt wird. Der zweite Plakatentwurf der Gruppe, der eine Personalisierung Merkels mit der ihrer Partei zugesprochenen Wirtschaftskompetenz verbindet, zeigt die Kanzlerin auf der Cebit in Interaktion mit einem

8 http://bit.ly/spd-rente. Zugegriffen: 20.04.2017.

9 http://bit.ly/spd-arzt. Zugegriffen: 20.04.2017.

10 http://bit.ly/spd-sicher. Zugegriffen: 20.04.2017.

11 http://bit.ly/spd-schulz. Zugegriffen: 20.04.2017.

12 http://bit.ly/cdu-g7. Zugegriffen: 20.04.2017. 
Roboter. ${ }^{13}$ Der Slogan hierzu lautet „Menschlichkeit \& Innovation“, wozu das authentisch wirkende Lächeln Merkels und die menschenähnliche Gestaltung des Robotergesichts passen. Ein drittes, dem Kernthema innere Sicherheit gewidmete Plakat zeigt einen Polizisten und eine Polizistin in der Rückansicht, im Hintergrund ist verschwommen ein älterer Mann zu erkennen. ${ }^{14}$ Der Slogan hierzu lautet „Sicher durch bewegte Zeiten“. Alternativ hätte dieser auch als die Plakate verbindender Claim getaugt, der mehr Substanz geboten hätte als das von der Gruppe zum Transport eines Gemeinschaftsgefühls gewählte, nach Meinung vieler anderer Kursmitglieder letztlich nichtssagende „Mit uns“. Layouterisch hielt sich die CDU-Gruppe an das im Wahlkampf 2013 bewährte Corporate Design der Partei.

Auch die CSU-Gruppe verwendete mit Ausnahme des 2016 neu geordneten Parteilogos das Plakatdesign von 2013 als Vorbild. Als „wunderbar doppelbödig“ empfand die übrige Seminargruppe die Kommunikation des Themas Obergrenze für Flüchtlinge: Eine idyllische Biergartenszene mit drei Personen jüngeren Alters, ein hellhäutiger Mann, eine hellhäutige Frau im Dirndl und einer Frau mit dunklerer Hautfarbe, die sich lachend mit Bierkrügen zuprosten, ${ }^{15}$ wurde mit dem Slogan „GASTFREUNDSCHAFT. Mit Maß.“ unterschrieben. Einzelne Teilnehmende sahen hierin allerdings eher einen gelungenen Bildbeitrag der „heute-show “ als ein potenzielles Wahlkampfplakat. Ein bayerisches Postkartenmotiv wurde auch zum Thema innere Sicherheit herangezogen: ein angeseilter Bergsteiger in Funktionskleidung und Helm am Steilhang vor einem Alpenpanorama, ${ }^{16}$ dazu der Slogan „FREIHEIT braucht SICHERHEIT“. Die schmale Gratwanderung zwischen taktischer Gegenpositionierung zu und Einigkeitsdemonstration mit der Schwesterpartei wurde mittels eines Plakats vollzogen, das in Großaufnahme die Hände Merkels und Seehofers zeigt, während diese nebeneinander vor einem Parteiplakat stehen: ihre zur Raute geformt, seine zur Faust geballt, ${ }^{17}$ dazu der Slogan „GELASSENHEIT braucht STÄRKE“. Ein Personenplakat pries zudem Parteichef und Ministerpräsident Horst Seehofer als „Starke Stimme Bayerns“ an. Hierzu gab es mehrere Entwürfe, die sowohl in den Augen der CSU-Gruppe als auch aus Sicht der übrigen Kursmitglieder alle ihre Stärken und Schwächen hatten hier waren die Studierenden eben auf die von Seehofer im Internet auffindbaren Fotos beschränkt. Als kaum zu bestreitenden vergangenen Erfolg der CSU versuchten die Studierenden, mit der Abschaffung des horizontalen Länderfinanzausgleichs ein sehr abstraktes Thema zu kommunizieren. Mit der Anspielung auf ein bayerisches Volkslied „LÄNDERFINANZAUSGLEICH. Sag beim Abschied leise Servus." wurde hierzu ein passender Slogan gefunden, die Bebilderung mit einem von hinten $\mathrm{zu}$ sehenden, jubelnden Radfahrer in einem sonnenbeschienenen Feld ${ }^{18}$ als Symbol eines hart errungenen Erfolgs blieb aus Sicht einiger anderer Seminarteilnehmender aber zu ambivalent.

13 http://bit.ly/cdu-roboter. Zugegriffen: 20.04.2017.

14 http://bit.ly/cdu-sicher. Zugegriffen: 20.04.2017.

15 http://bit.ly/csu-mass (Bild 3). Zugegriffen: 20.04.2017.

$16 \mathrm{http} / / /$ bit.ly/csu-berg. Zugegriffen: 20.04.2017.

17 http://bit.ly/csu-faust. Zugegriffen: 20.04.2017.

18 http://bit.ly/csu-servus. Zugegriffen: 20.04.2017. 
Die Grünen-Gruppe versuchte sich an „Negative Campaigning“19 gegenüber der CSU. Auf das Gastfreundschafts-Obergrenzen-Plakat der CSU-Gruppe antwortete sie mit einem Piktogramm eines schäumenden Maßkruges und dem Slogan „Das Maß ist voll! Gastfreundschaft kennt keine Grenzen.“. In außerhalb des Seminar-Kontexts besser nachvollziehbarer Weise attackierte die Grünen-Gruppe die Obergrenzen-Forderung in einem weiteren Plakat, das zudem eine Verbindung zur Grünen-Forderung nach einer Beschränkung der steuerlichen Absetzbarkeit von Managergehältern herstellte. Das Piktogramm eines businessmäßig gekleideten Mannes, der wie ein Stabhochspringer rückwärts über eine Stange in Form einer aufsteigenden Diagrammlinie wie in einem Geschäftsbericht springt ${ }^{20}$ wurde mit dem Slogan „Obergrenze?! Nur für Managergehälter.“ versehen. Des Weiteren entstand ein gegen eine Neuauflage der Großen Koalition gerichtetes Plakat, das ein Foto von Schulz und Merkel zeigt, die sich dem Anschein nach in einem Streitgespräch befinden. Ein Graufilter lässt die beiden ohnehin eher unvorteilhaft getroffenen Protagonisten zusätzlich schlecht aussehen und suggeriert eine schlechte Zukunftsperspektive. Übertitelt ist das Foto mit „Wenn zwei sich streiten...“, unter dem Foto folgt der Slogan „Grüne wählen! GroKo stoppen!“. Da explizit nur das Bündnis an sich angegriffen wird, würden sich die Grünen durch ein solches Plakat nach Analyse der Studierenden weder Machtoptionen mit der SPD noch mit der CDU nach der Wahl verbauen. Stilistisch erinnert das Plakat an ebenfalls gegen die Regierungsparteien gerichtete Serien aus den Wahljahren 2009 und 2013, bei denen ein - bei „Negative Campaigning“ durchaus möglicher - Bumerang-Effekt wohl ausblieb (Lessinger u. Holtz-Bacha 2010, S. 108; Dies u. Cornel 2015, S. 113-114). Hauptfokus der Gruppe war allerdings die Mobilisierung der grünen Kernklientel mittels Umweltthemen. Hier wurden der Ausstieg aus der Massentierhaltung und Kohleverstromung als geeignete Themen identifiziert. Während sich mittlerweile alle etablierten Parteien die Energiewende auf die Fahnen schreiben und die Unterschiede etwa hinsichtlich der Vorstellungen zum ErneuerbareEnergien-Gesetz zu technisch sind, um sie allgemeinverständlich, geschweige denn in den wenigen Sekunden Aufmerksamkeit, die einem Wahlplakat im Regelfall zuteilwerden (Lessinger u. Holtz-Bacha 2010, S. 67), zu kommunizieren, erkannten die Studierenden im Ausstieg aus der Kohleenergie eine klar vermittelbare Forderung. Hier könnten die Grünen nach Analyse der Gruppe auch tatsächlich mit einem Alleinstellungsmerkmal aufwarten, da die SPD, die in der ablaufenden Legislaturperiode die Ressorts Umwelt und Energie besetzt, aufgrund von Divergenzen zwischen den beiden zuständigen Ministern keine konsequente Linie vorweisen könne. Der Plakatentwurf zeigt ein in einer Schlammpfütze sitzendes und entsprechend beschmutztes kleines Mädchen. ${ }^{21}$ Der Slogan „Raus aus dem Dreck.“ schließt an die oft humorvolle Plakatgestaltung der Grünen an, der Untertitel „Kohle stoppen - Klima retten. " stellt die Verbindung zu der konkreten Forderung und dem übergeordneten Thema Klimaschutz her. Zwar wurde die Frage aufgeworfen, ob die Grünen tat-

19 „Negative Campaigning“ setzt auf eine Abwertung der politischen Konkurrenz, kann aber auch mit der eigenen Profilierung verbunden werden und gilt in diesem Falle als erfolgsversprechender (Leideker 2010).

20 http://bit.ly/gruene-obergrenze. Zugegriffen: 20.04.2017.

21 http://bit.ly/gruene-dreck. Zugegriffen: 20.04.2017. 
sächlich gut beraten wären, nach der Pädophilie-Debatte im Wahlkampf 2013 mit Kinderbildern zu werben. Die Mehrheit sah darin aber kein Problem und die Gruppe konnte auf gleich zwei Grünen-Plakate aus dem baden-württembergischen Wahlkampf 2016 verweisen, die Kinder zeigten, ohne eine öffentliche Debatte hervorzurufen oder das Ergebnis der Grünen zu schmälern. Für die bildliche Kommunikation zum Thema Massentierhaltung, das beim grünen Mitgliederentscheid zu Themenprioritäten 2013 auf dem ersten Platz landete, dient ein regelrecht in die Kamera hineinschnüffelndes kleines Ferkel mit Dreck an der Schnauze als niedlicher, aber authentischer Sympathieträger (s. Abbildung 1). Der Slogan hierzu lautet „Endlich mal die Sau rauslassen.“, der kleinere Untertitel postuliert „Zeit für eine neue Haltung.“. Die Studierenden kommunizierten so das Anliegen der Grünen auf zwei Bedeutungsebenen: einen Wandel der Haltungsbedingungen und einen Einstellungswandel der Menschen beim Umgang mit Tieren. Der intendierte Effekt bestand darin, dass der Betrachter durch die Vielschichtigkeit gefordert, aber nicht überfordert wird. So soll ein positives Aha-Erlebnis generiert und dadurch die Message nachhaltiger erinnert werden. Das Risiko, dass die grundlegende politische Botschaft durch das kleine „Rätsel“ unverständlich würde, stufte die Gruppe als gering ein. Eine solche „Strategie des zweiten Hinschauens“ oder Nachdenkens, bei der sich die vollständige Botschaft erst bei genauerer Betrachtung offenbart, ist durchaus typisch für die Grünen (HoltzBacha u. Lessinger 2006, S. 115), die ihrer gut ausgebildeten Wählerschaft hier tendenziell etwas mehr abverlangen kann als andere Parteien. Beide Umwelt-Plakate vermeiden durch ihren positiven Ton das 2013 schädliche Image als „Verbots-Partei“. Das Layout aller Plakate orientierte sich an dem 2015 eingeführten Corporate Design der Partei.

Eine besondere Herausforderung stellt die Bundestagswahl 2017 für die FDP dar, die 2013 den Einzug in den Bundestag knapp verpasst hatte. Die FDP reagierte hierauf mit einer umfassenden Analyse der Wahlniederlage, der Erarbeitung eines neuen Leitbildes, das auch in einem komplett neuen Corporate Design Niederschlag fand. Die traditionellen Parteifarben Blau und Gelb wurden durch das Modernität ausstrahlende Magenta ergänzt, der Parteiname wurde von „FDP Die Liberalen“ geändert in „Freie Demokraten - FDP“. Mit diesem „Rebranding “ konnte die Partei ab 2015 bei Landtagswahlen Erfolge verbuchen (Decker u. Best 2016), sodass sich die FDP-Gruppe entschied, hieran und insbesondere an das Plakatdesign der Berliner FDP zu den Abgeordnetenhauswahlen 2016 anzuschließen. ${ }^{22}$ Die diesem nachempfundene Hintergrund-Füllung mit stromlinienartigen, nach rechts oben zulaufenden Strahlen in den drei aktuellen Parteifarben soll Aufstieg und Zukunft suggerieren und der FDP einen progressiven Duktus verleihen. Auf dem von der FDP-Gruppe erstellten Personenplakat ist Christian Lindner, anders als der durch einen modernen Bildeffekt dezent kubistisch verfremdet anmutende Berliner Spitzenkandidat Sebastian Czaja in der Vorlage, als reale Person auf der von einem ruhigen Hintergrund-Blau dominierten, linken Plakathälfte unter dem neuen Parteilogo zu sehen. Die Gruppe entschied sich für die Personalisierungsstrategie, da Lindner das derzeit mit Abstand bekannteste

22 http://bit.ly/fdp-vorbild. Zugegriffen: 20.04.2017. 
Gesicht der öffentlich ansonsten nicht mehr so stark präsenten Partei darstelle und das neue, frische FDP-Image personifiziere. Durch das verwendete Bild ${ }^{23}$ soll Lindner sowohl seriös als auch locker wirken, um sowohl die alte Stammwählerschaft aus Selbstständigen und Mittelständlern wie auch ein junges Publikum anzusprechen. Die rechts auf drei ebenfalls in den drei Parteifarben gehaltenen Textkästen platzierte politische Botschaft besteht, erstens, aus dem Namen des Spitzenkandidaten, zweitens, dem auf die Klientel gemünzten Slogan „Globalisierung nutzen! Liberale Marktwirtschaft mit starkem Mittelstand!“ und, drittens, dem Claim „Die seriöse Alternative“ als Seitenhieb auf die AfD (und somit auch eine Nuance Negative Campaigning). Die FDP soll damit platziert werden als neue, frische Kraft für alle, die mit der Regierung unzufrieden sind, aber auch nicht AfD wählen wollen, bloß um ihren Protest zu bekunden. Außerdem inszenierte so die Gruppe die FDP als optimistische Kraft, die - entgegen dem Zeitgeist - in der Globalisierung mehr Chancen als Risiken sieht. Der über drei Zeilen verteilte Slogan erschien manchen Kursmitgliedern etwas zu lang geraten - eine Zweiteilung zur Generierung zweier ansonsten identischer Plakatvarianten wurde diskutiert. Im Sinne der Abkehr von dem Parteibeinamen „Die Liberalen“ erschien es manchen Teilnehmenden auch konsequenter, „freie“ an Stelle von „liberale Marktwirtschaft" zu titeln. Als weiteres Plakat entwarf die Gruppe noch ein reines Textplakat, das hier daher ebenfalls abgebildet werden kann (Abbildung 1). Als Hintergrund dient ein vergrößerter Ausschnitt der blau-gelb-magentafarbenen Stromlinien. Prominent in der Plakatmitte ist ein großer, gelber Textkasten platziert, auf dem in blauer Farbe „Update für Deutschland“ steht. Ergänzt wird dies durch vier ebenfalls gelb hinterlegte Hashtags ${ }^{24}$ in Magenta und einen QR$\operatorname{Code}^{25}$, die Modernität ausdrücken und die Brücke zum Medium Internet schlagen sollen. So sollen das 2013 als eindimensional wirtschafts- und finanzpolitisch wahrgenommene Profil der Partei verbreitert und das wiederum generalisiert für Modernität und Progressivität stehende Thema Digitalisierung besetzt werden. Das Plakat eignet sich zudem besonders zur Anziehung ehemaliger Wähler der Piraten-Partei, von deren früheren Vorsitzenden bereits zwei zur FDP gewechselt sind. Der Hashtag \#startupagain ist dabei mehrdeutig: Erstens soll die junge Gründerszene angesprochen werden, zweitens steckt hierin eine Anspielung auf eine Rede Lindners im nordrhein-westfälischen Landtag, in der dieser eine Kultur einforderte, die (unternehmerisches) Scheitern nicht hämisch kommentiert, sondern zweite Chancen zugesteht. Diese von Lindner durchaus charismatisch vorgetragene Rede verbreitete sich damals massiv über die sozialen Netzwerke. ${ }^{26}$ Drittens soll der Hashtag einen Schuss Selbstironie und Demut transportieren: Auch die neue FDP sei eine Art Startup-Unternehmen, sie sei 2013 gescheitert und bitte nun die Wähler um eine zweite Chance. Auch im Slogan „Update für Deutschland" kann man eine entsprechende zweite Ebene ausmachen: Deutschland brau-

23 http://bit.ly/fdp-lindner. Zugegriffen: 20.04.2017.

24 Hashtags werden vor allem auf Social Media-Plattformen wie Twitter und Facebook verwendet. Sie erleichtern die Auffindbarkeit von Schlagworten.

25 QR-Codes können von interessierten Passanten unkompliziert mit dem Smartphone eingescannt werden, woraufhin diese automatisch auf die Homepage der Partei weitergeleitet werden.

26 http://bit.ly/lindner-wutrede. Zugegriffen: 20.04.2017. 
che ein technisches Update in puncto Digitalisierung sowie ein politisches Update in Form der FDP, und die FDP habe auch gerade ein programmatisches Update hinter sich. Moniert wurde, dass der Parteiname insgesamt dreimal auf dem Plakat erscheint.

\section{Abbildung 1: Beispielplakate zu Bündnis 90/Die Grünen und FDP aus dem „Praxisseminar Wahlkampagne“27}
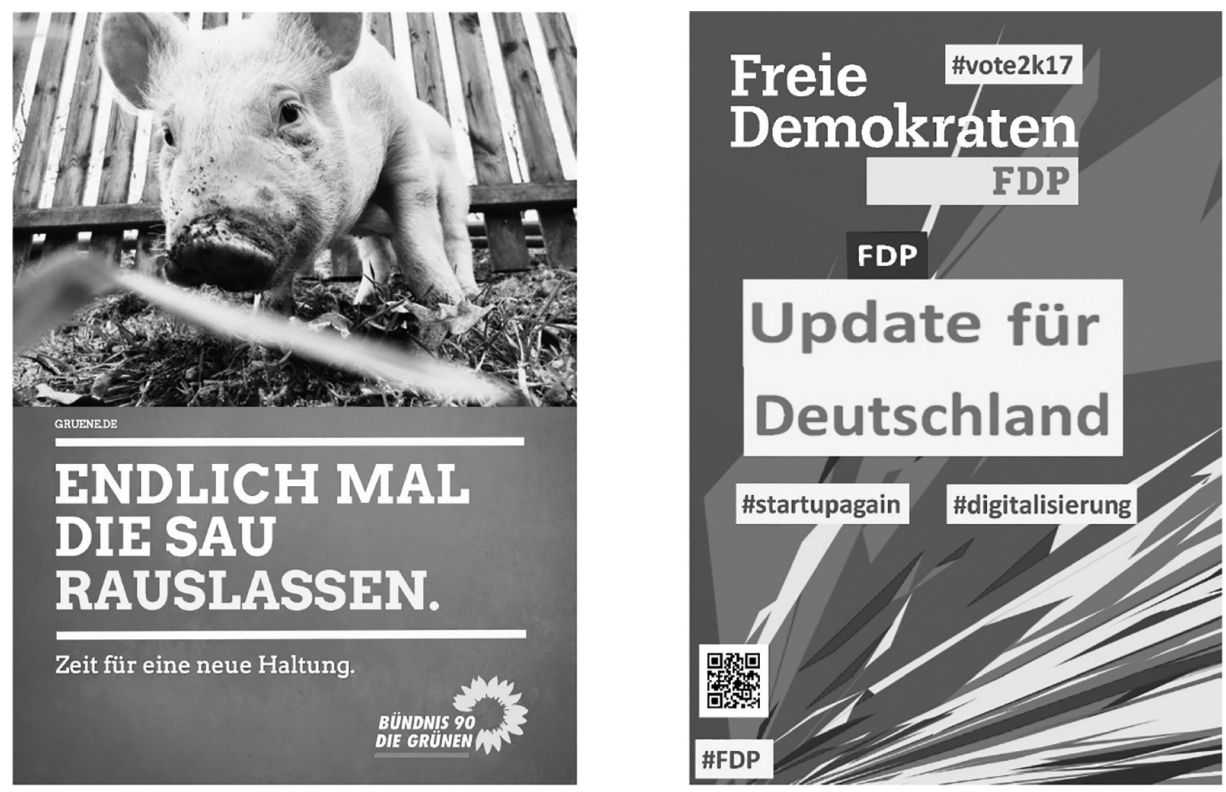

Die Linke-Gruppe beschloss, von den für die Partei typischen reinen Textplakaten abzuweichen, da mehrere Studien diese als wenig zugänglich ausweisen (Geise 2010, S. 80; Kercher 2011), und der Betrachterin oder dem Betrachter durch Piktogramme in der oberen Hälfte einen leichteren Einstieg ins Plakat zu ermöglichen. Da die Piktogramme von den Studierenden selbst geschaffen wurden, können die Plakatentwürfe hier abgebildet werden (Abbildung 2). Die inhaltliche Botschaft ist nicht - wie üblich - in Slogan und Claim organisiert, sondern besteht aus zwei im Prinzip gleichrangigen (wenn auch nicht in gleicher Schriftgröße gefassten) Text-Elementen, wovon der erste sehr allgemein gehalten und teilweise („Boni für alle“) mit einem ironischen Unterton versehen ist, während der zweite die konkrete Forderung beinhaltet (12 Euro Mindestlohn, Bau von Sozialwohnungen, 1050 Euro Mindestrente). Die beiden Textelemente sind mit weißen Balken hinterlegt und dynamisch in entgegengesetzter Richtung angeschrägt. Das Logo wird in gleicher Weise gesetzt und soll somit gewissermaßen zu einem dritten Element der Botschaft avancieren. Hierzu trägt auch die Übernahme des keilförmigen roten i-„,Punkts“ in den beiden anderen Textelementen bei. Das über-

27 Der Abdruck des im Grünen-Plakat zur Massentierhaltung verwendeten Bilds erfolgt mit freundlicher Genehmigung von Peta Deutschland e.V. 
greifende textliche Element der einzelnen Plakate ist das griffige „für alle“ im ersten Teil der Botschaft. In der Seminararbeit hob ein Gruppenmitglied hier die optimale Passung zum Titel des Wahlprogrammentwurfs „Sozial. Gerecht. Für alle. " hervor. Neben den konkret ausbuchstabierten Forderungen wird mit den genereller gehaltenen ersten Elementen der Botschaft auch noch auf weitere Themen angespielt, im Falle von „Boni für alle“ auf Managergehälter und generell Lohnungleichheit in Deutschland, im Falle von „Platz für alle“ auf das Flüchtlingsthema. Moniert wurde vereinzelt, dass keine eigene „für alle“-Formulierung zum Thema Mindestrente gefunden und die Forderung zum sozialen Wohnungsbau nicht genau so konkret gefasst wurde wie zu den beiden anderen Themen, so wie es mit „1 Million Sozialwohnungen“ möglich gewesen wäre. Die typische rote Hintergrund-Signalfarbe wurde beibehalten, aber durch eine hauchdünne weiße Rahmenlinie eingefasst. In Zusammenspiel mit den Piktogrammen (insbesondere der kämpferisch um den Geldschein geballten Faust) und der leicht schräg präsentierten Botschaft soll dies an Antifa-Aufkleber erinnern. Diese Assoziation sollte die Plakate der Linken von der „glatten“ Kommunikation der anderen Parteien abheben und das Milieu der Studierenden sowie prekär beschäftigten Akademiker als wichtiges Wählerreservoir der Partei in westlichen Städten ansprechen. Die Wohnungsbau-Szene und das Renten-Sparschwein muten eher niedlich als kämpferisch an und sollen Optimismus und Sympathie vermitteln, was früheren Plakaten der Linken nach Meinung der Gruppe eher fehlte und gerade in Zeiten einer ebenfalls vermehrt sozialpopulistisch auftretenden AfD die Linke positiv abheben könnte.

Abbildung 2: Beispielplakate zu Die Linke aus dem „Praxisseminar Wahlkampagne“
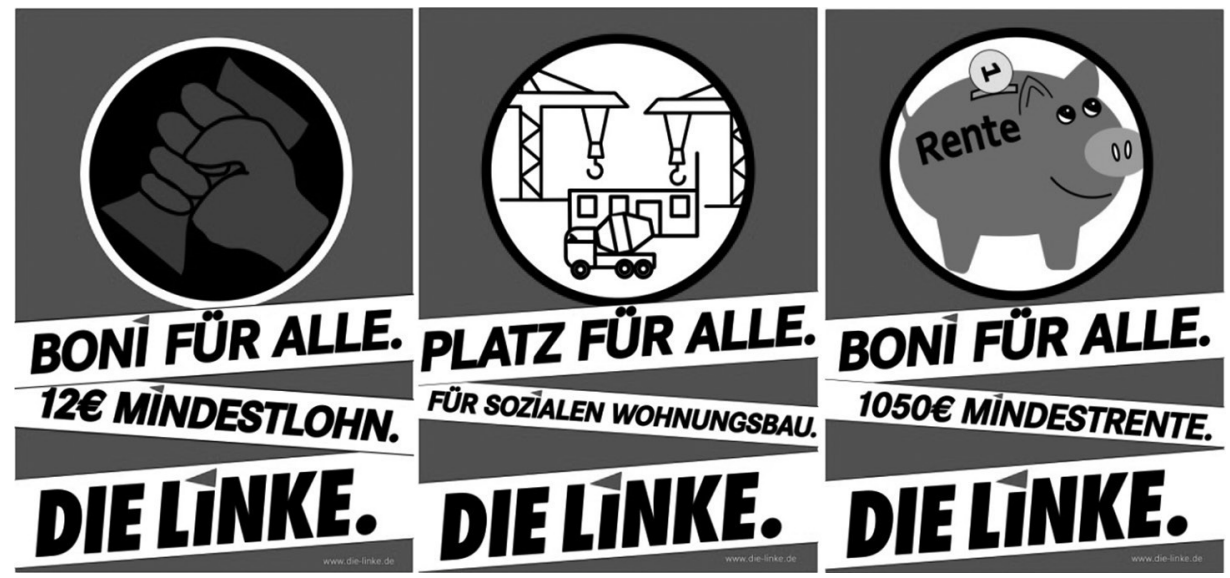

Die AfD-Gruppe produzierte ein Plakat, auf dem eine augenscheinlich misshandelte Frau abwehrend die Hand in Richtung der Betrachterin oder des Betrachters ausstreckt. ${ }^{28}$ Im Hintergrund ist der Kölner Dom nebst Silvesterfeuerwerk zu se-

28 http://bit.ly/afd-frau. Zugegriffen: 20.04.2017. 
hen $^{29}$ - eine unmissverständliche Anspielung auf die Vorkommnisse der Kölner Silvesternacht 2015. Das Feuerwerk ist AfD-bläulich eingefärbt. Oben auf dem Plakat prangt der Slogan „NICHT MIT UNS“. Das untere Drittel des Plakats wird von der AfD-blauen Wellenform ausgefüllt, worauf rechts das Parteilogo mit dynamischem, rotem Pfeil nach rechts oben sowie links ein „Störer“ organisiert sind, der mit einem stilisierten Kreuzchen propagiert: „WIR sind Deine Stimme!“. Die erst Mitte März vorgestellte AfD-Kampagne zur NRW-Landtagswahl enthielt übrigens ein Plakat, das in eine sehr ähnliche Richtung ging. Mit ihrem zweiten Bildplakat versuchte die Gruppe, den Begriff der Vielfalt zu Gunsten der AfD umzubesetzen. „Nur mit uns“ stand diesmal als Claim neben dem Logo im Bereich der blauen Welle im unteren Drittel. Das Bild darüber zeigte acht verschiedene Biergläser mit unterschiedlichen Biersorten darin. ${ }^{30}$ Der Slogan lautete „Deutsche Vielfalt erhalten“. In der Seminargruppe gingen die Meinungen zu diesem Versuch der Begriffsneubesetzung auseinander. Insbesondere wurde problematisiert, inwiefern denn eine Bedrohung deutscher Brauereivielfalt vorliege und ob die von den acht Biergläsern ausgelöste Stammtisch-Assoziation die Partei nicht eher in schädliche Nähe zur NPD rücken würde. Des Weiteren legte die AfD-Gruppe ein Textplakat vor, dessen Slogan „Mut zur Wahrheit. Einwanderung braucht Grenzen“ sich aber kaum von historischen Vorläufern wie „Mut zur Wahrheit. Einwanderung braucht klare Regeln“ und „Asyl braucht Grenzen“ unterscheidet.

\section{Feedback zum Seminar}

Das Feedback der Studierenden wurde einige Wochen vor Semesterende per OneMinute-Paper eingeholt, sowie kurz darauf dann auch im Rahmen der am Institut für Politische Wissenschaft und Soziologie regelmäßig im Paper-Pencil-Verfahren durchgeführten standardisierten Lehrveranstaltungsevaluation, an der sich 25 Kursmitglieder beteiligten. Nicht weniger als 92 Prozent der Befragten gaben an, das Seminar (u. a.) aus starkem Interesse am Thema gewählt zu haben. Üblicher sind hier Werte zwischen 50 und 75 Prozent. Andere Beleggründe wurden nur vereinzelt genannt. ${ }^{31}$ In der Gesamtbewertung des Seminars vergaben 52 Prozent die Note „sehr gut“, 36 Prozent die Note „gut“ und 12 Prozent die Note „befriedigend".

92 (52/40) Prozent ${ }^{32}$ beurteilten die Gestaltung der Veranstaltung als abwechslungsreich. Im freien Kommentarteil der Evaluation und in den One-MinutePapers wurde das Seminarkonzept als innovativ, aktualitäts- und praxisbezogen gelobt. So schrieben einige Teilnehmende, man habe „Politik selber gestalten und

29 http://bit.ly/afd-dom. Zugegriffen: 20.04.2017.

30 http://bit.ly/afd-bier. Zugegriffen: 20.04.2017.

31 Der Dozent wurde als Grund nur von 16 Prozent (also vier Studierenden) genannt; Veranstaltungszeitpunkt, Zuteilung durch die Belegungssoftware, kleinstes Übel unter den angebotenen Seminaren und Sonstiges von je 4 Prozent (also einem einzelnen Studierenden).

32 Die standardisierte Evaluation arbeitet überwiegend mit fünfstufigen Skalen, mit denen die Zustimmung zu Aussagesätzen von „stimme voll und ganz zu“ (1) bis „stimme überhaupt nicht zu“ (5) angegeben werden kann, wobei nur diese beiden Skalenendstufen benannt sind. Der Einfachheit wegen werden im Folgenden die Skalenstufen 1 und 2 zusammengerechnet und in Klammern die Einzelwerte für Stufe 1 bzw. 2 genannt. Die Fragen wurden von 21 bis 25 Befragten beantwortet. 
anwenden [können], statt sich nur mit Theorien zu beschäftigen“, „tolle praktische Tätigkeiten angewandt, endlich mal praktische Politik betrieben, nah an der Realität“ und einen „Bezug zu einem möglichen Berufsfeld für Politikwissenschaftler" erhalten. Alle Befragten beurteilten die Studienleistung im Kontext des Seminars als nützlich. 92 (50/42) Prozent gaben an, Präsentationskompetenz erworben zu haben, 71 (29/42) Prozent bestätigten, Kompetenzen im Bereich der Debattenkultur, und 57 (14/43) Prozent, Kompetenzen in der Entwicklung wissenschaftlicher Fragestellungen entwickelt zu haben. Neben Kompetenz konnten die Studierenden auch Autonomie erleben: „Praxisanteile waren eine gute Erfahrung und haben zum ,Selber-Denken“ angeregt", „das Seminarthema fördert eigenverantwortliches Arbeiten", und „eigene Arbeit wurde ermöglicht". Einige forderten das Angebot weiterer Praxisseminare im Politikwissenschaftsstudium. 77 (41/36) Prozent bekundeten, die Veranstaltung habe thematisch gut in ihren Studienverlauf gepasst. Eine Teilnehmerin meldete sogar rück: „In diesem Seminar habe ich die Bestätigung erhalten, mein Masterstudium in Politikwissenschaft weiterzuführen."

Auch die Interaktivität wurde gelobt. Aus Sicht von 92 (72/20) Prozent gab es genug Raum für Diskussionen, 75 (25/50) Prozent gaben an, sich selbst häufig mit eigenen Beiträgen beteiligt zu haben, 83 (38/46) Prozent bescheinigten der Mehrzahl der anderen Kursmitglieder eine engagierte Beteiligung. In den Worten eines Studierenden: „Sehr interaktiv, die Zeit geht damit sehr schnell rum. Tolle Diskussionen. " Ein anderer unterfütterte den Motivationsfaktor soziale Eingebundenheit: „Arbeit in Projektgruppen ermuntert zur regen, kontinuierlichen und aktiven Teilnahme“. Die Anwesenheitsquote war durchgängig sehr hoch. Trotz im Land Nordrhein-Westfalen nicht mehr bestehender Anwesenheitspflicht gaben bei der Evaluation in der drittletzten Semesterwoche 88 Prozent der Teilnehmenden an, bis dahin höchstens zwei Mal gefehlt zu haben. Die übrigen 12 Prozent beschränkten sich auf drei oder vier Abwesenheiten. Zu den Gründen des Fernbleibens gab nur ein Studierender mangelndes Interesse am Seminar an. Von ursprünglich 33 Teilnehmenden brach auch nur eine einzige Studierende das Seminar vorzeitig ab (aus persönlichen Gründen).

Dass die Aufgabenstellung mit der Gestaltung der Plakate ein kreatives Element aufwies, wurde positiv hervorgehoben: „konnte kreativ werden, eigene Ideen einbringen \& umsetzen “, „gut: selbstständiges, kreatives Arbeiten wird gefördert", „[k]reative Arbeiten sind normalerweise kaum vorhanden in Politik-/ Sozialwissenschaften, deswegen gute Abwechslung ". Es wurde aber auch deutlich, dass der kreativen Anwendung theoretische Kenntnisse zu Grunde gelegt werden mussten: „Wir dürfen kreativ sein, was mir sehr gefällt, müssen aber theoretisches Wissen auch haben. " Der Transfer des theoretischen Wissens in die Praxis wurde als gelungen empfunden: „[S]uper Konzept, das Theorie und Praxis gekonnt verbindet und neben strategischem Denken auch Kreativität erfordert hat".

Die Lernatmosphäre wurde von 87 (48/39) Prozent als angenehm beurteilt. Selbst Studierende, deren Studienschwerpunkt das Thema nicht entsprach, attestierten der Veranstaltung einen Spaßfaktor: „So machen Seminare auch Spaß!“ Zugleich bestand der Eindruck eines effektiven Lernens: „lockere Arbeitsatmosphäre, aber trotzdem intensive Arbeit". Hierbei war die aktive Rolle der Kursmitglieder entscheidend: „lernintensiv, da aktive Auseinandersetzung mit jeweili- 
ger Partei!" Angesichts der Veranstaltungszeit 16-18 Uhr befand ein Studierender: „Trotz langem Unitag konnte man gut folgen, da die Themen sebr praxisnah waren". Auch von den Ergebnissen der anderen Gruppen konnten die Studierenden profitieren: „Auswertung sebr interessant: wenn man auch die Ergebnisse der anderen Gruppen sieht" und „größeres Verständnis für ideologisch ferne Parteien“. Alle Befragten bescheinigten der Veranstaltung einen grundlegenden Überblick über das Thema und 92 (40/52) Prozent bestätigten die Vermittlung vertiefender Kenntnisse. 72 (32/40) Prozent stimmten der Aussage zu, sie hätten in der Veranstaltung viel gelernt. Der Anspruch des Seminars war aus Sicht von 74 Prozent genau richtig, 22 Prozent fühlten sich etwas unterfordert, und 4 Prozent etwas überfordert. 91 Prozent beurteilten die Stoffmenge als genau richtig, wohingegen sich 9 Prozent mehr Lerninhalte gewünscht hätten. Auf nachhaltiges Lernen deutet zumindest das folgende Zitat hin: „Mi[ch] hat das Seminar [...] motiviert, mich mit meiner Partei intensiver auseinanderzusetzen. "

Auch die Erstellung von Infopostern in gemischten Gruppen und deren Vorstellung in Puzzlegruppen im Theorieteil wurde zumeist positiv beurteilt: „anregende didaktische Methode in Gruppenarbeit u. Diskurs erlernte Inhalte zu systematisieren und festzuhalten". Allerdings wurde die dabei in dem zu kleinen Raum entstehende Lautstärke mitunter moniert. Der Aussage, die begleitenden Texte seien hilfreich gewesen, stimmten 86 Prozent der Befragten zu. 96 (76/20) Prozent bestätigten, dass in der Veranstaltung Bezug auf die begleitenden Materialien genommen wurde: „Texte zur Vorbereitung auf die Sitzungen sind angemessen, nicht zu viel; und toll aufgeteilt unter den Teilnehmern. Somit konnten wir effizient gemeinsam an den Texten arbeiten. "Auch, dass die zu lesenden Texte den Gruppen/Personen einzeln zugeteilt wurden, war eine clevere Strategie. So waren die Leute in der Regel vorbereitet."

Der Arbeitsaufwand für die Studierenden wurde als hoch eingeschätzt. Besonders durch die unterschiedlichen Fähigkeiten in Bezug auf Grafikprogramme ergaben sich Unwuchten in der gruppeninternen Arbeitsverteilung: „[S]eitdem es um die Erstellung der Plakate geht, hat sich mein Workload potenziert und der meiner Kollegen auf Kommentieren und Verbesserungsvorschläge geben beschränkt". Die zur Vor- und Nachbereitung der Veranstaltung wöchentlich benötigte Zeit ${ }^{33}$ schätzten 56 Prozent auf ein bis zwei Stunden und 16 Prozent auf zwei bis drei Stunden. Zwei Studierende gaben mehr als fünf Stunden an. Allerdings wählten zu der Aussage „Den zeitlichen Aufwand für das Selbststudium kann ich insgesamt bewältigen“ 65 Prozent die „trifft zu“- und 35 Prozent die zweite Kategorie auf einer vierstufigen Skala.

Trotz diverser Änderungen des Zeitplans im Laufe des Semesters attestierten 92 (52/40) Prozent der Lehrveranstaltung ein gut durchdachtes Konzept. Vereinzelte Kritik gab es in puncto Zeitmanagement, wobei manche „zu viel Vorlauf mit Textarbeit“ monierten, während andere „zu viel Leerlauf in der Plakaterarbei-

33 In der Fragestellung wird darum gebeten, die für die eigene Studienleistung benötigte Zeit hierbei nicht einzurechnen. Inwiefern diesem Hinweis nachgekommen wird - insbesondere, wenn die Studienleistung nicht wie bei einem Referat punktuell erfolgt, sondern sich nahezu über das ganze Semester erstreckt -, ist allerdings fraglich. 
tung“ ausmachten. Die auseinanderlaufenden Beurteilungen des Zeitmanagements sind wohl auf die individuellen Gruppenerfahrungen in Verbindung mit der vom Dozenten vage gelassenen Erwartungshaltung hinsichtlich der Zahl der zu produzierenden Plakate zurückzuführen. Ermüdungserscheinungen scheinen tendenziell bei den Gruppen aufgetreten zu sein, die es - aufgrund geringem grafischen Selbstvertrauen oder kritischem gruppeninternen Arbeitsungleichgewicht beim geringfügigen Überarbeiten der ersten zwei oder drei Plakate beließen, während andere Gruppen bis zur wortwörtlich letzten Minute neue Plakate produzierten. Einzelne Teilnehmende konnten die im Seminar für die Plakaterstellung freigeräumte Zeit „aufgrund technischer Ausstattung “ nicht optimal nutzen: „Adobe-Arbeit im Seminar war nicht möglich, lediglich Konzept + Besprechung. " Eine Rolle dürfte auch das frühzeitige Einfordern erster Plakate durch den Dozenten mit Blick auf die Einladung der Praxis-Experten gespielt haben.

\section{Fazit und Ausblick}

Insgesamt ist zu dem „Praxisseminar Wahlkampagne“ ein sehr positives Fazit zu ziehen. Hierzu wird zunächst noch einmal auf die in Kapitel 2 geschilderten Lehrbzw. Lernziele eingegangen.

Die affektiven Lernziele konnten weitgehend realisiert werden. Die Motivation der Studierenden lag in dieser Veranstaltung dank Kompetenzerleben, Autonomieerleben, sozialer Einbindung und der kreativen Aufgabenstellung deutlich über dem üblichen Maß, was sich auch in einer beispiellosen Anwesenheit niederschlug. Die Vorbereitungslektüren im ersten Seminarteil wurden tatsächlich von so gut wie allen gelesen. In den meisten Gruppen herrschte ein gutes Arbeitsklima. Die kreative Aufgabenstellung und die unkonventionelle Lehr-Lernsituation wurden positiv bewertet und dem Bundestagswahlkampf 2017 wurde eine besondere Aufmerksamkeit zuteil. Freilich wäre bezüglich der affektiven Zielerreichung, insbesondere was die Motivation anbelangt, eine umfassendere empirische Begleitforschung wünschenswert.

Die kognitiven Lernziele wurden ebenfalls in einem hohen Maße erreicht. Dies zeigen, erstens, die - auch seitens der Parteiakteure so eingeschätzten - guten bis sehr guten Kampagnenentwürfe, mit denen die Gruppen die Theorie in die Praxis umsetzten, zweitens, das erfreuliche Niveau der eingereichten Seminararbeiten und, drittens, die fachkundigen Diskussionen und Verbesserungsvorschläge zu den Entwürfen der anderen Gruppen. Ob sich das skizzierte besonders nachhaltige Lernen mit Lerneffekten noch ein halbes Jahr nach Seminarende tatsächlich realisieren lässt, wäre eine spannende Frage für die empirische Lehr-/Lernforschung, die hier leider nicht beantwortet werden kann.

Die arbeitstechnischen Lernziele konnten nur teilweise bewerkstelligt werden. Die Erstellung und Vorstellung der Infoposter gelang meist gut. Auch präsentierten die Studierenden ihre Plakatentwürfe nicht nur vor der Gruppe, sondern auch in der eher ungewohnten Situation vor den Parteipraktikern selbstbewusst und kompetent, und zeigten sich im Stande, Feedback zu geben und anzunehmen. Teilweise mangelte es allerdings an der Fähigkeit der Gruppen zur Selbstorganisation zwischen den Sitzungen, wodurch die Studienleistung in größerem Maße, als ur- 
sprünglich vorgesehen, in die Seminarsitzungen verlagert werden musste. Eine 14-tägige Sitzungsfrequenz oder eine Durchführung in mehreren Blöcken in längeren zeitlichen Intervallen hätte dieses Problem wahrscheinlich eingedämmt. Dies schlugen auch einige Studierende in der Evaluation vor.

Die Nutzung des Wikis fiel den Teilnehmenden unerwartet schwer, und nur eine Gruppe bediente sich dieses Instruments weiter, um neue Erkenntnisse (etwa aus den Infopostern) im Nachgang der initialen Situationsanalyse festzuhalten. Bei einer nochmaligen Durchführung könnte es eine gute Idee sein, eine durchgängige Nutzung des Wikis verbindlich einzufordern und die Studierenden damit auch im Sinne eines zusätzlichen arbeitstechnischen Lernziels auf in der Arbeitswelt zunehmend verbreitete neue Arbeitsformen (Digitalisierung) vorzubereiten.

In Bezug auf den Umgang mit Grafikprogrammen setzten die meisten Gruppen auf die Expertise einzelner Studierender, für die sich damit eine deutlich höhere Arbeitsbelastung ergab. Anscheinend gelang es nur unzureichend, die Aufgabenstellung als Chance herauszustellen, sich mit der Bedienung von Grafikprogrammen vertraut zu machen und dabei von den grafisch Fähigeren in der Gruppe zu lernen. Dies dürfte auch damit zusammenhängen, dass die grafisch Fähigeren unter Umständen besonders komplexe und/oder teure Programme bevorzugen, was den grafisch noch wenig Beschlagenen die Anreize zum Peer-Learning nimmt. Ideal wäre eine kurze Einführung aller Studierenden in ein nicht allzu komplexes und günstig anzuschaffendes Programm oder sogar Freeware-Programm wie GIMP oder Scribus. Das „Praxisseminar Wahlkampagne“ selbst bietet - jedenfalls als Standardlehrveranstaltung von zwei Semesterwochenstunden - hierfür aber wohl keine ausreichenden Zeitressourcen. Sofern ein solcher Input nicht etwa mittels Ressourcen des Hochschulrechenzentrums ausgelagert werden kann, sollten die grafisch Fähigeren zumindest dadurch entlastet werden, dass den Gruppen nahegelegt wird, ihnen ebenfalls zeitaufwändige, für die Plakaterstellung erforderliche Teilaufgaben wie etwa das Suchen nach brauchbarem Bildmaterial im Internet komplett abzunehmen.

Sofern hingegen eine bessere gruppeninterne Verteilung des Arbeitspensums gewährleistet werden kann, können einer Gruppe durchaus drei bis vier Plakate verbindlich abgefordert werden. Eine Vorgabe von mindestens einem Plakat pro Gruppenmitglied könnte ebenfalls in Erwägung gezogen werden. Diese wären dann nach wie vor von der Gruppe gemeinsam zu konzipieren, dann aber tatsächlich arbeitsteilig umzusetzen, wobei das erste Plakat von einem grafisch beschlagenen Gruppenmitglied im Sinne des Peer-Learning vor den Augen der anderen realisiert werden sollte. Dabei könnte der inhaltliche Fokus noch verbreitert werden, indem für eins der Plakate die Vorgabe „Negative Campaigning“ getroffen wird. Auch könnten die Gruppen stärker in Beziehung zueinander gesetzt werden, wenn mit dem letzten realisierten Motiv ein bereits von einer anderen Gruppe vorgelegtes Plakat beantwortet werden müsste, wie es im vorliegenden Fall die GrünenGruppe mit dem CSU-Gastfreundschafts-Plakat getan hat. Ein Personenplakat könnte ebenfalls eingefordert werden, falls dadurch der Themenwahlkampf nicht zu stark verengt wird.

Erwägenswert wäre weiterhin, die Strategieentwicklung zu einem eigenen Teilschritt aufzuwerten und die Theorie-Phase so auseinanderzuziehen. Auf die Situa- 
tionsanalyse (im engeren Sinne) folgten Texte zu Wahlkampfstrategien, woraufhin Strategie und Gewinnerthemen entwickelt werden müssten, dann erst gäbe es Input zum Thema Plakatgestaltung.

Das Interesse der Praxisakteure an den Strategie- und Plakatideen von Studierenden scheint ausgeprägt zu sein. Außer von der die Politikwissenschaft generell eher skeptisch beäugenden AfD und der CSU, für die eine Anreise nach Bonn einen unvergleichlich höheren Aufwand bedeutet hätte, kamen von allen Parteien Politiker oder Praktiker zum Seminarbesuch. Das Feedback zu den CSU-Plakaten konnte dankenswerterweise von dem CDU-Praxisexperten übernommen werden. Ein Politiker, der aus privaten Gründen für den vorgesehenen Termin absagen musste, drang sogar seinerseits auf einen Ausweichtermin.

Studierende so voller Elan „bei der Sache“ zu sehen, wirkt auch auf den Dozenten motivierend. Gewöhnungsbedürftig ist allerdings die Zurückhaltung, die man sich in einem solchen Lehr-Lern-Kontext auferlegen muss. Insbesondere in den Sitzungen, die dem Fortkommen der Gruppenarbeit gewidmet waren, sollten die Gruppen tatsächlich erst einmal eine halbe Stunde sich selbst überlassen werden und erst danach zwecks Kontrolle des Fortkommens, Feedback und ggf. Anregungen aufgesucht werden. Der Arbeitsaufwand des Dozenten ist insgesamt nicht unbedingt höher als bei einem Referate-Seminar, er verteilt sich aber unregelmäßiger. Der Input-Teil zu Beginn muss sehr gut vorbereitet sein, die Vorbereitungstexte müssen gründlich ausgewählt und sorgsam unter den Studierenden aufgeteilt werden. Insbesondere bei den für die Situationsanalyse zu verwendenden Texten ist auf einen hohen Aktualitätsgrad zu achten. Im vorliegenden Fall galt dies insbesondere mit Blick auf die Flüchtlingskrise und die Entwicklung der AfD. Die Einladung und Koordination der Praktiker ist auch nicht zu unterschätzen. Während die Gruppenarbeitsphase läuft, hat der Dozent eher weniger zu tun. Weil er trotzdem präsent und ansprechbar sein muss, ergibt sich daraus allerdings keine wirkliche Zeitersparnis in der Durchführung.

Ob das „Praxisseminar Wahlkampagne“ als regelmäßige Lehrveranstaltung funktionieren würde, erscheint fraglich, da es von dem Aktualitätsbezug zu einer zukünftigen Wahl lebt. Als Gegenstand dürften allerdings auch Landtags- und Europawahlen ebenso wie ggf. Volksentscheide geeignet sein, sodass man auch nicht unbedingt vier Jahre bis zur nächsten Bundestagswahl warten muss. Auch Kommunalwahlen könnten genutzt werden, sofern diese auf ein hinreichendes Grundinteresse der Studierenden stoßen. Immerhin stünden hier die Chancen besonders gut, dass die eigenen Plakatentwürfe tatsächlich zur Wahlkampfzeit im Stadtbild auftauchen. Im Falle der oben abgebildeten Plakate der LinksparteiGruppe scheint der Abgeordnete diese tatsächlich in seinem Wahlkreis aufhängen zu wollen; er hat sogar bei einem Mitglied der Gruppe ein weiteres Plakat zum Thema fahrscheinlose Nutzung des Öffentlichen Personennahverkehrs in Auftrag gegeben. Ansonsten wird es nicht nur für die Studierenden interessant sein, die (zum Zeitpunkt der Abfassung dieses Artikels Ende April 2017 noch nicht veröffentlichten) tatsächlichen Plakatkampagnen der Parteien mit den hier vorgestellten zu vergleichen. Jetzt schon sicher erscheint, dass Parteien vom Input von Studierenden der Politikwissenschaft durchaus profitieren können. 


\section{Literatur}

Best, Volker. 2015. Komplexe Koalitionen, perplexe Wähler, perforierte Parteiprofile. Eine kritische Revision jüngerer Befunde zur deutschen Koalitionsdemokratie und ein Reformvorschlag. Zeitschrift für Parlamentsfragen 46: 82-99. DOI: 10.5771/0340-17582015-1-82.

Bloom, Benjamin S., Max D. Engelhart, Edward J. Furst, Walker H. Hill und David R. Krathwohl. 1973. Taxonomie von Lernzielen im kognitiven Bereich. Weinheim/Basel: Beltz Verlag.

Deci, Edward L., und Richard M. Ryan. 1993. Die Selbstbestimmungstheorie der Motivation und ihre Bedeutung für die Pädagogik. Zeitschrift für Pädagogik 39: 223-238.

Decker, Frank, und Volker Best. 2016. Wiederaufstieg oder endgültiger Abstieg? Die FDP zur Halbzeit der Auszeit. Gesellschaft - Wirtschaft - Politik 65: 43-52.

Geise, Stephanie, und Frank Brettschneider. 2010. Die Wahrnehmung und Bewertung von Wahlplakaten. Ergebnisse einer Eyetracking-Studie. In Information - Wahrnehmung - Emotion. Politische Psychologie in der Wahl- und Einstellungsforschung, Hrsg. Thorsten Faas, Kai Arzheimer und Sigrid Roßteutscher, 71-96. Wiesbaden: VS Verlag für Sozialwissenschaften. DOI: 10.1007/978-3-531-92336-9_5.

Gruber, Hans. 2007. Gruppenarbeit. In Förderung von Kompetenzen in der Hochschullehre. Theoretische Konzepte und ihre Implementation in der Praxis, Hrsg. Birgit Hawelka, Marianne Hammerl und Hans Gruber, 59-70, Kröning: Asanger Verlag.

Holtz-Bacha, Christina, und Eva-Maria Lessinger. 2006. Politische Farbenlehre. Plakatwahlkampf 2005. In Die Massenmedien im Wahlkampf. Das Wahljahr 2009, Hrsg. Christina Holtz-Bacha, 117-139. Wiesbaden: VS Verlag für Sozialwissenschaften. DOI: 10.1007/978-3-531-90383_3.

Johansen, Kathrin, Karsten Jung, Susanne Lexa und Yvonne Niekrenz. 2010. Einsteigerhandbuch Hochschullehre. Aus der Praxis für die Praxis. Darmstadt: WBG.

Kercher, Jan. 2011. Ein Plakat sagt mehr als 1000 Worte? Die Plakat-Kampagnen zur Landtagswahl 2011 in Baden-Württemberg im Vergleichstest. Eine Studie der Universität Hohenheim. https://komm.uni-hohenheim.de/uploads/media/Plakat-Studie_LTW_ BW_2011.pdf. Zugegriffen: 20.09.2016.

Leideker, Melanie. 2010. Angreifende Plakatwerbung im Wahlkampf - effektiv oder riskant? Ein Experiment aus Anlass der SPD-Europawahlplakate 2009. In Die Massenmedien im Wablkampf. Das Wabljahr 2009, Hrsg. Christina Holtz-Bacha, 117-139. Wiesbaden: VS Verlag für Sozialwissenschaften. DOI: 10.1007/978-3-531-92509-7_5.

Lessinger, Eva-Maria, und Christina Holtz-Bacha. 2010. „Wir haben mehr zu bieten“. Die Plakatkampagnen zur Europa- und Bundestagswahl. In Die Massenmedien im Wahlkampf. Das Wahljahr 2009, Hrsg. Christina Holtz-Bacha, 67-116. Wiesbaden: VS Verlag für Sozialwissenschaften.

Lessinger, Eva-Maria, Christina Holtz-Bacha und Willi Cornel. 2015. Wahlplakate treffen jeden. Die Plakatkampagnen der Parteien im Bundestagswahlkampf 2013. In Die Massenmedien im Wahlkampf. Die Bundestagswahl 2013, Hrsg. Christina Holtz-Bacha, 91-120. Wiesbaden: VS Verlag für Sozialwissenschaften.

Müller, Florian H. 2007. Studierende motivieren. In Förderung von Kompetenzen in der Hochschullehre. Theoretische Konzepte und ihre Implementation in der Praxis, Hrsg. Birgit Hawelka, Marianne Hammerl und Hans Gruber, 31-43, Kröning: Asanger Verlag. 
Müller, Marion G. 1997. Politische Bildstrategien im amerikanischen Präsidentschaftswahlkampf 1828-1996. Berlin: Akademie-Verlag.

Schoofs, Jan, und Jan Treibel. 2014. Depolarisierung und Multioptionalität? Wettbewerbsstrategien der Parteien in den Bundestagswahlkämpfen 2009 und 2013. In Bilanz der Bundestagswabl 2013. Voraussetzungen, Ergebnisse, Folgen, Hrsg. Eckhard Jesse, und Roland Sturm, 57-74, Baden-Baden: Nomos. DOI: 10.5771/9783845252513-57.

Strøm, Kaare. 1990. A Behavioral Theory of Competitive Political Parties. American Journal of Political Science 34: 535-598. DOI: 10.2307/2111461.

\section{Autorenangaben}

Dr. Volker Best

Institut für Politische Wissenschaft und Soziologie

Rheinische Friedrich-Wilhelms-Universität Bonn

Lennéstraße 27

53113 Bonn

E-Mail: vbest@uni-bonn.de 


\title{
ZUSAMMENFASSUNGEN
}

\author{
Sabrina Jasmin Mayer/Martin Schultze \\ Multiple Parteiidentifikation, politische Involvierung und Cross-Pressures
}

In diesem Beitrag untersuchen wir Mehrfachparteibindungen in Deutschland. Auf Basis einer bevölkerungsrepräsentativen Umfrage und mithilfe eines neuen Messinstruments zur Erfassung multipler Bindungen können wir zeigen, dass Mehrfachparteibindungen im deutschen Elektorat in nennenswerter Weise vorhanden sind: Knapp 30 Prozent der Befragten besitzen dabei eine solche Mehrfachparteibindung. Politisches Interesse und Bildung als Facetten der politischen Involvierung begünstigen dabei die Herausbildung von Mehrfachparteibindungen innerhalb eines politischen Lagers. Die Zugehörigkeit zu konfligierenden Großgruppen hat hingegen keinen signifikanten Effekt auf die Herausbildung multipler Parteiidentifikationen.

\section{Thomas Gschwend/Helmut Norpoth}

Machtwechsel in Sicht? Die Vorhersage des Kanzlermodells für die Bundestagswahl 2017

Das Kanzlermodell sagt voraus, dass bei der Bundestagswahl 2017 höchstwahrscheinlich Angela Merkel für eine weitere Amtszeit als Regierungschefin wiedergewählt wird. Stand Juni dieses Jahres führt sie so deutlich gegenüber ihrem sozialdemokratischen Herausforderer Martin Schulz in der Kanzler-Popularität, um jedweden Abnützungseffekt nach ihren nun drei Amtszeiten mehr als wett zu machen. Neben der Fortsetzung der Großen Koalition hat Merkel auch die Möglichkeit, eine Jamaika-Koalition (mit der FDP und den Grünen) zu bilden, die im neuen Bundestag eine Mehrheit haben wird. Eine Veränderung der Popularitätswerte würde die Prognose jedoch ändern. Für den Fall, dass Schulz letztlich Merkel in der Kanzler-Popularität übertrifft, wie er es Ende Februar getan hat, würde eher der SPD-Kandidat die nächste Bundesregierung als Kanzler führen.

\section{Arndt Leininger/Mark Kayser \\ Eine länderbasierte Prognose zur Bundestagswahl 2017}

Wenn der Wahltermin noch in weiter Ferne liegt, stellen Umfragen keine guten Prognosen dar. Zu wenige Wähler interessieren sich zu einem so frühen Zeitpunkt schon für die Wahl und zu viele Umstände können sich bis dahin noch ändern. Zwar eignen sich sogenannte strukturelle Modelle dazu, grundlegende Erwartungswerte aufzustellen, jedoch sind diese aufgrund kleiner Stichproben sehr unsicher und ungenau. Um diesen Mängeln zu begegnen, beruht unsere frühzeitige Prognose der Bundestagswahlergebnisse 2017 sowohl auf politischen und wirtschaftlichen Daten als auch auf Resultaten vorangegangener Landtagswahlen. Ein Mehrebenenmodell liefert dabei die Schätzwerte. Landtagswahlen finden zu unterschiedlichen Terminen statt und bringen den Vorzug mit sich, nicht nur tatsächliche Wählerpräferenzen, sondern auch neue politische Themen zu erfassen. Wir argumentieren, dass dieser Ansatz deshalb eine vielversprechende Methode für frühzeitige Prognosen darstellt, wenn Umfragen noch nicht aussagekräftig sind. 


\section{Simon Munzert/Lukas Stötzer/Thomas Gschwend/Marcel Neunhoeffer/Sebastian Sternberg} Zweitstimme.org. Ein strukturell-dynamisches Vorhersagemodell für Bundestagswahlen

Wir berichten die Ergebnisse einer ex-ante-Vorhersage der Zweitstimmenergebnisse für die Bundestagswahl 2017. Dazu kombinieren wir Daten veröffentlichter Umfragen mit strukturellen Informationen. Das Modell trägt den Eigenheiten von Vielparteiensystemen Rechnung und erlaubt es, Aussagen über die Wahrscheinlichkeit bestimmter Wahlergebnisse zu treffen, z. B. der Mehrheit der Sitzanteile für eine Partei oder der rechnerischen Mehrheit für verschiedene Koalitionsoptionen. Die Vorhersagen des Modells werden auf der Plattform zweitstimme.org veröffentlicht und aktualisiert. Unser Ansatz hat dabei nicht nur akademischen Wert: Wir geben Journalisten, Experten und Bürgern Informationen an die Hand, die helfen können, tatsächliche Parteiunterstützung einzuschätzen und letztlich besser informierte Wahlentscheidungen zu treffen.

\section{Oliver Strijbis}

\section{Nicht was Du denkst! Was wir wirklich von Wahlvorhersagen lernen können}

Wie dieses Forum zeigt, wird der Wettbewerb von Vorhersagemodellen nach US Vorbild auch in Deutschland zur gängigen Praxis. Dabei stellt sich die Frage nach dem wissenschaftlichen Sinn dieses Unterfangens. Ein oft hervorgebrachtes Argument ist, dass Wahlvorhersagen, indem sie vor der Wahl gemacht werden, einen besonders harten und daher guten Test für Wahltheorien darstellen. In diesem Beitrag bestreite ich dieses Argument und zeige, dass die in diesem Heft vorgestellten Vorhersagemodelle kaum zur Falsifizierung von Theorien beitragen. Dagegen verweise ich auf eine weitgehend übersehene Relevanz von Wahlvorhersagen, welche in ihrem Potenzial zur Operationalisierung wichtiger Variablen besteht. Ich illustriere dieses Argument mit Bezug auf die Literatur zu Prognosemärkten.

\section{Volker Best}

Studierende im Wahl-Werber-Fieber. Die Erstellung von Wahlplakaten zur Bundestagswahl 2017 im „Praxisseminar Wahlkampagne“

Der Beitrag stellt das Lehr-Lern-Konzept eines „Praxisseminar Wahlkampagne“ vor, in dessen Rahmen Studierendengruppen eine Wahlkampfstrategie jeweils einer Partei entwerfen und kreativ in eine Plakatkampagne umsetzen. Das Konzept wurde im Vorfeld der Bundestagswahl 2017 mit Master-Studierenden erprobt. Dabei konnten die kognitiven und affektiven Lehr-/Lernziele weitgehend erreicht werden. Insbesondere wirkten sich das Erleben eigener Kompetenz und Autonomie sowie sozialer Einbindung sehr positiv auf die Motivation der Studierenden aus. Hinsichtlich der arbeitstechnischen Lernziele, die nur teilweise realisiert werden konnten, werden Ideen zur Verbesserung des Konzepts erörtert. 